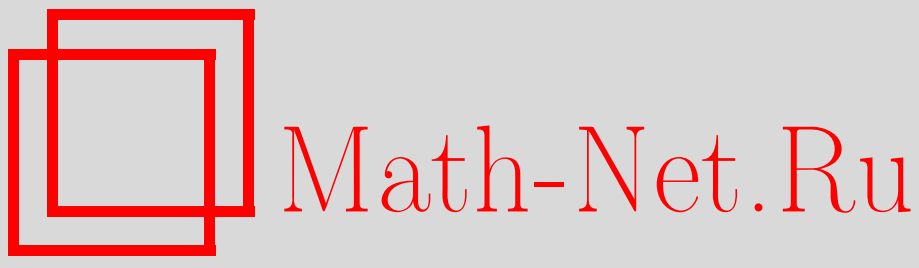

С. Г. Танкеев, O весах $l$-адического представления и арифметике собственных чисел Фробениуса, Изв. РАН. Сер. матем., 1999, том 63, выпуск 1, 185-224

DOI: https://doi.org/10.4213/im233

Использование Общероссийского математического портала Math-Net.Ru подразумевает, что вы прочитали и согласны с пользовательским соглашением http://www . mathnet.ru/rus/agreement

Параметры загрузки:

IP: 3.93 .64 .190

26 апреля 2023 г., 02:48:13 
УДК 512.6

\author{
С. Г. Танкеев
}

\title{
О весах $l$-адического представления и арифметике собственных чисел Фробениуса
}

Пусть $J$ - абсолютно простое абелево многообразие над числовым полем $k$, $[k: \mathbb{Q}]<\infty$. Предположим, что $\operatorname{Cent}(\operatorname{End}(J \otimes \bar{k}))=\mathbb{Z}$. Если $\mathbb{Q}$-алгебра с делением $\operatorname{End}^{0}(J \otimes \bar{k})$ расщепляется в простой точке $l$, то $l$-адическое представление определено микровесами простых классических алгебр Ли типов $A_{m}, B_{m}, C_{m}$ или $D_{m}$.

Если $S$ - поверхность типа К3 над достаточно большим числовым полем $k \subset \mathbb{C}$ и группа Ходжа $\operatorname{Hg}\left(S \otimes_{k} \mathbb{C}\right)$ полупроста, то $S$ имеет обыкновенную редукцию в каждой неархимедовой точке $k$ из некоторого множества с плотностью Дирихле 1.

Если $J$ - абсолютно простое трехмерное абелево многообразие типа IV по классификации Альберта над достаточно большим числовым полем, то $J$ имеет обыкновенную редукцию в каждой точке из некоторого множества с плотностью Дирихле 1.

Библиография: 35 наименований.

\section{Введение}

0.1. Пусть $J$ - абелево многообразие над числовым полем $k \subset \mathbb{C},[k: \mathbb{Q}]<\infty$. Для данного простого числа $l$ рассмотрим естественное $l$-адическое представление

$$
\rho_{l}: \operatorname{Gal}(\bar{k} / k) \rightarrow \operatorname{GL}\left(H_{\mathrm{et}}^{1}\left(J \otimes \bar{k}, \mathbb{Q}_{l}\right)\right)
$$

Мы обозначаем через

$$
\rho_{l}^{\wedge 2}: \operatorname{Gal}(\bar{k} / k) \rightarrow \operatorname{GL}\left(H_{\mathrm{et}}^{2}\left(J \otimes \bar{k}, \mathbb{Q}_{l}\right)\right)=\mathrm{GL}\left(\wedge^{2} H_{\mathrm{et}}^{1}\left(J \otimes \bar{k}, \mathbb{Q}_{l}\right)\right)
$$

естественное $l$-адическое представление во вторых когомологиях $J \otimes \bar{k}$.

Напомним некоторые гипотезы и результаты, касающиеся $\rho_{l}$.

Работа написана при финансовой поддержке Российского фонда фундаментальных исследований (грант 97-01-00647) и Центра грантов Новосибирского государственного университета. Исследование в MSRI выполнено при частичной поддержке Национального научного фонда (грант DMS-9022140). Статья подготовлена к печати во время стажировки автора в Институте высших научных исследований (Бюр-сюр-Иветт) и в Институте математических исследований MSRI (Беркли) весной 1997 г. Автор хотел бы поблагодарить сотрудников институтов за их теплое гостеприимство и превосходные условия для работы.

$$
\text { (C) С.Г. ТАнкеEв, } 1999
$$


0.2. ГипотезА (Серра-Мамфорда-Тейта [11], [19]). Существует канонический изоморфизм алгебр Ли

$$
\operatorname{Lie} \operatorname{Im}\left(\rho_{l}\right) \simeq \operatorname{Lie}\left[\operatorname{MT}\left(J \otimes_{k} \mathbb{C}\right)\left(\mathbb{Q}_{l}\right)\right]
$$

где $\operatorname{MT}\left(J \otimes_{k} \mathbb{C}\right)$ - группа Мамфорда-Тәйта, ассоциированная с канонической $\mathbb{Q}$-структурой Ходжа на $H^{1}\left(J \otimes_{k} \mathbb{C}, \mathbb{Q}\right)$.

0.3. Теорема (Делиня [35, теорема $0.5 .1 \mathrm{~b}])$. Любой простой фактор g редуктивной алгебры Ли Lie $\mathrm{MT}\left(J \otimes_{k} \mathbb{C}\right) \otimes \mathbb{C}$ является классической алгеброй Ли типа $A_{m}, B_{m}, C_{m}$ или $D_{m}$, и стариий вес любого неприводимого g-подмодуля $E \subset H^{1}\left(J \otimes_{k} \mathbb{C}, \mathbb{Q}\right) \otimes_{\mathbb{Q}} \mathbb{C}$ является микровесом.

Напомним список микровесов:

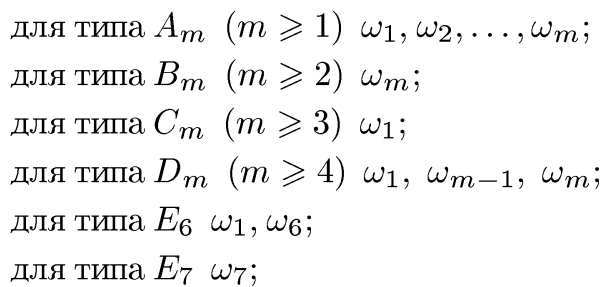

здесь $\omega_{1}, \omega_{2}, \ldots, \omega_{m}$ - фундаментальные веса [4, гл. $8, \S 7$, п. 3$]$.

В силу теоремы Делиня следующую гипотезу можно рассматривать как слабую форму гипотезы Серра-Мамфорда-Тэйта.

0.4. ГИпотеЗА (о микровесах $[32$, гипотеза 0.4]). Пусть $J$ - абелево многообразие над иисловым полем $k \subset \mathbb{C}$. Тогда любой простой фактор g редуктивной алгебры Ли $\operatorname{Lie} \operatorname{Im}\left(\rho_{l}\right) \otimes \overline{\mathbb{Q}}_{l}$ является классической алгеброй Ли типа $A_{m}, B_{m}, C_{m}$ или $D_{m}$, и старший вес любого неприводимого $g$-подмодуля $E \subset H_{\mathrm{et}}^{1}\left(J \otimes \bar{k}, \mathbb{Q}_{l}\right) \otimes \overline{\mathbb{Q}}_{l}$ является микровесом.

0.5. Если $\operatorname{End}(J \otimes \bar{k})=\mathbb{Z}$ и $\operatorname{dim}_{k} J$ - нечетное число, то в силу хорошо известной теоремы Серра $[7$, п. 6.1] сушествует канонический изоморфизм $l$-адических алгебр Ли $\operatorname{Lie} \operatorname{Im}\left(\rho_{l}\right) \simeq \operatorname{Lie}\left[\operatorname{MT}\left(J \otimes_{k} \mathbb{C}\right)\left(\mathbb{Q}_{l}\right)\right] . \quad$ В частности, гипотеза Серра-Мамфорда-Тэйта и гипотеза о микровесах верны для $J$. Аналогичные результаты получены для некоторых простых абелевых многообразий четных размерностей [26]-[28].

В этой статье мы доказываем следуюшие теоремы.

0.6. Теорема. Пусть $J$ - абсолютно простое абелево многообразие над числовым полем $k,[k: \mathbb{Q}]<\infty$. Предположим, ито $\operatorname{Cent}(\operatorname{End}(J \otimes \bar{k}))=\mathbb{Z}$. Если $\mathbb{Q}$-алгебра с делением $\operatorname{End}^{0}(J \otimes \bar{k})$ расщепляется в простой точке $l$, то любой простой фактор g редуктивной алгебры Ли $\operatorname{Lie} \operatorname{Im}\left(\rho_{l}\right) \otimes \overline{\mathbb{Q}}_{l}$ является простой алгеброй Ли типа $A_{m}, B_{m}, C_{m}$ или $D_{m}$, и старший вес любого неприводимого g-подмодуля $E \subset H_{\mathrm{et}}^{1}\left(J \otimes \bar{k}, \mathbb{Q}_{l}\right) \otimes \overline{\mathbb{Q}}_{l}$ является микровесом. 
0.7. ЗАмЕчАнИЕ. Р.Пинк независимо от автора доказал гипотезу о микровесах для всех абелевых многообразий над числовыми полями [16, теорема (5.10), следствие (5.11)]. Его значительно более сложное доказательство основано на технике кристальных когомологий.

0.8. Доказательство теоремы 0.6 основано на классических результатах Ж.-П.Серра $[18, \S 3]$. Мы получаем из этих результатов и условий теоремы 0.6, что конфигурация множества $\Delta$ всех собственных чисел $\Phi$ робениуса, отвечающих достаточно общей неархимедовой точке $v$ поля $k$ в пространстве $\Gamma_{v} \otimes \mathbb{Q}$ (где $\Gamma_{v}$ - мультипликативная подгруппа $\overline{\mathbb{Q}}^{\times}$, порожденная $\Delta$ ), идентична конфигурации некоторого подмножества вершин куба в $\Gamma_{v} \otimes \mathbb{Q}$. Поскольку доказательство элементарно и содержит красивые геометрические конструкции, некоторые из этих конструкций, вероятно, могут быть полезными при решении других диофантовых задач.

0.9. Например, пусть $S$ - гладкая проективная поверхность типа К3 над достаточно большим числовым полем $k \subset \mathbb{C},[k: \mathbb{Q}]<\infty$. Для любого простого числа $l$ рассмотрим естественное $l$-адическое представление $\rho_{l}^{S}: \operatorname{Gal}(\bar{k} / k) \rightarrow$ $\mathrm{GL}\left(H_{\mathrm{et}}^{2}\left(S \otimes_{k} \bar{k}, \mathbb{Q}_{l}\right)\right.$. Известно, что существует канонический изоморфизм алгебр Ли $[25]$

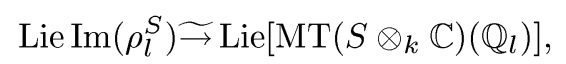

где $\operatorname{MT}\left(S \otimes_{k} \mathbb{C}\right)$ - группа Мамфорда-Тэйта, ассоциированная с естественной $\mathbb{Q}$-структурой Ходжа на $H^{2}\left(S \otimes_{k} \mathbb{C}, \mathbb{Q}\right)$. Этот результат существенно обобщен в [1].

Пусть $V_{\mathbb{Q}}(1)$ - ортогональное дополнение $\operatorname{NS}\left(S \otimes_{k} \mathbb{C}\right) \subset H^{2}\left(S \otimes_{k} \mathbb{C}, \mathbb{Q}(1)\right)$ относительно индекса пересечения. Группа Ходжа $\operatorname{Hg}\left(S \otimes_{k} \mathbb{C}\right)$ естественным образом действует на $V_{\mathbb{Q}}$. В силу хорошо известной теоремы Ю. Г. Зархина $K=$ $\operatorname{End}_{\mathrm{Hg}\left(S \otimes_{k} \mathbb{C}\right)} V_{\mathbb{Q}}$ является вполне вешественным числовым полем или чисто мнимым квадратичным расширением вполне вешественного поля $K_{0}$. Более того, $K$ является вполне вешественным полем тогда и только тогда, когда $\operatorname{Hg}\left(S \otimes_{k} \mathbb{C}\right)$ полупростая алгебраическая $\mathbb{Q}$-группа [34].

Предположим, что $S$ имеет хорошую редукцию в неархимедовой точке $v$ поля $k$. Пусть $F_{\bar{v}} \in \operatorname{Gal}(\bar{k} / k)$ - элемент Фробениуса, ассоциированный с точкой $\bar{v}$ поля $\bar{k}$, лежащей над точкой $v$. Мы говорим, что $S$ имеет обыкновенную редукиию в неархимедовой точке $v$ поля $k$ с полем вычетов $k(v)=\mathbb{F}_{q_{v}}$ характеристики $p_{v} \neq l$, если и только если специальный слой $S_{v}$ является гладкой проективной поверхностью типа К 3 , и для любого собственного числа $\delta$ линейного оператора $\rho_{l}^{S}\left(F_{\bar{v}}^{-1}\right)$ и для любой точки $w$ поля $\overline{\mathbb{Q}}$ над $p_{v}$ выполняется соотношение

$$
w(\delta) / w\left(q_{v}\right) \in\{0,1,2\} .
$$

Пусть $T$ - множество неархимедовых точек поля $k$. Напомним, что плотность Дирихле $T$ во множестве всех неархимедовых точек $k$ определяется как

$$
\lim _{x \rightarrow \infty} \frac{\log x}{x} \operatorname{Card}\left(\left\{v \in T \mid \operatorname{Norm}_{k / \mathbb{Q}}(v) \leqslant x\right\}\right)
$$

(если такой предел сушествует). 
Если $J$ - абелево многообразие размерности $\leqslant 2$ над достаточно большим числовым полем $k$, то плотность Дирихле множества $\{v \mid v$-неархимедова точка $k$, $J$ имеет обыкновенную редукцию в $v\}$ равна 1 согласно результату А. Огаса [15]. Это же верно для абелева многообразия без комплексного умножения при условии, что $\operatorname{Lie} \operatorname{Hg}\left(J \otimes_{k} \mathbb{C}\right) \otimes \mathbb{C}$ имеет тип $A_{1} \times A_{1} \times \cdots \times A_{1}[14]$ или если $\operatorname{End}\left(J \otimes_{k} \mathbb{C}\right)=\mathbb{Z}$ и $\operatorname{Lie} \mathrm{Hg}\left(J \otimes_{k} \mathbb{C}\right) \otimes \mathbb{C}$ не имеет простых факторов типа $C_{r},(r \geqslant 3)$ [16, следствие (7.2)].

В этой статье мы выводим из (0.9.1) следуюший результат.

0.10. ТЕОРЕМА. Пусть $S$ - гладкая проективная поверхность типа К3 над достаточно большим числовым полем $k,[k: \mathbb{Q}]<\infty$. Если ранг группь Нерона-Севери $S \otimes_{k} \mathbb{C}$ является нечетнылм числом или если группа Ходжа $\operatorname{Hg}\left(S \otimes_{k} \mathbb{C}\right)$ - полупростая алгебраическая $\mathbb{Q}$-группа, то множество всех неархимедовых точек $k$, в которых $S$ имеет обыкновенную редукцию, является множеством плотности 1 по Дирихле.

При условиях теоремы 0.10 множество $\Delta$ собственных чисел $\Phi$ робениуса в первых когомологиях абелева многообразия Куги-Сатаке имеет конфигурацию подмножества множества всех вершин куба в $\Gamma_{v} \otimes \mathbb{Q}$ (для достаточно общей неархимедовой точки $v$ поля $k$ ). Поэтому можно применить технику Р. Нута [14] в этой новой ситуации.

0.11. Напомним, что абелево многообразие $J$ имеет обыкновенную редукцию в неархимедовой точке $v$ поля $k$ с полем вычетов $k(v)=\mathbb{F}_{q_{v}}$ характеристики $p_{v}$, если и только если специальный слой $J_{v}$ минимальной модели Нерона $J$ является абелевым многообразием и выполняются следующие эквивалентные условия:

1) $p_{v}$-ранг $J_{v}$ равен $\operatorname{dim}_{k(v)} J_{v}$

$2)$ для любого собственного числа $\delta$ оператора $\rho_{l}\left(F_{\bar{v}}^{-1}\right), l \neq p_{v}$, и для любой точки $w$ поля $\overline{\mathbb{Q}}$, лежашей над $p_{v}$, выполнено соотношение

$$
w(\delta) / w\left(q_{v}\right) \in\{0,1\}
$$

3) редукция по модулю $p_{v}$ характеристического полинома $\operatorname{Pc}_{J}\left(\rho_{l}\left(F_{\bar{v}}^{-1}\right), t\right)$ не делится на $t^{d+1}$, где $d=\operatorname{dim}_{k} J[8$, п. 2].

Напомним, что $J \otimes \bar{k}$ - абелево многообразие типа IV по классификации Альберта, если и только если $K=\operatorname{Cent}\left(\operatorname{End}^{0}(J \otimes \bar{k})\right)$ является мнимым квадратичным расширением вполне вешественного поля. Более того, если $[K: \mathbb{Q}]=2 \cdot \operatorname{dim}_{k} J$, то $J$ - абелево многообразие СМ-типа [8, $\S 22]$.

Доказательство следуюшей теоремы основано на анализе разложений простых чисел в расширениях степени 3 или 6 поля $\mathbb{Q}$ (которые не являются, вообще говоря, нормальными, и поэтому в данной ситуации нет хороших симметрий) и на следующем замечательном диофантовом свойстве следа Фробениуса: если $J$ - абелево многообразие размерности $\geqslant 2$ над достаточно большим числовым полем $k$, то характеристика $p_{v}$ поля вычетов $k(v)$ не делит $\operatorname{Tr}\left(\rho_{l}^{\wedge}\left(F_{\bar{v}}^{-1}\right)\right)$ для всех неархимедовых точек $k$, лежаших в некотором подмножестве точек с плотностью Дирихле 1 [26, теорема 0.4].

0.12. Теорема. Пусть $J$ - абсолютно простое трехмерное абелево многообразие типа IV над достаточно больиим числовым полем $k,[k: \mathbb{Q}]<\infty$. 
Тогда специальный слой $J_{v}$ минимальной модели Нерона $J$ является обыкновенным абелевым многообразием для любой неархимедовой точки $v$ поля $k$ из некоторого множества с плотностью Дирихле 1.

Доказательства результатов этой статьи содержатся в препринтах [29]-[31].

Автор благодарен Ж.-П. Серру, Д. Бертрану и И. Андре за стимулирующее обсуждение этой темы.

\section{$\S 1$. Некоторые замечания об $l$-адическом представлении}

1.1. Всюду в дальнейшем $J$ - абелево многообразие над числовым полем $k$, $[k: \mathbb{Q}]<\infty, V_{l}=H_{\mathrm{et}}^{1}\left(J \otimes \bar{k}, \mathbb{Q}_{l}\right)$. Пусть $G_{V_{l}}$ - алгебраическая оболочка компактной $l$-адической группы Ли $\operatorname{Im}\left(\rho_{l}\right)$. Известно, что $G_{V_{l}}$ совпадает с замыканием $\operatorname{Im}\left(\rho_{l}\right)$ в топологии Зариского групшы $\mathrm{GL}\left(V_{l}\right)$.

Фиксируем неархимедову точку $v$ поля $k$. Пусть $k(v)$ - ее поле вычетов характеристики $p_{v}, k_{v}$ - пополнение поля $k$ относительно топологии, индуцированной точкой $v, \bar{v}$-продолжение $v$ на поле $\bar{k}, D(\bar{v})$ - группа разложения, $I(\bar{v}) \subset \operatorname{Gal}(\bar{k} / k)$ - группа инерции.

1.2. Предположим, что $v$ - неархимедова точка поля $k$, в которой $J$ имеет хорошую редукцию. Пусть $F_{\bar{v}} \in \mathrm{Gal}(\bar{k} / k)$ - элемент Фробениуса. Известно, что характеристический полином $\rho_{l}\left(F_{\bar{v}}^{-1}\right)$ совпадает с характеристическим полиномом эндоморфизма Фробениуса $\pi_{v}$ редукции $J_{v}$ многообразия $J$ в точке $v$. Обозначим через $\Delta$ множество всех собственных чисел $\rho_{l}\left(F_{\bar{v}}^{-1}\right)$ (с учетом кратностей). Пусть $\Gamma_{v}$ - мультипликативная подгруппа $\overline{\mathbb{Q}}^{\times}$, порожденная $\Delta$.

По теории Хонды-Тэйта [32] $\mathbb{Q}\left[\pi_{v}\right]=\prod K_{i}, K_{i}$ - числовые поля. Мультипликативная группа $\mathbb{Q}\left[\pi_{v}\right]^{\times}$определяет $\mathbb{Q}$-тор $T_{\pi_{v}}=\prod R_{K_{i} / \mathbb{Q}}\left(\mathrm{G}_{m K_{i}}\right)$, где $R_{K_{i} / \mathbb{Q}}$ - ограничения скаляров по Вейлю. Пусть $H_{v}$ - наименьшая алгебраическая подгруппа $T_{\pi_{v}}$, определенная над $\mathbb{Q}$, для которой $\pi_{v} \in H_{v}(\mathbb{Q})$. Как хорошо известно, $H_{v}$ - группа мультипликативного типа. Связная компонента единицы в $H_{v}$ называется тором фробениуса $T_{v}$. Кроме того, $T_{v}$ является $\mathbb{Q}$-тором в $G_{V_{l}}[7$, п. $3 \mathrm{~b}]$.

1.3. Обозначим через $\mathbb{C}_{p_{v}}=\left(\overline{\mathbb{Q}}_{p_{v}}\right)^{\wedge}$ пополнение алгебраического замыкания поля $\mathbb{Q}_{p_{v}}$. Согласно Тэйту и Рейно сушествует каноническое разложение Ходжа-Тэйта

$$
V_{\mathbb{C}_{p_{v}}}=V_{p_{v}} \otimes_{\mathbb{Q}_{p_{v}}} \mathbb{C}_{p_{v}}=V_{\mathbb{C}_{p_{v}}}(0) \oplus V_{\mathbb{C}_{p_{v}}}(-1)
$$

и $\operatorname{dim} V_{\mathbb{C}_{p_{v}}}(0)=\operatorname{dim} V_{\mathbb{C}_{p_{v}}}(-1)[7$, п. 4.3].

Определим морфизм алгебраических $\mathbb{C}_{p_{v}}$-групп

$$
h_{V_{p_{v}}}: \mathrm{G}_{m \mathbb{C}_{p_{v}}} \rightarrow \mathrm{GL}\left(V_{\mathbb{C}_{p_{v}}}\right)
$$

формулой

$$
h_{V_{p_{v}}}(c)(x)=\left\{\begin{array}{lll}
x, & \text { если } & x \in V_{\mathbb{C}_{p_{v}}}(0), \\
c^{-1} x, & \text { если } x \in V_{\mathbb{C}_{p_{v}}}(-1) .
\end{array}\right.
$$

Алгебраическая оболочка $\rho_{p_{v}}(I(\bar{v}))$ является алгебраической подгруппой $G_{V_{p_{v}}}$. По теореме Сена [17] 1-параметрическая подгруппа $h_{V_{p_{v}}}$ содержится в алгебраической оболочке $\rho_{p_{v}}(I(\bar{v}))$. В частности, $\operatorname{Im}\left(h_{V_{p_{v}}}\right) \subset G_{V_{p_{v}}}\left(\mathbb{C}_{p_{v}}\right)$. 
1.4. Лемма. Предположим, ито $\operatorname{Cent}_{\operatorname{End}^{0}}(J \otimes \bar{k})$ - вполне вещественное поле и $J(k)$ содержит все точки порядка $p_{v}^{2}$ в $J(\bar{k})$. Пусть $M \subset G_{V_{p_{v}}}$ наименьшая нормальная алгебраическая подгруппа, для которой $\operatorname{Im}\left(h_{V_{p_{v}}}\right) \subset$ $M\left(\mathbb{C}_{p_{v}}\right)$. Тогда каноническое представление полупростой алгебры Ли $[\operatorname{Lie}(M)]^{s s} \otimes \overline{\mathbb{Q}}_{p_{v}}$ в $V_{p_{v}} \otimes \overline{\mathbb{Q}}_{p_{v}}$ определено микровесами классических алгебр Ли (типов $\left.A_{m}, B_{m}, C_{m}, D_{m}\right)$.

ДоказАтельство. По теореме $\Phi$.А. Богомолова [2] $\operatorname{Lie} \operatorname{Im}\left(\rho_{p_{v}}\right)=\operatorname{Lie}\left(G_{V_{p_{v}}}\right)$, и $G_{V_{p_{v}}}$ содержит группу $\mathrm{G}_{m}$ гомотетий. В силу результатов $\Gamma$. Фалтингса [10] группа $G_{V_{p_{v}}}$ редуктивна. В рассматриваемом случае $G_{V_{p_{v}}}$ связна $[7$, предложение 3.6] и $G_{V_{p_{v}}}=\mathrm{G}_{m} \cdot S_{V_{p_{v}}}$, где $S_{V_{p_{v}}}=\left[G_{V_{p_{v}}}, G_{V_{p_{v}}}\right]$ - коммутант $G_{V_{p_{v}}}[7$, п. $1.2 .2 \mathrm{~b}]$. Ясно, что $M$ - связная редуктивная алгебраическая группа над $\mathbb{Q}_{p_{v}}$ с нетривиальной полупростой частью $M^{s s}=[M, M]$ (потому что $\operatorname{Im}\left(h_{V_{p_{v}}}\right)$ не содержится в $\left.\mathrm{G}_{m}\left(\mathbb{C}_{p_{v}}\right)\right)$; каноническое представление $V_{p_{v}}$ группы $M$ является точным. Если $N$ - нормальная связная алгебраическая подгруппа $M$, определенная над $\mathbb{Q}_{p_{v}}$, для которой $\operatorname{Im}\left(h_{V_{p_{v}}}\right) \subset N\left(\mathbb{C}_{p_{v}}\right)$, то $N$ - нормальная алгебраическая подгруппа $G_{V_{p_{v}}}$. Действительно, пусть $f: S_{V_{p_{v}}}^{s c} \rightarrow S_{V_{p_{v}}}-$ универсальное накрытие; поскольку $M^{s s}$ - нормальная алгебраическая подгруппа $S_{V_{p_{v}}}$, группа $\left(f^{-1}\left(M^{s s}\right)\right)^{0}$ является нормальной алгебраической подгруппой $S_{V_{p_{v}}}^{s c}$, и поэтому мы получаем разложение $S_{V_{p_{v}}}^{s c}=\left(f^{-1}\left(M^{s s}\right)\right)^{0} \times H^{\prime \prime}$, где $\left(f^{-1}\left(M^{s s}\right)\right)^{0}$ и $H^{\prime \prime}$ - односвязные полупростые $\mathbb{Q}_{p_{v}}$-группы; с другой стороны, $\left(f^{-1}\left(N^{s s}\right)\right)^{0}$ - нормальная связная подгруппа $\left(f^{-1}\left(M^{s s}\right)\right)^{0}$, поэтому мы получаем разложения $\left(f^{-1}\left(M^{s s}\right)\right)^{0}=\left(f^{-1}\left(N^{s s}\right)\right)^{0} \times H^{\prime}$ и $S_{V_{p_{v}}}^{s c}=\left(f^{-1}\left(N^{s s}\right)\right)^{0} \times H^{\prime} \times H^{\prime \prime} ;$ следовательно, $N^{s s}$ - нормальная алгебраическая подгруппа $S_{V_{p_{v}}}$, и $N$ - нормальная алгебраическая подгруппа $G_{V p_{v}}$. Поэтому $N=M$. Действие $\mathrm{G}_{m \mathbb{C}_{p_{v}}}$ на $V_{\mathbb{C}_{p_{v}}}=V_{p_{v}} \otimes_{\mathbb{Q}_{p_{v}}} \mathbb{C}_{p_{v}}$, определенное $h_{V_{p_{v}}}$, имеет веса 0 и -1 . В силу известных результатов Ж.-П. Серра $[18, \S 3]$ представление $V_{p_{v}}$ группы $M$ определено микровесами классических алгебр Ли. Лемма доказана.

1.5. СледСтвиЕ. Предположим, ито $\operatorname{Cent}_{\operatorname{End}^{0}}(J \otimes \bar{k})$ - вполне вещественное поле. Тогда для любого простого числа l редуктивная алгебра Ли $\operatorname{Lie} \operatorname{Im}\left(\rho_{l}\right) \otimes \overline{\mathbb{Q}}_{l}$ имеет такой простой фактор g, ито каноническое представление $g$ в $V_{l} \otimes \overline{\mathbb{Q}}_{l}$ определено микровесами классических алгебр Ли.

Действительно, мы можем выбрать такую неархимедову точку $v$, что $p_{v}=l$. Заменив $k$ некоторым конечным алгебраическим расширением, мы можем считать, что $J(k)$ содержит все точки порядка $p_{v}^{2}$ в $J(\bar{k})$.

\section{§ 2. $\mathrm{O} \mathrm{Gal}(\overline{\mathbb{Q}} / \mathbb{Q})$-инвариантных подмножествах $\Delta \cdot \Delta$}

2.1. ПРЕДЛОЖЕНИЕ. Пусть $J$ - абелево многообразие размерности $\geqslant 2$ над числовым полем $k$. После замены $k$ некоторым конечным расширением можно считать, что для абелева многообразия $J$ и для любой неархимедовой точки $v$ поля $k$ из некоторого множества $S_{\text {non-Arch }}^{\text {generic }}(J, k, l)$ точек с плотностью Дирихле 1 выполняются следующие условия:

1) для фиксированного иелого числа $n \geqslant 2$ с условием $l^{n}>\left(2 \cdot \operatorname{dim}_{k} J\right)^{2}$ все точки $l^{n}$-кручения в $J(\bar{k})$ рачиональны над $k, u k$ содержит все корни степени $l^{n}$ из единицы; 
2) $p_{v}=\operatorname{char}(k(v))>\left(2 \cdot \operatorname{dim}_{k} J\right)^{2}$;

3) $\operatorname{Norm}_{k / \mathbb{Q}}(v)=p_{v} ; p_{v}$ не разветвлено в $k$;

4) $J$ имеет хорошую редукцию в $v ;$ следы Фробениуса $\operatorname{Tr}\left(\rho_{l}\left(F_{\bar{v}}^{-1}\right)\right) \quad u$ $\operatorname{Tr}\left(\rho_{l}^{\wedge}\left(F_{\bar{v}}^{-1}\right)\right)$ не делятся на $p_{v} ;$

5) $\Gamma_{v}$ не имеет кручения, $G_{V_{l}}$ связна и $\rho_{l}\left(F_{\bar{v}}^{-1}\right) \in T_{v}(\mathbb{Q})$;

6) тор Фробениуса $T_{v}$ является максимальным тором в $G_{V_{l}}$ u

$$
\operatorname{rank}\left(\Gamma_{v}\right)=\operatorname{dim}\left(T_{v}\right)=\operatorname{rank}\left(G_{V_{l}}\right)
$$

ДокАЗАТЕЛЬСТВО. После замены $k$ некоторым конечным расширением можно считать, что для абелева многообразия $J$ и для $k$ выполняется условие 1$)$. Тогда плотность Дирихле множества $S_{\text {non-Arch }}^{\text {generic }}(J, k, l)$ всех неархимедовых точек $k$, удовлетворяющих условиям 2)-6), равна 1 (см. [7, лемма 2.1 , предложения $3.6,(5.2 .1)$, следствие 3.8$]$; [26, теорема 0.4]). Предложение доказано.

В дальнейшем мы считаем, что $v \in S_{\text {non-Arch }}^{\text {generic }}(J, k, l), \operatorname{Cent} \operatorname{End}(J \otimes \bar{k})=\mathbb{Z}$ и $\mathbb{Q}$-алгебра с делением $\operatorname{End}^{0}(J \otimes \bar{k})$ расщепляется в простой точке $l$. В этом случае $G_{V_{l}}=\mathrm{G}_{m} \cdot S_{V_{l}}$, где $S_{V_{l}}=\left[G_{V_{l}}, G_{V_{l}}\right]$ - коммутант $G_{V_{l}}[7$, п. $1.2 .2 \mathrm{~b}]$. Более того, по классификации Альберта $[13, \S 21] V_{l}$ является прямой суммой 1 или 2 копий абсолютно неприводимого симплектического или ортогонального $S_{V_{l}}$-модуля $U_{l}$.

2.2. Пусть $f: S_{V_{l}}^{s c} \rightarrow S_{V_{l}}$ - универсальное накрытие. Изогения $f$ продолжается до изогении $f_{\mathrm{G}_{m}}: \mathrm{G}_{m} \times S_{V_{l}}^{s c} \rightarrow \mathrm{G}_{m} \cdot S_{V_{l}}=G_{V_{l}}$, определенной формулой $f_{\mathrm{G}_{m}}(a \times$ $s)=a \cdot f(s)$ при $a \in \mathrm{G}_{m}, s \in S_{V_{l}}^{s c}$.

Рассмотрим точную последовательность

$$
1 \rightarrow \operatorname{Ker}\left(f_{\mathrm{G}_{m}}\right) \rightarrow \mathrm{G}_{m} \times S_{V_{l}}^{s c} \rightarrow \mathrm{G}_{m} \cdot S_{V_{l}}=G_{V_{l}} \rightarrow 1
$$

Она определяет точную последовательность коммутативных алгебраических $\mathbb{Q}_{l}$-групп

$$
1 \rightarrow \operatorname{Ker}\left(f_{\mathrm{G}_{m}}\right) \cap\left[f_{\mathrm{G}_{m}}^{-1}\left(T_{v}\right)\right]^{0} \rightarrow\left[f_{\mathrm{G}_{m}}^{-1}\left(T_{v}\right)\right]^{0} \stackrel{\varphi}{\longrightarrow} T_{v} \rightarrow 1,
$$

где $\left[f_{\mathrm{G}_{m}}^{-1}\left(T_{v}\right)\right]^{0}$ - максимальный $\mathbb{Q}_{l}$-подтор $\mathrm{G}_{m} \times S_{V_{l}}^{s c}$, совпадаюший со связной компонентой единицы $f_{\mathrm{G}_{m}}^{-1}\left(T_{v}\right), \varphi$ является изогенией $\mathbb{Q}_{l}$-торов, $\operatorname{Ker}\left(f_{\mathrm{G}_{m}}\right) \cap$ $\left[f_{\mathrm{G}_{m}}^{-1}\left(T_{v}\right)\right]^{0}=\operatorname{Ker}(\varphi)-$ конечная группа, изоморфная некоторой подгруппе $\operatorname{Cent}\left(S_{V_{l}}^{s c}\right)$. Мы перепишем последовательность (2.2.1) как

$$
1 \rightarrow \operatorname{Ker}(\varphi) \rightarrow\left[f_{\mathrm{G}_{m}}^{-1}\left(T_{v}\right)\right]^{0} \stackrel{\varphi}{\longrightarrow} T_{v} \rightarrow 1
$$

2.3. Напомним, что $S_{V_{l}} \otimes \overline{\mathbb{Q}}_{l}$ является $\overline{\mathbb{Q}}_{l}$-почти простой алгебраической группой тогда и только тогда, когда $S_{V_{l}} \otimes \overline{\mathbb{Q}}_{l}$ полупроста и не имеет бесконечных нормальных алгебраических $\overline{\mathbb{Q}}_{l}$-подгрупп, отличных от $S_{V_{l}} \otimes \overline{\mathbb{Q}}_{l}$.

Если $S_{V_{l}} \otimes \overline{\mathbb{Q}}_{l}$ является $\overline{\mathbb{Q}}_{l}$-почти простой алгебраической группой, то в силу следствия $1.5 l$-адическое представление $\rho_{l}$ определено микровесами классических алгебр Ли. 
2.4. Предположим, что $S_{V_{l}} \otimes \overline{\mathbb{Q}}_{l}$ не является $\overline{\mathbb{Q}}_{l}$-почти простой алгебраической группой. Поскольку $S_{V_{l}}^{s c} \otimes \overline{\mathbb{Q}}_{l}-$ односвязная алгебраическая $\overline{\mathbb{Q}}_{l}$-группа, она является произведением двух собственных полупростых $\overline{\mathbb{Q}}_{l}$-подгрупп: $S_{V_{l}}^{s c} \otimes \overline{\mathbb{Q}}_{l}=S_{1} \times S_{2}$. Рассмотрим канонические проекции $\mathrm{pr}_{i}: \mathrm{G}_{m} \times S_{1} \times S_{2} \rightarrow S_{i}(i=1,2)$ и $\mathrm{pr}_{0}: \mathrm{G}_{m} \times S_{1} \times S_{2} \rightarrow \mathrm{G}_{m}$. Так как $\left[f_{\mathrm{G}_{m}}^{-1}\left(T_{v} \otimes \overline{\mathbb{Q}}_{l}\right)\right]^{0}$ - максимальный $\overline{\mathbb{Q}}_{l}$-подтор $\mathrm{G}_{m} \times S_{1} \times S_{2}$, мы имеем

$$
\begin{aligned}
& {\left[f_{\mathrm{G}_{m}}^{-1}\left(T_{v} \otimes \overline{\mathbb{Q}}_{l}\right)\right]^{0}} \\
& \quad=\operatorname{pr}_{0}\left(\left[f_{\mathrm{G}_{m}}^{-1}\left(T_{v} \otimes \overline{\mathbb{Q}}_{l}\right)\right]^{0}\right) \times \operatorname{pr}_{1}\left(\left[f_{\mathrm{G}_{m}}^{-1}\left(T_{v} \otimes \overline{\mathbb{Q}}_{l}\right)\right]^{0}\right) \times \operatorname{pr}_{2}\left(\left[f_{\mathrm{G}_{m}}^{-1}\left(T_{v} \otimes \overline{\mathbb{Q}}_{l}\right)\right]^{0}\right),
\end{aligned}
$$

и $\operatorname{pr}_{i}\left(\left[f_{\mathrm{G}_{m}}^{-1}\left(T_{v} \otimes \overline{\mathbb{Q}}_{l}\right)\right]^{0}\right)$ - максимальный $\overline{\mathbb{Q}}_{l}$-подтор $S_{i}, \quad(i=1,2), \operatorname{pr}_{0}\left(\left[f_{\mathrm{G}_{m}}^{-1}\left(T_{v} \otimes\right.\right.\right.$ $\left.\left.\left.\overline{\mathbb{Q}}_{l}\right)\right]^{0}\right)=\mathrm{G}_{m}$. Получаем разложение

$$
\left[f_{\mathrm{G}_{m}}^{-1}\left(T_{v} \otimes \overline{\mathbb{Q}}_{l}\right)\right]^{0}=\mathrm{G}_{m} \times \operatorname{pr}_{1}\left(\left[f_{\mathrm{G}_{m}}^{-1}\left(T_{v} \otimes \overline{\mathbb{Q}}_{l}\right)\right]^{0}\right) \times \operatorname{pr}_{2}\left(\left[f_{\mathrm{G}_{m}}^{-1}\left(T_{v} \otimes \overline{\mathbb{Q}}_{l}\right)\right]^{0}\right) .
$$

Можно найти такой элемент $y_{v} \in\left(\left[f_{\mathrm{G}_{m}}^{-1}\left(T_{v} \otimes \overline{\mathbb{Q}}_{l}\right)\right]^{0}\right)\left(\overline{\mathbb{Q}}_{l}\right)$, что $\varphi\left(y_{v}\right)=\rho_{l}\left(F_{\bar{v}}^{-1}\right)$ (напомним, что $\left.\rho_{l}\left(F_{\bar{v}}^{-1}\right) \in T_{v}(\mathbb{Q}) \subset T_{v}\left(\mathbb{Q}_{l}\right)\right)$. Поэтому разложение (2.4.1) дает разложение $y_{v}=\lambda \times y_{v 1} \times y_{v 2}$, где $\lambda \in \mathrm{G}_{m}\left(\overline{\mathbb{Q}}_{l}\right)=\overline{\mathbb{Q}}_{l} \times, y_{v i} \in \operatorname{pr}_{i}\left(\left[f_{\mathrm{G}_{m}}^{-1}\left(T_{v} \otimes\right.\right.\right.$ $\left.\left.\left.\overline{\mathbb{Q}}_{l}\right)\right]^{0}\right)\left(\overline{\mathbb{Q}}_{l}\right) \subset S_{i}\left(\overline{\mathbb{Q}}_{l}\right)$.

$\mathrm{C}$ другой стороны, $U_{l} \otimes \overline{\mathbb{Q}}_{l}=\overline{\mathbb{Q}}_{l} \otimes W_{1} \otimes W_{2}$, где $W_{1}$ - неприводимый $S_{1}$-модуль, $W_{2}$ - неприводимый $S_{2}$-модуль. Пусть $\rho_{0}: \mathrm{G}_{m} \otimes \overline{\mathbb{Q}}_{l} \rightarrow \mathrm{GL}\left(\overline{\mathbb{Q}}_{l}\right)=\overline{\mathbb{Q}}_{l}^{\times}, \rho_{i}: S_{i} \rightarrow$ $\mathrm{GL}\left(W_{i}\right) \quad(i=1,2)-$ соответствуюшие представления. Имеем коммутативную диаграмму

$$
\begin{array}{ccc}
\left(\mathrm{G}_{m} \otimes \overline{\mathbb{Q}}_{l}\right) \times S_{1} \times S_{2} \stackrel{\rho_{0} \otimes \rho_{1} \otimes \rho_{2}}{\longrightarrow} & \mathrm{GL}\left(\overline{\mathbb{Q}}_{l} \otimes W_{1} \otimes W_{2}\right) \\
& \downarrow f_{\mathrm{G}_{m}} & \| \\
G_{V_{l}} \otimes \overline{\mathbb{Q}}_{l} & \subset & \mathrm{GL}\left(\overline{\mathbb{Q}}_{l} \otimes W_{1} \otimes W_{2}\right) .
\end{array}
$$

Поскольку $f_{\mathrm{G}_{m}}\left(y_{v i}\right) \in T_{v}\left(\overline{\mathbb{Q}}_{l}\right) \subset \mathrm{GL}\left(U_{l}\right)\left(\overline{\mathbb{Q}}_{l}\right)$, то мы можем диагонализировать линейньй оператор $f_{\mathrm{G}_{m}}\left(y_{v i}\right)$ (над $\left.\overline{\mathbb{Q}}_{l}\right)$. Пусть $\Delta_{i}$ - множество его собственных чисел. Коммутативная диаграмма (2.4.2) показывает, что множество $\Delta$ всех собственных чисел линейного оператора $f_{\mathrm{G}_{m}}\left(y_{v}\right)=\varphi\left(y_{v}\right)=\rho_{l}\left(F_{\bar{v}}^{-1}\right)$ равно

$$
\Delta=\lambda \cdot \Delta_{1} \cdot \Delta_{2}
$$

с естественным действием $\mathrm{Gal}(\overline{\mathbb{Q}} / \mathbb{Q})$ на $\Delta$ (напомним, что характеристический полином $\rho_{l}\left(F_{\bar{v}}^{-1}\right)$ принадлежит $\left.\mathbb{Z}[t]\right)$. Пусть $\Gamma_{\Delta_{i}}-$ мультипликативная подгруппа $\overline{\mathbb{Q}}^{\times}$, порожденная $\Delta_{i}, \Gamma_{\{\lambda\}}=\left\{\lambda^{m} \mid m \in \mathbb{Z}\right\}$. Обозначим через $\mathrm{X}^{*}\left(\operatorname{pr}_{i}\left(\left[\varphi^{-1}\left(T_{v} \otimes\right.\right.\right.\right.$ $\left.\left.\left.\left.\overline{\mathbb{Q}}_{l}\right)\right]^{0}\right)\right)$ группу характеров $\overline{\mathbb{Q}}_{l}$-тора $\operatorname{pr}_{i}\left(\left[\varphi^{-1}\left(T_{v} \otimes \overline{\mathbb{Q}}_{l}\right)\right]^{0}\right)$. Очевидно, $\Gamma_{v} \subset \Gamma_{\{\lambda\}}$. $\Gamma_{\Delta_{1}} \cdot \Gamma_{\Delta_{2}}$, поэтому

$$
\begin{aligned}
\operatorname{rank} \Gamma_{v} & \leqslant \sum \operatorname{rankX}\left(\operatorname{pr}_{i}\left(\left[\varphi^{-1}\left(T_{v} \otimes \overline{\mathbb{Q}}_{l}\right)\right]^{0}\right)\right)\left(\operatorname{pr}_{i}\left(y_{v}\right)\right) \\
& \leqslant \sum \operatorname{rank} X^{*}\left(\operatorname{pr}_{i}\left(\left[\varphi^{-1}\left(T_{v} \otimes \overline{\mathbb{Q}}_{l}\right)\right]^{0}\right)\right. \\
& =\operatorname{rank}\left(\mathrm{G}_{m} \times S_{1} \times S_{2}\right)=\operatorname{rank}\left(G_{V_{l}}\right) .
\end{aligned}
$$


Условие 2.1,6) влечет соотношение

$$
\begin{aligned}
\operatorname{rank} X^{*} & \left(\operatorname{pr}_{i}\left(\left[\varphi^{-1}\left(T_{v} \otimes \overline{\mathbb{Q}}_{l}\right)\right]^{0}\right)\right)\left(\operatorname{pr}_{i}\left(y_{v}\right)\right) \\
= & \operatorname{rank} X^{*}\left(\operatorname{pr}_{i}\left(\left[\varphi^{-1}\left(T_{v} \otimes \overline{\mathbb{Q}}_{l}\right)\right]^{0}\right)\right)
\end{aligned}
$$

Поскольку существует каноническая точная последовательность

$$
\mathrm{X}^{*}\left(\operatorname{pr}_{i}\left(\left[\varphi^{-1}\left(T_{v} \otimes \overline{\mathbb{Q}}_{l}\right)\right]^{0}\right)\right) \rightarrow \mathrm{X}^{*}\left(\operatorname{pr}_{i}\left(\left[\varphi^{-1}\left(T_{v} \otimes \overline{\mathbb{Q}}_{L}\right)\right]^{0}\right)\right)\left(\operatorname{pr}_{i}\left(y_{v}\right)\right) \rightarrow 0,
$$

и $\mathrm{X}^{*}\left(\operatorname{pr}_{i}\left(\left[\varphi^{-1}\left(T_{v} \otimes \overline{\mathbb{Q}}_{l}\right)\right]^{0}\right)\right)$ не имеет кручения, мы получаем из (2.4.5), что

$$
\Gamma_{\Delta_{i}}=\mathrm{X}^{*}\left(\operatorname{pr}_{i}\left(\left[\varphi^{-1}\left(T_{v} \otimes \overline{\mathbb{Q}}_{l}\right)\right]^{0}\right)\right)\left(\operatorname{pr}_{i}\left(y_{v}\right)\right)
$$

не имеет кручения. Поэтому из (2.4.4) следует, что

$$
\Gamma_{\{\lambda\}} \cdot \Gamma_{\Delta_{1}} \cdot \Gamma_{\Delta_{2}}=\Gamma_{\{\lambda\}} \times \Gamma_{\Delta_{1}} \times \Gamma_{\Delta_{2}} .
$$

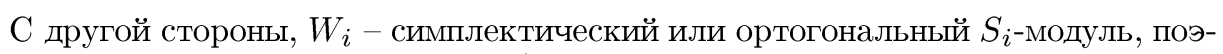
тому $W_{i}^{*}=W_{i}$. Следовательно, $\Delta_{i}^{-1}=\Delta_{i}$.

Группа Галуа $\operatorname{Gal}(\overline{\mathbb{Q}} / \mathbb{Q})$ действует естественным образом на $\Delta$ и на $\Delta \cdot \Delta$. Для любого элемента $\eta \in \Delta \cdot \Delta$ определим отображение $T_{\eta}: \Delta \rightarrow \overline{\mathbb{Q}}^{\times}$формулой $T_{\eta}(\delta)=$ $\eta \delta^{-1}$. Легко видеть, что для любого элемента $\sigma \in \mathrm{Gal}(\overline{\mathbb{Q}} / \mathbb{Q})$ мы имеем (с учетом кратностей $) \operatorname{Card}\left(T_{\eta}(\Delta) \cap \Delta\right)=\operatorname{Card}\left(T_{\sigma(\eta)}(\sigma(\Delta)) \cap \sigma(\Delta)\right)=\operatorname{Card}\left(T_{\sigma(\eta)}(\Delta) \cap \Delta\right)$. Поэтому для любой константы $c$ множество $\left\{\eta \in \Delta \cdot \Delta \mid \operatorname{Card}\left(T_{\eta}(\Delta) \cap \Delta\right)=c\right\}$ является $\operatorname{Gal}(\overline{\mathbb{Q}} / \mathbb{Q})$-инвариантным. Ясно, что

$$
\eta=\lambda^{2} \Leftrightarrow \operatorname{Card}\left(T_{\eta}(\Delta) \cap \Delta\right)=\operatorname{Card}(\Delta) .
$$

Следовательно, $\lambda^{2} \in \mathbb{Q}$ и $\lambda^{2}$ имеет абсолютную величину $p_{v}$ (по условию $2.1,3$ ) и в силу известной теоремы А. Вейля $[13, \S 21$, теорема 4$]$ ), поэтому мы получаем равенство $\lambda^{2}= \pm p_{v}$. Пусть $\rho$ - комплексное сопряжение на $\overline{\mathbb{Q}}$, индуцированное некоторым вложением $\overline{\mathbb{Q}} \rightarrow \mathbb{C}$. Согласно (2.4.6) любой элемент $\delta \in \Delta$ имеет единственное разложение $\delta=\lambda \cdot \delta_{1} \cdot \delta_{2}$, где $\delta_{i} \in \Delta_{i}$. Мы утверждаем, что

$$
\rho\left(\lambda \delta_{1} \delta_{2}\right)=\lambda \delta_{1}^{-1} \delta_{2}^{-1}
$$

Действительно, $\rho\left(\lambda \delta_{1} \delta_{2}\right)=\lambda \delta_{1}^{\prime} \delta_{2}^{\prime}$ для некоторого $\delta_{i}^{\prime} \in \Delta_{i}$. По теореме А. Вейля и в силу соотношения $2.1,3)$ имеем $\lambda \delta_{1} \delta_{2} \rho\left(\lambda \delta_{1} \delta_{2}\right)=p_{v}$; поэтому

$\lambda^{2} \delta_{1} \delta_{2} \delta_{1}^{\prime} \delta_{2}^{\prime}=p_{v}, \quad \lambda^{4}\left(\delta_{1} \delta_{1}^{\prime} \delta_{2} \delta_{2}^{\prime}\right)^{2}=p_{v}^{2}, \quad p_{v}^{2}\left(\delta_{1} \delta_{1}^{\prime} \delta_{2} \delta_{2}^{\prime}\right)^{2}=p_{v}^{2}, \quad\left(\delta_{1} \delta_{1}^{\prime} \delta_{2} \delta_{2}^{\prime}\right)^{2}=1$.

Заметим, что $\lambda^{2}=\lambda \delta_{1} \delta_{2} \cdot \lambda \delta_{1}^{-1} \delta_{2}^{-1} \in \Gamma_{v}$, поэтому $\delta_{1} \delta_{1}^{\prime} \delta_{2} \delta_{2}^{\prime}=\lambda \delta_{1} \delta_{2} \times$ $\lambda \delta_{1}^{\prime} \delta_{2}^{\prime} / \lambda^{2} \in \Gamma_{v}$. Поскольку $\Gamma_{v}$ не имеет кручения (по условию $\left.2.1,5\right)$ ), соотношение $\left(\delta_{1} \delta_{1}^{\prime} \delta_{2} \delta_{2}^{\prime}\right)^{2}=1$ влечет $\delta_{1}^{\prime} \delta_{2}^{\prime}=\left(\delta_{1} \delta_{2}\right)^{-1}$. Таким образом, утверждение $(2.4 .7)$ доказано.

Теорема А.Вейля и (2.4.7) показывают, что

$$
\lambda^{2}=\lambda \delta_{1} \delta_{2} \cdot \rho\left(\lambda \delta_{1} \delta_{2}\right)=p_{v}
$$

7

Серия математическая, №1 
2.5. Теорема. Предположим, что разложение $\Delta=\lambda \cdot \Delta_{1} \cdot \Delta_{2}$ соответствует разложсению $S_{V_{l}}^{s c} \otimes \overline{\mathbb{Q}}_{l}=S_{1} \times S_{2}$, әде $S_{1} u S_{2}$ - полупростые алгебраические $\overline{\mathbb{Q}}_{l}$-группь. Тогда для любого $i \Delta_{i} \cdot \Delta_{i}$ не содержит $\operatorname{Gal}(\overline{\mathbb{Q}} / \mathbb{Q})$-инвариантного подмноосества, не равного $\{1\}$ (без учета кратностей).

ДокАЗАТЕЛЬСТво. Предположим, что $\Delta_{1} \cdot \Delta_{1}$ содержит $\mathrm{Gal}(\overline{\mathbb{Q}} / \mathbb{Q})$-инвариантное подмножество $B \neq\{1\}$ (без учета кратностей). Очевидно,

$$
\operatorname{Card}(B)<\frac{1}{2} \cdot \operatorname{Card}(\Delta) \cdot \operatorname{Card}(\Delta) \leqslant \frac{1}{2} \cdot\left(2 \cdot \operatorname{dim}_{k} J\right)^{2}<\frac{1}{2} \cdot l^{n}
$$

Поскольку $\Delta_{2}^{-1}=\Delta_{2}$, то мы имеем $1 \in \Delta_{2} \cdot \Delta_{2}$, поэтому

$$
\lambda^{2} \cdot B \subset \lambda^{2} \cdot \Delta_{1} \cdot \Delta_{1} \subset \lambda^{2} \cdot \Delta_{1} \cdot \Delta_{1} \cdot \Delta_{2} \cdot \Delta_{2}=\Delta \cdot \Delta .
$$

Следовательно, $\sum_{z \in \lambda^{2} \cdot B} z \in \mathbb{Z}$.

Предположим, что $p_{v}$ не делит $\sum_{z \in \lambda^{2} \cdot B} z$. Тогда для любой точки $w$ поля $\overline{\mathbb{Q}}$, лежащей над $p_{v}$, имеем

$$
w\left(\sum_{z \in \lambda^{2} \cdot B} z\right)=0 .
$$

Следовательно, существует такой элемент $z_{w} \in \lambda^{2} \cdot B$, что $w\left(z_{w}\right)=0$. Поэтому для любого элемента $\delta_{2} \in \Delta_{2}$

$$
0=w\left(z_{w}\right)=\frac{1}{2}\left\{w\left(z_{w} \delta_{2}^{2}\right)+w\left(z_{w} \delta_{2}^{-2}\right)\right\}
$$

Поскольку оба слагаемые в последних скобках неотрицательные в силу соотношений $z_{w} \delta_{2}^{ \pm 2} \in \Delta \cdot \Delta$, мы имеем равенства $w\left(z_{w} \delta_{2}^{2}\right)=w\left(z_{w} \delta_{2}^{-2}\right)=0$. Поэтому $w\left(\delta_{2}\right)=0$ для всех $w \mid p_{v}$. Следовательно, $\delta_{2}-$ корень из 1 [35, подлемма 3.4.0]. Так как $\delta_{2} \in \Delta_{2}-$ произвольный элемент, мы заключаем, что мультипликативная группа $\Gamma_{\Delta_{2}}$, порожденная $\Delta_{2}$, является конечной подгруппой $\overline{\mathbb{Q}}^{\times}$. Мы знаем, что $\Gamma_{\Delta_{2}}$ не имеет кручения. Поэтому $\Gamma_{\Delta_{2}}=\{1\}$, и

$$
\begin{aligned}
\operatorname{rank} \Gamma_{v} \leqslant 1+\operatorname{rank} \Gamma_{\Delta_{1}} & =1+\operatorname{rankX}\left(\operatorname{pr}_{1}\left(\left[\varphi^{-1}\left(T_{v} \otimes \overline{\mathbb{Q}}_{l}\right)\right]^{0}\right)\right)\left(\operatorname{pr}_{1}\left(y_{v}\right)\right) \\
& \leqslant 1+\operatorname{rankX}\left(\operatorname{pr}_{1}\left(\left[\varphi^{-1}\left(T_{v} \otimes \overline{\mathbb{Q}}_{l}\right)\right]^{0}\right)\right) \\
& =1+\operatorname{rank}\left(S_{1}\right)<\operatorname{rank}\left(\mathrm{G}_{m} \times S_{1} \times S_{2}\right)=\operatorname{rank}\left(G_{V_{l}}\right)
\end{aligned}
$$

вопреки условию 2.1,6).

Следовательно, $p_{v}$ делит $\sum_{z \in \lambda^{2} \cdot B} z$. Так как $\lambda^{2}=p_{v}$ в силу (2.4.8) и каждый элемент $b \in B$ имеет абсолютную величину 1 в силу теоремы А.Вейля $[13, \S 21$, теорема 4], мы получаем соотношение

$$
\sum_{z \in \lambda^{2} \cdot B} z=p_{v} \cdot b_{v}
$$


где $b_{v} \in \mathbb{Z}$ имеет абсолютную величину $\leqslant \operatorname{Card}(B)<\frac{1}{2} \cdot l^{n}$ в силу (2.5.1).

По условию $2.1,3) p_{v}$ неразветвлено в $k$, и $\operatorname{Norm}_{k} / \mathbb{Q}(v)=p_{v}$. Следовательно, $p_{v}$ полностью распадается в $k$, и мы имеем

$$
k \otimes \mathbb{Q} \mathbb{Q}_{p_{v}} \simeq \mathbb{Q}_{p_{v}} \times \cdots \times \mathbb{Q}_{p_{v}} .
$$

По условию $2.1,1) k$ содержит все корни степени $l^{n}$ из единицы. С другой стороны, известно, что $\left(\mathbb{Q}_{p_{v}}^{\times}\right)_{\text {tors }} \simeq \mathbb{Z} /\left(p_{v}-1\right) \mathbb{Z}$. Из (2.5.2) следует, что $l^{n} \mid\left(p_{v}-1\right)$, и поэтому

$$
p_{v}=1 \quad\left(\bmod l^{n}\right) .
$$

По условию $2.1,1)$ все точки $l^{n}$-кручения в $J(\bar{k})$ рациональны над $k$. Следовательно, $\left.\rho_{l}\right|_{\mathrm{Gal}(\bar{k} / k)}$ тривиально $\bmod l^{n}$, и поэтому для всех $\delta \in \Delta$ мы имеем соотношение $\delta=1\left(\bmod l^{n}\right)$. Это же соотношение выполняется для любого элемента $\Delta \cdot \Delta$.

Известное соотношение $\lambda^{2} \cdot B \subset \Delta \cdot \Delta$ влечет

$$
\sum_{z \in \lambda^{2} \cdot B} z=\operatorname{Card}(B) \quad\left(\bmod l^{n}\right) .
$$

В силу (2.5.3) мы получаем из (2.5.4), что $b_{v}=\operatorname{Card}(B)\left(\bmod l^{n}\right)$. Ясно, что неравенства $\left|b_{v}\right| \leqslant \operatorname{Card}(B)<\frac{1}{2} \cdot l^{n}$ дают соотношение $b_{v}=\operatorname{Card}(B)$. Следовательно, все элементы множества $\lambda^{2} \cdot B$ равны $p_{v}=\lambda^{2}$ вопреки условию $B \neq\{1\}$. Теорема доказана.

2.6. В силу условия $2.1,5) \Gamma_{v}$ не имеет кручения. Поэтому мы можем рассматривать канонические вложения $\Delta \subset \Gamma_{v} \subset \Gamma_{v} \otimes \mathbb{Q}$. В силу следствия 1.5 сушествует такая простая односвязная алгебраическая $\overline{\mathbb{Q}}_{l}$-группа $S_{2}$ классического типа, что старший вес любого неприводимого $S_{2}$-подмодуля в $H_{\mathrm{et}}^{1}\left(J \otimes \bar{k}, \mathbb{Q}_{l}\right) \otimes \overline{\mathbb{Q}}_{l}$ является микровесом.

2.7. Лемма. Множество $\Delta_{2}$ не содержит трех различных точек, лежсащих на одной аффинной прямой пространства $\Gamma_{\Delta_{2}} \otimes \mathbb{Q}$.

ДокАЗАТЕЛЬСТво. Предположим, что $S_{2}$ - простая группа типа $A_{m}(m \geqslant 1)$. Тогда в обозначениях Н. Бурбаки

$$
\omega_{i}=\varepsilon_{1}+\cdots+\varepsilon_{i}-i \cdot\left(\varepsilon_{1}+\cdots+\varepsilon_{m+1}\right) /(m+1),
$$

группа Вейля $W(R)$ является группой всех перестановок $\left\{\varepsilon_{1}, \ldots, \varepsilon_{m+1}\right\}[4$, гл. 6 , п. 4.7]; $E\left(\omega_{r}\right)$ - симплектическое или ортогональное представление, если и только если $r=(m+1) / 2[4$, гл. 8 , таблица 1$]$, и в этом случае

$$
\begin{aligned}
& \omega_{r}=\left(\varepsilon_{1}+\cdots+\varepsilon_{(m+1) / 2}-\varepsilon_{(m+3) / 2}-\ldots-\varepsilon_{m+1}\right) / 2, \\
& \operatorname{ch} E\left(\omega_{r}\right)=\operatorname{ch} E\left(\omega_{(m+1) / 2}\right)=\sum_{\begin{array}{c}
a_{i} \in\{ \pm 1\} \\
a_{1}+\cdots+a_{m+1}=0
\end{array}} e^{a_{1} \varepsilon_{1} / 2+\cdots+a_{m+1} \varepsilon_{m+1} / 2} \\
& =\sum_{\substack{a_{i} \in\{ \pm 1\} \\
a_{1}+\cdots+a_{m} \in\{ \pm 1\}}} e^{a_{1}\left(\varepsilon_{1}-\varepsilon_{m+1}\right) / 2+\cdots+a_{m}\left(\varepsilon_{m}-\varepsilon_{m+1}\right) / 2} .
\end{aligned}
$$


Так как представление $S_{2}$ в $W_{2}$ является симплектическим или ортогональным, мы имеем следующее соотношение: $\Delta_{2}=\left\{\alpha_{1}^{a_{1}} \ldots \alpha_{m}^{a_{m}} \mid a_{j} \in\{ \pm 1\}, a_{1}+\cdots\right.$ $\left.\cdots+a_{m} \in\{ \pm 1\}\right\}$, где $\alpha_{1}, \alpha_{2}, \ldots, \alpha_{m}$ - мультипликативно независимые алгебраические числа. Поэтому элементы $\Delta_{2}$ являются вершинами некоторого куба в $\Gamma_{\Delta_{2}} \otimes \mathbb{Q}$. Очевидно, что никакие три такие вершины не лежат на одной аффинной прямой в $\Gamma_{\Delta_{2}} \otimes \mathbb{Q}$.

Предположим, что $S_{2}-$ простая группа типа $B_{m}(m \geqslant 2)$. Тогда в обозначениях Н. Бурбаки

$$
\begin{aligned}
\omega_{m} & =\left(\varepsilon_{1}+\cdots+\varepsilon_{m}\right) / 2, \\
\operatorname{ch} E\left(\omega_{m}\right) & =\sum_{a_{i} \in\{ \pm 1\}} e^{a_{1} \varepsilon_{1} / 2+\cdots+a_{m} \varepsilon_{m} / 2}
\end{aligned}
$$

$\left[4\right.$, гл. 6 , п. 4.5], поэтому можно считать, что $\Delta_{2}=\left\{\alpha_{1}^{a_{1}} \ldots \alpha_{m}^{a_{m}} \mid a_{j} \in\{ \pm 1\}\right\}$, где $\alpha_{1}, \ldots, \alpha_{m}$ - мультипликативно независимые алгебраические числа. Поэтому элементы $\Delta_{2}$ являются вершинами некоторого куба в $\Gamma_{\Delta_{2}} \otimes \mathbb{Q}$, и никакие три такие вершины не лежат на одной аффинной прямой в $\Gamma_{\Delta_{2}} \otimes \mathbb{Q}$.

Предположим, что $S_{2}-$ простая группа типа $C_{m}(m \geqslant 2)$. Тогда

$$
\operatorname{ch} E\left(\omega_{1}\right)=\sum_{\substack{a_{i} \in\{ \pm 1\} \\ i \in\{1, \ldots, m\}}} e^{a_{i} \varepsilon_{i}}
$$

[4, гл. 6, п. 4.6]. Поэтому можно считать, что $\Delta_{2}=\left\{\alpha_{j}^{a_{j}} \mid a_{j} \in\{ \pm 1\}, j \in\right.$ $\{1, \ldots, m\}\}$, где $\alpha_{1}, \ldots, \alpha_{m}$ - мультипликативно независимые алгебраические числа. Поэтому элементы $\Delta_{2}$ являются вершинами некоторого куба в $\Gamma_{\Delta_{2}} \otimes \mathbb{Q}$, и никакие три такие вершины не лежат на одной аффинной прямой в $\Gamma_{\Delta_{2}} \otimes \mathbb{Q}$.

Предположим, что $S_{2}-$ простая группа типа $D_{m}(m \geqslant 3)$. Тогда

$$
\begin{aligned}
\operatorname{ch} E\left(\omega_{1}\right) & =\sum_{\substack{a_{i} \in\{ \pm 1\} \\
i \in\{1, \ldots, m\}}} e^{a_{i} \varepsilon_{i}}, \\
\operatorname{ch} E\left(\omega_{m-1}\right) & \sum_{\substack{a_{i} \in\{ \pm 1\} \\
\operatorname{Card}\left(\left\{i \mid a_{i}=-1\right\}\right)=1(\bmod 2)}} e^{a_{1} \varepsilon_{1} / 2+\cdots+a_{m} \varepsilon_{m} / 2}, \\
\operatorname{ch} E\left(\omega_{m}\right) & \sum_{\substack{a_{i} \in\{ \pm 1\} \\
\operatorname{Card}\left(\left\{i \mid a_{i}=-1\right\}\right)=0(\bmod 2)}}^{a^{a_{1} / 2+\cdots+a_{m} \varepsilon_{m} / 2}}
\end{aligned}
$$

$[4$, гл. 6, п. 4.8$]$. Поэтому можно считать, что

$$
\Delta_{2}=\left\{\alpha_{j}^{a_{j}} \mid a_{j} \in\{ \pm 1\}, j \in\{1, \ldots, m\}\right\}
$$

или

$$
\Delta_{2}=\left\{\alpha_{1}^{a_{1}} \ldots \alpha_{m}^{a_{m}} \mid a_{j} \in\{ \pm 1\}, \operatorname{Card}\left(\left\{j \mid a_{j}=-1\right\}\right)=1 \quad(\bmod 2)\right\}
$$


или

$$
\Delta_{2}=\left\{\alpha_{1}^{a_{1}} \ldots \alpha_{m}^{a_{m}}\left(a_{j} \in\{ \pm 1\}, \operatorname{Card}\left(\left\{j \mid a_{j}=-1\right\}\right)=0 \quad(\bmod 2)\right\},\right.
$$

где $\alpha_{1}, \ldots, \alpha_{m}$ - мультипликативно независимые алгебраические числа. Поэтому в любом случае элементы $\Delta_{2}$ являются вершинами некоторого куба в $\Gamma_{\Delta_{2}} \otimes \mathbb{Q}$, и никакие три такие вершины не лежат на одной абффинной прямой в $\Gamma_{\Delta_{2}} \otimes \mathbb{Q}$. Лемма доказана.

2.8. ЛЕмМА. Если $x, y, z \in \Delta-$-три различные точки, лежсащие на одной аффинной прямой $L \subset \Gamma_{v} \otimes \mathbb{Q}$, то $x=\lambda \cdot \delta_{1}^{\prime} \cdot \delta_{2}, \quad y=\lambda \cdot \delta_{1} \cdot \delta_{2}, \quad z=\lambda \cdot \delta_{1}^{\prime \prime} \cdot \delta_{2}$, әде $\delta_{1}^{\prime}, \delta_{1}, \delta_{1}^{\prime \prime} \in \Delta_{1} u \delta_{2} \in \Delta_{2}$.

ДокАЗАТЕЛЬСтво. Согласно (2.4.6) мы можем рассматривать каноническую проекцию

$$
\operatorname{pr}_{\Delta_{2}}: \Gamma_{v} \otimes \mathbb{Q}=\left(\Gamma_{\{\lambda\}} \otimes \mathbb{Q}\right) \times\left(\Gamma_{\Delta_{1}} \otimes \mathbb{Q}\right) \times\left(\Gamma_{\Delta_{2}} \otimes \mathbb{Q}\right) \rightarrow \Gamma_{\Delta_{2}} \otimes \mathbb{Q}
$$

Если $x, y, z$ - различные элементы $\Delta$, лежашие на афффинной прямой $L$, то $\operatorname{pr}_{\Delta_{2}}(x)$, $\operatorname{pr}_{\Delta_{2}}(y), \operatorname{pr}_{\Delta_{2}}(z)$ лежат на $\operatorname{pr}_{\Delta_{2}}(L)$. Заметим, что если $y$ расположен между $x$ и $z$, то $\operatorname{pr}_{\Delta_{2}}(y)$ расположена между $\operatorname{pr}_{\Delta_{2}}(x)$ и $\operatorname{pr}_{\Delta_{2}}(z)$. Если $\operatorname{pr}_{\Delta_{2}}(L)$ - прямая, то $\operatorname{pr}_{\Delta_{2}}(x), \operatorname{pr}_{\Delta_{2}}(y), \operatorname{pr}_{\Delta_{2}}(z)$ являются различными элементами $\operatorname{pr}_{\Delta_{2}}(\Delta)=\Delta_{2}$, лежащими на аффинной прямой $\operatorname{pr}_{\Delta_{2}}(L)$ вопреки лемме 2.7. Следовательно, $\operatorname{pr}_{\Delta_{2}}(L)$ - единственная точка. Лемма доказана.

\section{§ 3. Корни, веса и собственные числа Фробениуса}

3.1. Мы начинаем доказательство теоремы 0.6 .

Пусть $R$ - система корней $\operatorname{Lie} \operatorname{Im}\left(\rho_{l}\right) \otimes \overline{\mathbb{Q}}_{l}$ относительно подалгебры Картана $h$. Мы обозначаем через $():, h^{*} \times h \rightarrow \overline{\mathbb{Q}}_{l}$ тавтологическое спаривание. Пусть $R^{V}-$ дуальная система корней. Множества $R$ и $R^{V}$ инвариантны относительно естественного действия группы Вейля $W$. Мы определяем решетку весов $P(R)$ в $h^{*}$ :

$$
P(R)=\left\{\lambda \in h^{*} \mid\left(\lambda, \alpha^{V}\right) \in \mathbb{Z} \quad \forall \alpha \in R\right\} .
$$

3.2. Пусть $\Omega\left(V_{l} \otimes \overline{\mathbb{Q}}_{l}\right)$ - множество весов $h \subset \operatorname{Lie} \operatorname{Im}\left(\rho_{l}\right) \otimes \overline{\mathbb{Q}}_{l}$. Мы имеем $\Omega\left(V_{l} \otimes \overline{\mathbb{Q}}_{l}\right) \subset P(R) \subset h^{*}$. Множество $\Omega\left(V_{l} \otimes \overline{\mathbb{Q}}_{l}\right)$ является $W$-инвариантным и $R$-насышенным [4, гл. $8, \S 7$, предложения 3,4$]$. Это означает, что для веса $\lambda \in$ $\Omega\left(V_{l} \otimes \overline{\mathbb{Q}}_{l}\right)$ и такого корня $\alpha \in R$, что $m=\left(\lambda, \alpha^{V}\right)>0$, множество $\Omega\left(V_{l} \otimes \overline{\mathbb{Q}}_{l}\right)$ содержит арифметическую прогрессию $\lambda, \lambda-\alpha, \ldots, \lambda-m \alpha$ длины $m$. Если $m=$ $\left(\lambda, \alpha^{V}\right)<0$, то $\Omega\left(V_{l} \otimes \overline{\mathbb{Q}}_{l}\right)$ содержит арифметическую прогрессию $\lambda, \lambda+\alpha, \ldots, \lambda+$ $(-m) \alpha$ длины $(-m)$.

3.3. Напомним, что ненулевой вес $\lambda \in P(R)$ называется микровесом тогда и только тогда, когда $\left(\lambda, \alpha^{V}\right) \in\{0,1,-1\}$ для всех $\alpha \in R[4$, гл. $8, \S 7$, п. 3].

Если каноническое $l$-адическое представление $\rho_{l}$ не определяется микровесами, то $\Omega\left(V_{l} \otimes \overline{\mathbb{Q}}_{l}\right)$ содержит арифметическую прогрессию типа $\lambda, \lambda-\alpha, \lambda-2 \alpha$ или $\lambda, \lambda+\alpha, \lambda+2 \alpha$. В этом случае существуют такие различные элементы $\delta \neq \delta^{\prime} \neq$ $\delta^{\prime \prime} \in \Delta$, что $\delta^{2}=\delta^{\prime} \cdot \delta^{\prime \prime}$ (действительно, если $\lambda, \lambda-\alpha, \lambda-2 \alpha \in \Omega\left(V_{l} \otimes \overline{\mathbb{Q}}_{l}\right)$, то $2 \cdot(\lambda-\alpha)=\lambda+(\lambda-2 \alpha))$. 
3.4. ЗАмечание. Очевидно, что $\delta$ - середина отрезка $\left[\delta^{\prime}, \delta^{\prime \prime}\right] \subset \Gamma_{v} \otimes \mathbb{Q}$. Поэтому $\delta^{\prime}, \delta$ и $\delta^{\prime \prime}$ лежат на одной аффинной прямой $L \subset \Gamma_{v} \otimes \mathbb{Q}$.

3.5. Рассмотрим множество $\Delta^{\text {line }}=\left\{\left(\delta^{\prime}, \delta, \delta^{\prime \prime}\right) \mid \delta \neq \delta^{\prime} \neq \delta^{\prime \prime} \in \Delta, \delta^{2}=\right.$ $\left.\delta^{\prime} \cdot \delta^{\prime \prime}\right\}$. Очевидно, $\Delta^{\text {line }} \subset \Delta \times \Delta \times \Delta$ инвариантно относительно действия групшы Галуа $\operatorname{Gal}(\overline{\mathbb{Q}} / \mathbb{Q})$, так как для любого $\sigma \in \operatorname{Gal}(\overline{\mathbb{Q}} / \mathbb{Q})$ мы имеем $(\sigma(\delta))^{2}=\sigma\left(\delta^{2}\right)=$ $\sigma\left(\delta^{\prime}\right) \cdot \sigma\left(\delta^{\prime \prime}\right)$.

Последнее соотношение показывает, что множество

$$
B=\left\{\delta^{\prime} / \delta, \delta^{\prime \prime} / \delta=\left(\delta^{\prime} / \delta\right)^{-1} \mid\left(\delta^{\prime}, \delta, \delta^{\prime \prime}\right) \in \Delta^{\text {line }}\right\} \subset \Gamma_{v}
$$

инвариантно относительно естественного действия $\mathrm{Gal}(\overline{\mathbb{Q}} / \mathbb{Q})$.

3.6. Пусть $\left(\delta^{\prime}, \delta, \delta^{\prime \prime}\right) \in \Delta^{\text {line }}$. Согласно лемме 2.8 мы имеем следующие разложения:

$$
\delta^{\prime}=\lambda \cdot \delta_{1}^{\prime} \cdot \delta_{2}, \quad \delta=\lambda \cdot \delta_{1} \cdot \delta_{2}, \quad \delta^{\prime \prime}=\lambda \cdot \delta_{1}^{\prime \prime} \cdot \delta_{2},
$$

где $\delta_{1}^{\prime}, \delta_{1}, \delta_{1}^{\prime \prime} \in \Delta_{1}, \delta_{2} \in \Delta_{2}$.

Пусть $b=\delta^{\prime} / \delta \in B$. Тогда $b=\delta_{1}^{\prime} / \delta_{1}$, и мы получаем из (3.6.1) следуюшие разложения: $\delta^{\prime}=\lambda \cdot b \cdot \delta_{1} \cdot \delta_{2}, \delta=\lambda \cdot \delta_{1} \cdot \delta_{2}, \delta^{\prime \prime}=\lambda \cdot b^{-1} \cdot \delta_{1} \cdot \delta_{2}$. Поскольку $b \cdot \delta_{1}=\delta_{1}^{\prime} \in \Delta_{1}$ и $\delta_{1}^{-1} \in \Delta_{1}$, мы имеем соотношение $b \in \Delta_{1} \cdot \Delta_{1}$. Поэтому $B \subset \Delta_{1} \cdot \Delta_{1}$. С другой стороны, $B \neq\{1\}$, поэтому имеется нетривиальное $\mathrm{Gal}(\overline{\mathbb{Q}} / \mathbb{Q})$-инвариантное подмножество $B \subset \Delta_{1} \cdot \Delta_{1}$, что противоречит теореме 2.5 .

Следовательно, $\rho_{l}$ определено микровесами. Простой фактор типа $E_{6}$ не может появиться, потому что $E\left(\omega_{1,6}\right)$ не является симплектическим или ортогональным представлением [4, гл. 8 , таблица 1]. Мы исключим случай простого фактора типа $E_{7}$ в следуюшем параграфе.

\section{§ 4. Исключение типа $E_{7}$}

4.1. В обозначениях Н. Бурбаки $[4$, гл. $6, \S 4]$ простая алгебра Ли типа $E_{7}$ имеет следующую систему корней:

$$
\begin{aligned}
R=\{ & \pm \varepsilon_{i} \pm \varepsilon_{j} \quad(1 \leqslant i<j \leqslant 6), \pm\left(\varepsilon_{7}-\varepsilon_{8}\right) \\
& \left. \pm \frac{1}{2} \cdot\left(\varepsilon_{7}-\varepsilon_{8}+\sum_{i=1}^{6}(-1)^{\nu(i)} \varepsilon_{i}\right)\left(\sum_{i=1}^{6} \nu(i)=1(\bmod 2)\right)\right\}
\end{aligned}
$$

Базой системы корней является следующее множество:

$$
\begin{aligned}
\left\{\alpha_{1}\right. & =\frac{1}{2}\left(\varepsilon_{1}+\varepsilon_{8}\right)-\frac{1}{2}\left(\varepsilon_{2}+\varepsilon_{3}+\varepsilon_{4}+\varepsilon_{5}+\varepsilon_{6}+\varepsilon_{7}\right) ; \\
\alpha_{2} & =\varepsilon_{1}+\varepsilon_{2} ; \quad \alpha_{3}=\varepsilon_{2}-\varepsilon_{1} ; \quad \alpha_{4}=\varepsilon_{3}-\varepsilon_{2} ; \\
\alpha_{5} & \left.=\varepsilon_{4}-\varepsilon_{3} ; \quad \alpha_{6}=\varepsilon_{5}-\varepsilon_{4} ; \quad \alpha_{7}=\varepsilon_{6}-\varepsilon_{5}\right\} .
\end{aligned}
$$


Для данного $\alpha \in R$ рассмотрим отражение

$$
S_{\alpha}(x)=x-2 \cdot \frac{(x, \alpha)}{(\alpha, \alpha)} \cdot \alpha .
$$

Поскольку $(\alpha, \alpha)=2$ для всех $\alpha \in R$, то мы получаем формулу $S_{\alpha}(x)=x-(x, \alpha) \cdot \alpha$. Эти отражения $S_{\alpha}(\alpha \in R)$ порождают группу Вейля $W(R)$ порядка $2^{10} \cdot 3^{4} \cdot 5 \cdot 7$.

4.2. Мы хотим найти множество всех весов неприводимого симплектического представления $E\left(\omega_{7}\right)$ степени $56=2^{3} \cdot 7$ [4, гл. 8, таблицы 1,2$]$. Поскольку

$$
\omega_{7}=\varepsilon_{6}+\left(\varepsilon_{8}-\varepsilon_{7}\right) / 2=\left(2 \alpha_{1}+3 \alpha_{2}+4 \alpha_{3}+6 \alpha_{4}+5 \alpha_{5}+4 \alpha_{6}+3 \alpha_{7}\right) / 2
$$

- микровес, множество всех весов $E\left(\omega_{7}\right)$ совпадает с $W(R) \cdot \omega_{7}[4$, гл. $8, \S 7$, предложение 6].

Легко найти матрищу $A=\left(a_{i j}\right)=\left(S_{\alpha_{i}}\left(\alpha_{j}\right)\right)$ :

$$
A=\left(\begin{array}{ccccccc}
-\alpha_{1} & \alpha_{2} & \alpha_{1}+\alpha_{3} & \alpha_{4} & \alpha_{5} & \alpha_{6} & \alpha_{7} \\
\alpha_{1} & -\alpha_{2} & \alpha_{3} & \alpha_{2}+\alpha_{4} & \alpha_{5} & \alpha_{6} & \alpha_{7} \\
\alpha_{1}+\alpha_{3} & \alpha_{2} & -\alpha_{3} & \alpha_{3}+\alpha_{4} & \alpha_{5} & \alpha_{6} & \alpha_{7} \\
\alpha_{1} & \alpha_{2}+\alpha_{4} & \alpha_{3}+\alpha_{4} & -\alpha_{4} & \alpha_{4}+\alpha_{5} & \alpha_{6} & \alpha_{7} \\
\alpha_{1} & \alpha_{2} & \alpha_{3} & \alpha_{4}+\alpha_{5} & -\alpha_{5} & \alpha_{5}+\alpha_{6} & \alpha_{7} \\
\alpha_{1} & \alpha_{2} & \alpha_{3} & \alpha_{4} & \alpha_{5}+\alpha_{6} & -\alpha_{6} & \alpha_{6}+\alpha_{7} \\
\alpha_{1} & \alpha_{2} & \alpha_{3} & \alpha_{4} & \alpha_{5} & \alpha_{6}+\alpha_{7} & -\alpha_{7}
\end{array}\right)
$$

Рассмотрим положительный вес $\varepsilon_{3}+\varepsilon_{6}=\alpha_{2}+\alpha_{3}+2 \alpha_{4}+\alpha_{5}+\alpha_{6}+\alpha_{7}$ и соответствующее отражение $S_{\varepsilon_{3}+\varepsilon_{6}}$. Легко видеть, что

$$
\begin{aligned}
& S_{\varepsilon_{3}+\varepsilon_{6}}\left(\alpha_{1}\right)=\alpha_{1}+\alpha_{2}+\alpha_{3}+2 \alpha_{4}+\alpha_{5}+\alpha_{6}+\alpha_{7}, \\
& S_{\varepsilon_{3}+\varepsilon_{6}}\left(\alpha_{2}\right)=\alpha_{2}, \\
& S_{\varepsilon_{3}+\varepsilon_{6}}\left(\alpha_{3}\right)=\alpha_{3}, \\
& S_{\varepsilon_{3}+\varepsilon_{6}}\left(\alpha_{4}\right)=-\alpha_{2}-\alpha_{3}-\alpha_{4}-\alpha_{5}-\alpha_{6}-\alpha_{7}, \\
& S_{\varepsilon_{3}+\varepsilon_{6}}\left(\alpha_{5}\right)=\alpha_{2}+\alpha_{3}+2 \alpha_{4}+2 \alpha_{5}+\alpha_{6}+\alpha_{7}, \\
& S_{\varepsilon_{3}+\varepsilon_{6}}\left(\alpha_{6}\right)=\alpha_{6}, \\
& S_{\varepsilon_{3}+\varepsilon_{6}}\left(\alpha_{7}\right)=-\alpha_{2}-\alpha_{3}-2 \alpha_{4}-\alpha_{5}-\alpha_{6} .
\end{aligned}
$$

Поэтому мы получаем следуюшие цепочки:

$$
\begin{aligned}
& 2 \cdot \omega_{7}=2 \alpha_{1}+3 \alpha_{2}+4 \alpha_{3}+6 \alpha_{4}+5 \alpha_{5}+4 \alpha_{6}+3 \alpha_{7} \stackrel{S_{\varepsilon_{3}+\varepsilon_{6}}}{\longrightarrow} \\
& 2 \alpha_{1}+\alpha_{2}+2 \alpha_{3}+2 \alpha_{4}+3 \alpha_{5}+2 \alpha_{6}+\alpha_{7} \stackrel{S_{\alpha_{1}}}{\longrightarrow} \\
& \alpha_{2}+2 \alpha_{3}+2 \alpha_{4}+3 \alpha_{5}+2 \alpha_{6}+\alpha_{7} \stackrel{S_{\alpha_{3}}}{\longrightarrow} \\
& \alpha_{2}+2 \alpha_{4}+3 \alpha_{5}+2 \alpha_{6}+\alpha_{7} \stackrel{S_{\alpha_{5}}}{\longrightarrow} \\
& \alpha_{2}+2 \alpha_{4}+\alpha_{5}+2 \alpha_{6}+\alpha_{7} \stackrel{S_{\alpha_{4}}}{\longrightarrow} \\
& \alpha_{2}+\alpha_{5}+2 \alpha_{6}+\alpha_{7} \stackrel{S_{\alpha_{2}}}{\longrightarrow}
\end{aligned}
$$




$$
\begin{aligned}
& -\alpha_{2}+\alpha_{5}+2 \alpha_{6}+\alpha_{7} \stackrel{S_{\alpha_{6}}}{\longrightarrow} \\
& -\alpha_{2}+\alpha_{5}+\alpha_{7} \\
& 2 \alpha_{1}+\alpha_{2}+2 \alpha_{3}+2 \alpha_{4}+3 \alpha_{5}+2 \alpha_{6}+\alpha_{7} \stackrel{S_{\alpha_{4}}}{\longrightarrow} \\
& 2 \alpha_{1}+\alpha_{2}+2 \alpha_{3}+4 \alpha_{4}+3 \alpha_{5}+2 \alpha_{6}+\alpha_{7} \stackrel{S_{\alpha_{2}}}{\longrightarrow} \\
& 2 \alpha_{1}+3 \alpha_{2}+2 \alpha_{3}+4 \alpha_{4}+3 \alpha_{5}+2 \alpha_{6}+\alpha_{7} \stackrel{S_{\alpha_{3}}}{\longrightarrow} \\
& 2 \alpha_{1}+3 \alpha_{2}+4 \alpha_{3}+4 \alpha_{4}+3 \alpha_{5}+2 \alpha_{6}+\alpha_{7} \stackrel{S_{\alpha_{4}}}{\longrightarrow} \\
& 2 \alpha_{1}+3 \alpha_{2}+4 \alpha_{3}+6 \alpha_{4}+3 \alpha_{5}+2 \alpha_{6}+\alpha_{7} \stackrel{S_{\alpha_{5}}}{\longrightarrow} \\
& 2 \alpha_{1}+3 \alpha_{2}+4 \alpha_{3}+6 \alpha_{4}+5 \alpha_{5}+2 \alpha_{6}+\alpha_{7} \stackrel{S_{\alpha_{6}}}{\longrightarrow} \\
& 2 \alpha_{1}+3 \alpha_{2}+4 \alpha_{3}+6 \alpha_{4}+5 \alpha_{5}+4 \alpha_{6}+\alpha_{7} \text {; } \\
& 2 \alpha_{1}+\alpha_{2}+2 \alpha_{3}+2 \alpha_{4}+3 \alpha_{5}+2 \alpha_{6}+\alpha_{7} \stackrel{S_{\alpha_{5}}}{\longrightarrow} \\
& 2 \alpha_{1}+\alpha_{2}+2 \alpha_{3}+2 \alpha_{4}+\alpha_{5}+2 \alpha_{6}+\alpha_{7} \stackrel{S_{\alpha_{6}}}{\longrightarrow} \\
& 2 \alpha_{1}+\alpha_{2}+2 \alpha_{3}+2 \alpha_{4}+\alpha_{5}+\alpha_{7} \stackrel{S_{\alpha_{7}}}{\longrightarrow} \\
& 2 \alpha_{1}+\alpha_{2}+2 \alpha_{3}+2 \alpha_{4}+\alpha_{5}-\alpha_{7} \text {; } \\
& \alpha_{2}+2 \alpha_{3}+2 \alpha_{4}+3 \alpha_{5}+2 \alpha_{6}+\alpha_{7} \stackrel{S_{\alpha_{4}}}{\longrightarrow} \\
& \alpha_{2}+2 \alpha_{3}+4 \alpha_{4}+3 \alpha_{5}+2 \alpha_{6}+\alpha_{7} \stackrel{S_{\alpha_{2}}}{\longrightarrow} \\
& 3 \alpha_{2}+2 \alpha_{3}+4 \alpha_{4}+3 \alpha_{5}+2 \alpha_{6}+\alpha_{7} ; \\
& \alpha_{2}+2 \alpha_{3}+2 \alpha_{4}+3 \alpha_{5}+2 \alpha_{6}+\alpha_{7} \stackrel{S_{\alpha_{5}}}{\longrightarrow} \\
& \alpha_{2}+2 \alpha_{3}+2 \alpha_{4}+\alpha_{5}+2 \alpha_{6}+\alpha_{7} \stackrel{S_{\alpha_{6}}}{\longrightarrow} \\
& \alpha_{2}+2 \alpha_{3}+2 \alpha_{4}+\alpha_{5}+\alpha_{7} \stackrel{S_{\alpha_{3}}}{\longrightarrow} \\
& \alpha_{2}+2 \alpha_{4}+\alpha_{5}+\alpha_{7} \stackrel{S_{\alpha_{4}}}{\longrightarrow} \\
& \alpha_{2}+\alpha_{5}+\alpha_{7} \stackrel{S_{\alpha_{5}}}{\longrightarrow} \\
& \alpha_{2}-\alpha_{5}+\alpha_{7} \\
& 2 \alpha_{1}+\alpha_{2}+2 \alpha_{3}+4 \alpha_{4}+3 \alpha_{5}+2 \alpha_{6}+\alpha_{7} \stackrel{S_{\alpha_{3}}}{\longrightarrow} \\
& 2 \alpha_{1}+\alpha_{2}+4 \alpha_{3}+4 \alpha_{4}+3 \alpha_{5}+2 \alpha_{6}+\alpha_{7} ; \\
& \alpha_{2}+2 \alpha_{3}+2 \alpha_{4}+\alpha_{5}+\alpha_{7} \stackrel{S_{\alpha_{7}}}{\longrightarrow} \\
& \alpha_{2}+2 \alpha_{3}+2 \alpha_{4}+\alpha_{5}-\alpha_{7} \stackrel{S_{\alpha_{3}}}{\longrightarrow} \\
& \alpha_{2}+2 \alpha_{4}+\alpha_{5}-\alpha_{7} \text {; } \\
& \alpha_{2}+\alpha_{5}+\alpha_{7} \stackrel{S_{\alpha_{7}}}{\longrightarrow} \\
& \alpha_{2}+\alpha_{5}-\alpha_{7} \text {. }
\end{aligned}
$$


Продолжение этой процедуры приводит к старым весам или к противоположным весам. Следовательно, множество $\Omega\left(E\left(\omega_{7}\right)\right)$ всех весов $E\left(\omega_{7}\right)$ равно $\frac{1}{2} \Omega \cup$ $\left(-\frac{1}{2} \Omega\right)$, где $\Omega$ - множество всех элементов в цепочках, приведенных выше. В итоге мы получаем следуюший список элементов $\Omega$ :

$$
\begin{aligned}
& 2 \alpha_{1}+3 \alpha_{2}+4 \alpha_{3}+6 \alpha_{4}+5 \alpha_{5}+4 \alpha_{6}+3 \alpha_{7} ; \\
& 2 \alpha_{1}+3 \alpha_{2}+4 \alpha_{3}+6 \alpha_{4}+5 \alpha_{5}+4 \alpha_{6}+\alpha_{7} ; \\
& 2 \alpha_{1}+3 \alpha_{2}+4 \alpha_{3}+6 \alpha_{4}+5 \alpha_{5}+2 \alpha_{6}+\alpha_{7} ; \\
& 2 \alpha_{1}+3 \alpha_{2}+4 \alpha_{3}+6 \alpha_{4}+3 \alpha_{5}+2 \alpha_{6}+\alpha_{7} ; \\
& 2 \alpha_{1}+3 \alpha_{2}+4 \alpha_{3}+4 \alpha_{4}+3 \alpha_{5}+2 \alpha_{6}+\alpha_{7} ; \\
& 2 \alpha_{1}+3 \alpha_{2}+2 \alpha_{3}+4 \alpha_{4}+3 \alpha_{5}+2 \alpha_{6}+\alpha_{7} ; \\
& 2 \alpha_{1}+\alpha_{2}+4 \alpha_{3}+4 \alpha_{4}+3 \alpha_{5}+2 \alpha_{6}+\alpha_{7} ; \\
& 2 \alpha_{1}+\alpha_{2}+2 \alpha_{3}+4 \alpha_{4}+3 \alpha_{5}+2 \alpha_{6}+\alpha_{7} ; \\
& 2 \alpha_{1}+\alpha_{2}+2 \alpha_{3}+2 \alpha_{4}+3 \alpha_{5}+2 \alpha_{6}+\alpha_{7} ; \\
& 2 \alpha_{1}+\alpha_{2}+2 \alpha_{3}+2 \alpha_{4}+\alpha_{5}+2 \alpha_{6}+\alpha_{7} ; \\
& 2 \alpha_{1}+\alpha_{2}+2 \alpha_{3}+2 \alpha_{4}+\alpha_{5} \quad+\alpha_{7} ; \\
& 2 \alpha_{1}+\alpha_{2}+2 \alpha_{3}+2 \alpha_{4}+\alpha_{5} \quad-\alpha_{7} \text {; } \\
& 3 \alpha_{2}+2 \alpha_{3}+4 \alpha_{4}+3 \alpha_{5}+2 \alpha_{6}+\alpha_{7} ; \\
& \alpha_{2}+2 \alpha_{3}+4 \alpha_{4}+3 \alpha_{5}+2 \alpha_{6}+\alpha_{7} ; \\
& \alpha_{2}+2 \alpha_{3}+2 \alpha_{4}+3 \alpha_{5}+2 \alpha_{6}+\alpha_{7} ; \\
& \alpha_{2}+2 \alpha_{3}+2 \alpha_{4}+\alpha_{5}+2 \alpha_{6}+\alpha_{7} ; \\
& \alpha_{2}+2 \alpha_{3}+2 \alpha_{4}+\alpha_{5} \quad+\alpha_{7} \\
& \alpha_{2}+2 \alpha_{3}+2 \alpha_{4}+\alpha_{5} \quad-\alpha_{7} ; \\
& \alpha_{2} \quad+2 \alpha_{4}+3 \alpha_{5}+2 \alpha_{6}+\alpha_{7} ; \\
& \alpha_{2} \quad+2 \alpha_{4}+\alpha_{5}+2 \alpha_{6}+\alpha_{7} \\
& \alpha_{2} \quad+2 \alpha_{4}+\alpha_{5} \quad+\alpha_{7} ; \\
& \begin{array}{lll}
\alpha_{2} & +2 \alpha_{4}+\alpha_{5} & -\alpha_{7} ;
\end{array} \\
& \alpha_{2} \quad+\alpha_{5}+2 \alpha_{6}+\alpha_{7} \\
& \begin{array}{lll}
\alpha_{2} & +\alpha_{5} & +\alpha_{7} ;
\end{array} \\
& \alpha_{2} \quad+\alpha_{5} \quad-\alpha_{7} ; \\
& \begin{array}{lll}
\alpha_{2} & -\alpha_{5} & +\alpha_{7} ;
\end{array} \\
& \alpha_{2}-\alpha_{5}-2 \alpha_{6}-\alpha_{7} ; \\
& \begin{array}{lll}
\alpha_{2} & -\alpha_{5} & -\alpha_{7} .
\end{array}
\end{aligned}
$$

4.3. Пусть $\left(x_{1}, \ldots, x_{7}\right)$ - координаты на $\mathbb{Q} \alpha_{1}+\cdots+\mathbb{Q} \alpha_{7}$ относительно $\left\{\alpha_{1}, \ldots, \alpha_{7}\right\}$. Очевидно, что афффинная гиперплоскость $\left\{x \mid x_{7}=1\right\}$ содержит 27 точек $\Omega \cup(-\Omega)$ и аффинная гиперплоскость $\left\{x \mid x_{7}=-1\right\}$ содержит 27 точек 
$\Omega \cup(-\Omega)$. Следовательно, имеется разбиение

$$
\begin{aligned}
\Omega \cup(-\Omega)= & \left\{ \pm\left(2 \alpha_{1}+3 \alpha_{2}+4 \alpha_{3}+6 \alpha_{4}+5 \alpha_{5}+4 \alpha_{6}+3 \alpha_{7}\right)\right\} \\
& \cup\left\{x \in \Omega \cup(-\Omega) \mid x_{7}=-1\right\} \cup\left\{x \in \Omega \cup(-\Omega) \mid x_{7}=1\right\} .
\end{aligned}
$$

Так как $W(R)$ действует транзитивно на $\Omega\left(E\left(\omega_{7}\right)\right)=\frac{1}{2}(\Omega \cup(-\Omega))$, мы видим, что для любого веса $\omega \in \Omega\left(E\left(\omega_{7}\right)\right)$ сушествуют параллельные аффинные гиперплоскости $L_{-\omega}, L_{\omega}$ размерности 6 , которые дают разбиение

$$
\Omega\left(E\left(\omega_{7}\right)\right)=\{ \pm \omega\} \cup\left\{L_{-\omega} \cap \Omega\left(E\left(\omega_{7}\right)\right)\right\} \cup\left\{L_{\omega} \cap \Omega\left(E\left(\omega_{7}\right)\right)\right\}
$$

где $\operatorname{Card}\left(L_{ \pm \omega} \cap \Omega\left(E\left(\omega_{7}\right)\right)\right)=27$. Эти гиперплоскости разделяют $-\omega$ и $\omega$ так, как показано ниже (где звездочки обозначают элементы $\left.\Omega\left(E\left(\omega_{7}\right)\right)\right)$ :

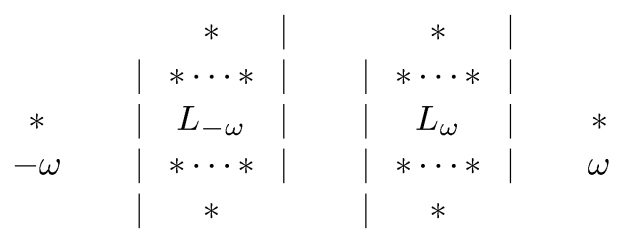

4.4. ОПРЕДЕЛЕНИЕ. Мы Говорим, что подмножество $D \subset \Delta$ имеет конфигурачию типа $E_{7}\left(\omega_{7}\right)$, если и только если существуют такие 7-мерное аффинное подпространство $L \subset \Gamma_{v} \otimes \mathbb{Q}$ и аффинный изоморфизм $f: L \simeq \mathbb{Q} \alpha_{1}+\cdots+\mathbb{Q} \alpha_{7}$, что выполняются следующие условия:

1) $D=L \cap \Delta$

2) $f(D)=\Omega\left(E\left(\omega_{7}\right)\right)$ - система всех весов неприводимого представления $E\left(\omega_{7}\right)$ простой алгебры Ли типа $E_{7}$.

4.5. ЗАмечАниЕ. Если $S_{V_{l}}^{s c} \otimes \overline{\mathbb{Q}}_{l}$ имеет простой фактор типа $E_{7}$, то существует подмножество $D \subset \Delta$ с конфигурацией типа $E_{7}\left(\omega_{7}\right)$.

Действительно, если $\Delta_{1}^{\prime}$ соответствует простому фактору типа $E_{7}$, то, следуя п. 2.4, мы получим разложение $\Delta=\lambda \cdot \Delta_{1}^{\prime} \cdot \Delta_{1}^{\prime \prime} \cdot \Delta_{2}$. Очевидно, что для любых $\delta_{1}^{\prime \prime} \in \Delta_{1}^{\prime \prime}, \delta_{2} \in \Delta_{2}$ аффинное пространство $L=\left(\Gamma_{\Delta_{1}^{\prime}} \otimes \mathbb{Q}\right) \cdot \delta_{1}^{\prime \prime} \cdot \delta_{2}$ содержит подмножество $D=\Delta_{1}^{\prime} \cdot \delta_{1}^{\prime \prime} \cdot \delta_{2}$ с конфигурацией типа $E_{7}\left(\omega_{7}\right)$.

4.6. ЛЕмма. Если $D \subset \Delta$ имеет конфигурацию типа $E_{7}\left(\omega_{7}\right)$, mо $\operatorname{pr}_{\Delta_{2}}(D)$ - единственная точка.

ДоКАЗАТЕЛЬСтво. Обозначим через $\Phi$ композицию следующих отображений:

$$
\mathbb{Q} \alpha_{1}+\cdots+\mathbb{Q} \alpha_{7} \stackrel{f^{-1}}{\longrightarrow} L \stackrel{\mathrm{pr}_{\Delta_{2}}}{\longrightarrow} \Gamma_{\Delta_{2}} \otimes \mathbb{Q}
$$

Отображение $\Phi$ аффинное (возможно, вырожденное). Согласно результатам п. 2.7 $\Phi\left(\Omega\left(E\left(\omega_{7}\right)\right)\right)=\operatorname{pr}_{\Delta_{2}}\left(f^{-1}\left(\Omega\left(E\left(\omega_{7}\right)\right)\right)=\operatorname{pr}_{\Delta_{2}}(D)\right.$ - подмножество множества всех вершин куба в $\Gamma_{\Delta_{2}} \otimes \mathbb{Q}$. Предположим, что это подмножество содержит хотя бы две различные точки. Тогда мы получим из (4.3.1) следуюшую диаграмму (где 
аффинные подпространства $\Phi\left(L_{ \pm \omega}\right)$ параллельны и разделяют вершины $\Phi(-\omega)$ и $\Phi(\omega))$ :

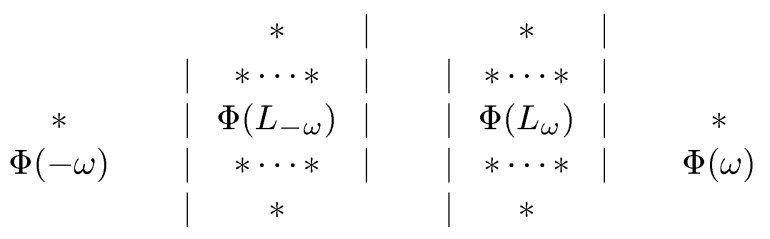

Поскольку все звездочки - вершины куба, мы можем найти такое ребро куба, что вьполняются следуюшие условия:

1) $\Phi(-\omega)$ лежит на этом ребре;

2 ) образ $\Phi(\omega)$ относительно канонической проекции куба на это ребро не равен $\Phi(-\omega)$.

Пусть $\Phi^{\prime}$ - композиция $\Phi$ и проекции куба на рассматриваемое ребро. Мы получаем из (4.6.1) следуюшую диаграмму:

$$
\begin{array}{cccc}
* & * & * & * \\
\Phi^{\prime}(-\omega) & \Phi^{\prime}\left(L_{-\omega}\right) & \Phi^{\prime}\left(L_{\omega}\right) & \Phi^{\prime}(\omega)
\end{array}
$$

Следовательно, мы приходим к противоречию, так как не существует "промежуточных" вершин между двумя различными вершинами куба, лежашими на одном ребре. Лемма доказана.

4.7. ЗАмЕчаниЕ. Предположим, что $D \subset \Delta$ имеет конфигурацию типа $E_{7}\left(\omega_{7}\right)$. Тогда для любого элемента $\sigma \in \mathrm{Gal}(\overline{\mathbb{Q}} / \mathbb{Q})$ множество $\sigma(D) \subset \Delta$ имеет конфигурацию типа $E_{7}\left(\omega_{7}\right)$.

Это очевидно, так как $\operatorname{Gal}(\overline{\mathbb{Q}} / \mathbb{Q})$ действует на $\Gamma_{v} \otimes \mathbb{Q}$ как группа аффинных преобразований.

4.8. Рассмотрим множество

$\Delta^{E_{7}}=\left\{\left(\delta^{\prime}, \delta^{\prime \prime}, \ldots, \delta^{(56)}\right) \in \Delta \times \cdots \times \Delta=\Delta^{56} \mid \delta^{\prime}, \delta^{\prime \prime}, \ldots, \delta^{(56)}-\right.$ различные точки из $\Delta$ и множество $\left\{\delta^{\prime}, \delta^{\prime \prime}, \ldots, \delta^{(56)}\right\}$ имеет конфигурацию типа $\left.E_{7}\left(\omega_{7}\right)\right\}$. В силу замечания 4.7 это множество инвариантно относительно действия группы Галуа $\operatorname{Gal}(\overline{\mathbb{Q}} / \mathbb{Q})$. Следовательно, множество

$$
\left.B=\left\{1, \delta^{\prime \prime} / \delta^{\prime}, \ldots, \delta^{(56)} / \delta^{\prime}, \delta^{\prime} / \delta^{\prime \prime}, \ldots, \delta^{(56)} / \delta^{\prime \prime}, \ldots\right\} \mid\left(\delta^{\prime}, \ldots, \delta^{(56)}\right) \in \Delta^{E_{7}}\right\}
$$

инвариантно относительно естественного действия $\mathrm{Gal}(\overline{\mathbb{Q}} / \mathbb{Q})$. Согласно лемме 4.6 мы имеем следуюшие разложения:

$$
\delta^{\prime}=\lambda \cdot \delta_{1}^{\prime} \cdot \delta_{2}, \quad \delta^{\prime \prime}=\lambda \cdot \delta_{1}^{\prime \prime} \cdot \delta_{2}, \quad \ldots, \quad \delta^{(56)}=\lambda \cdot \delta_{1}^{(56)} \cdot \delta_{2},
$$

где $\delta_{1}^{\prime}, \delta_{1}^{\prime \prime}, \ldots, \delta_{1}^{(56)} \in \Delta_{1}, \delta_{2} \in \Delta_{2}$. Поэтому

$$
\left.B=\left\{1, \delta_{1}^{\prime \prime} / \delta_{1}^{\prime}, \ldots, \delta_{1}^{(56)} / \delta_{1}^{\prime}, \delta_{1}^{\prime} / \delta_{1}^{\prime \prime}, \ldots, \delta_{1}^{(56)} / \delta_{1}^{\prime \prime}, \ldots\right\} \mid\left(\delta^{\prime}, \ldots, \delta^{(56)}\right) \in \Delta^{E_{7}}\right\}
$$

является $\operatorname{Gal}(\overline{\mathbb{Q}} / \mathbb{Q})$-инвариантным подмножеством $\Delta_{1} \cdot \Delta_{1}^{-1}=\Delta_{1} \cdot \Delta_{1}$, и $B \neq\{1\}$ вопреки теореме 2.5. Следовательно, простой фактор типа $E_{7}$ не может появиться. Теорема 0.6 доказана. 


\section{§5. Сушествование обыкновенной редукции поверхности типа К3: сведе́ние задачи к случаю абелева многообразия}

5.1. В дальнейшем мы считаем, что $S$ - гладкая проективная поверхность типа $\mathrm{K} 3$ над числовым полем $k \subset \mathbb{C}$ с полупростой группой Ходжа $\operatorname{Hg}\left(S \otimes_{k} \mathbb{C}\right)$. Пусть $\eta \in H^{2}\left(S \otimes_{k} \mathbb{C}, \mathbb{Z}(1)\right)$ - класс обильного дивизора на $S . \cup$-произведение

$$
H^{2}\left(S \otimes_{k} \mathbb{C}, \mathbb{Z}\right) \otimes H^{2}\left(S \otimes_{k} \mathbb{C}, \mathbb{Z}\right) \rightarrow H^{4}\left(S \otimes_{k} \mathbb{C}, \mathbb{Z}\right) \widetilde{\longrightarrow}(-2)
$$

индуцирует $\psi$ :

$$
P^{2}\left(S \otimes_{k} \mathbb{C}, \mathbb{Z}(1)\right) \otimes P^{2}\left(S \otimes_{k} \mathbb{C}, \mathbb{Z}(1)\right) \rightarrow \mathbb{Z},
$$

где $P^{2}\left(S \otimes_{k} \mathbb{C}, \mathbb{Z}(1)\right)$ - примитивная решетка. Форма $\psi$ имеет сигнатуру $(2+, 19-)$. Пусть $C^{+}\left(P^{2}\left(S \otimes_{k} \mathbb{C}, \mathbb{Z}(1)\right), \psi\right)$ - четная алгебра Клиффорда, ассоциированная с формой $\psi$. Из результатов Сатаке следует, что естественная $\mathbb{Q}$-структура Ходжа на $P^{2}\left(S \otimes_{k} \mathbb{C}, \mathbb{Q}(1)\right)$ типа $(-1,1)+(0,0)+(1,-1)$ определяет $\mathbb{Q}$-структуру Ходжа типа $(1,0)+(0,1)$ на $C^{+}\left(P^{2}\left(S \otimes_{k} \mathbb{C}, \mathbb{Q}(1)\right), \psi\right)$ и абелево многообразие

$$
J_{\mathbb{C}}=C^{+}\left(P^{2}\left(S \otimes_{k} \mathbb{C}, \mathbb{Q}(1)\right), \psi\right) \otimes_{\mathbb{Q}} \mathbb{R} / C^{+}\left(P^{2}\left(S \otimes_{k} \mathbb{C}, \mathbb{Z}(1)\right), \psi\right)
$$

размерности $2^{19}$ над полем комплексных чисел $\mathbb{C}[9$, пा. 4.1-4.5]. Более того, заменив $k$ некоторым конечным алгебраическим расширением, мы можем считать, что $J_{\mathbb{C}}=J \otimes_{k} \mathbb{C}$ для некоторого абелева многообразия $J$ над $k$ и существует такая подалгебра $C^{+}{ }_{\text {в }} \operatorname{End}(J)$, что $\mathbb{Z}_{l}[\operatorname{Gal}(\bar{k} / k)]$-алгебра $\operatorname{End}_{C^{+}} H_{\text {et }}^{1}\left(J \otimes_{k}\right.$ $\left.\bar{k}, \mathbb{Z}_{l}\right)$ изоморфна четной алгебре Клиффорда примитивного квадратичного модуля $P_{\mathrm{et}}^{2}\left(S \otimes_{k} \bar{k}, \mathbb{Z}_{l}(1)\right)$ (с естественным действием группы Галуа) [1, основная лемма 1.7.1]. В частности, мы имеем канонический изоморфизм $\operatorname{Gal}(\bar{k} / k)$-модулей $\bigoplus \bigwedge^{2 i} P_{\text {et }}^{2}\left(S \otimes_{k} \bar{k}, \mathbb{Q}_{l}(1)\right) \stackrel{\sim}{\rightarrow} \operatorname{End}_{C^{+}} H_{\text {et }}^{1}\left(J \otimes \bar{k}, \mathbb{Q}_{l}\right)[9$, пп. 6.6, 4.4]. Каноническое спаривание $\operatorname{Gal}(\bar{k} / k)$-модулей

$$
\bigwedge^{20} P_{\mathrm{et}}^{2}\left(S \otimes_{k} \bar{k}, \mathbb{Q}_{l}(1)\right) \times P_{\mathrm{et}}^{2}\left(S \otimes_{k} \bar{k}, \mathbb{Q}_{l}(1)\right) \rightarrow \bigwedge^{21} P_{\mathrm{et}}^{2}\left(S \otimes_{k} \bar{k}, \mathbb{Q}_{l}(1)\right)=\mathbb{Q}_{l}
$$

невырождено, поэтому мы имеем морфизмы $\mathrm{Gal}(\bar{k} / k)$-модулей

$$
\begin{aligned}
P_{\mathrm{et}}^{2}(S & \left.\otimes_{k} \bar{k}, \mathbb{Q}_{l}(1)\right) \widetilde{\rightarrow} P_{\mathrm{et}}^{2}\left(S \otimes_{k} \bar{k}, \mathbb{Q}_{l}(1)\right)^{*}=\bigwedge^{20} P_{\mathrm{et}}^{2}\left(S \otimes_{k} \bar{k}, \mathbb{Q}_{l}(1)\right) \\
& \subset \operatorname{End}_{C^{+}} H_{\mathrm{et}}^{1}\left(J \otimes \bar{k}, \mathbb{Q}_{l}\right) \subset \operatorname{End}_{\mathbb{Q}_{l}} H_{\mathrm{et}}^{1}\left(J \otimes \bar{k}, \mathbb{Q}_{l}\right) \\
& =H_{\mathrm{et}}^{1}\left(J \otimes \bar{k}, \mathbb{Q}_{l}\right) \otimes_{\mathbb{Q}_{l}} H_{\mathrm{et}}^{1}\left(J \otimes \bar{k}, \mathbb{Q}_{l}\right)^{*} \stackrel{\rightarrow}{\rightarrow} H_{\mathrm{et}}^{1}\left(J \otimes \bar{k}, \mathbb{Q}_{l}\right) \otimes_{\mathbb{Q}_{l}} H_{\mathrm{et}}^{1}\left(J \otimes \bar{k}, \mathbb{Q}_{l}\right)(1) .
\end{aligned}
$$

Следовательно, существует вложение $\operatorname{Gal}(\bar{k} / k)$-модулей

$$
P_{\mathrm{et}}^{2}\left(S \otimes_{k} \bar{k}, \mathbb{Q}_{l}\right) \subset H_{\mathrm{et}}^{1}\left(J \otimes \bar{k}, \mathbb{Q}_{l}\right) \otimes_{\mathbb{Q}_{l}} H_{\mathrm{et}}^{1}\left(J \otimes \bar{k}, \mathbb{Q}_{l}\right),
$$

поэтому любое собственное число $\delta$ линейного оператора $\rho_{l}^{S}\left(F_{\bar{v}}^{-1}\right)$ является произведением двух собственных чисел линейного оператора $\rho_{l}^{J}\left(F_{\bar{v}}^{-1}\right)$, где $\rho_{l}^{J}$ : 
$\operatorname{Gal}(\bar{k} / k) \rightarrow \operatorname{GL}\left(H_{\mathrm{et}}^{1}\left(J \otimes \bar{k}, \mathbb{Q}_{l}\right)\right)$ - естественное $l$-адическое представление. Значит, мы должны доказать, что если $k$ - достаточно большое, то плотность Дирихле множества $\{v \mid v$ - неархимедова точка $k$, в которой $J$ имеет обыкновенную редукцию $\}$ равна 1 .

5.2. С помощью аналогичных аргументов мы доказываем существование вложения $\mathbb{Q}$-структур Ходжа $P_{\text {et }}^{2}\left(S \otimes_{k} \mathbb{C}, \mathbb{Q}\right) \subset H^{1}\left(J \otimes_{k} \mathbb{C}, \mathbb{Q}\right) \otimes_{\mathbb{Q}} H^{1}\left(J \otimes_{k} \mathbb{C}, \mathbb{Q}\right)$, которое определяет изогению $\mathrm{MT}\left(J \otimes_{k} \mathbb{C}\right) \rightarrow \operatorname{MT}\left(S \otimes_{k} \mathbb{C}\right)$. В силу (0.9.1) сушествует канонический изоморфизм алгебр Ли

$$
\operatorname{Lie} \operatorname{Im}\left(\rho_{l}^{J}\right) \widetilde{\rightarrow} \operatorname{Lie}\left[\operatorname{MT}\left(J \otimes_{k} \mathbb{C}\right)\left(\mathbb{Q}_{l}\right)\right] .
$$

В частности, гипотеза Серра-Мамфорда-Тэйта [11] верна для $J$.

5.3. По теореме Лефшеца сушествует канонический изоморфизм

$$
\mathrm{NS}\left(S \otimes_{k} \mathbb{C}\right) \widetilde{\rightarrow}\left[H^{2}\left(S \otimes_{k} \mathbb{C}, \mathbb{Z}\right) \cap H^{1,1}\left(S \otimes_{k} \mathbb{C}, \mathbb{C}\right)\right](1) .
$$

Обозначим через $V_{\mathbb{Q}}(1)$ пространство трансцендентных классов когомологий (ортогональное дополнение $\mathrm{NS}\left(S \otimes_{k} \mathbb{C}\right) \otimes \mathbb{Q}$ относительно $\cup$-произведения), и пусть $d=\operatorname{dim}_{\mathbb{Q}} V_{\mathbb{Q}}(1)=22-\operatorname{rank} \mathrm{NS}\left(S \otimes_{k} \mathbb{C}\right)$. Напомним, что форма $\psi$ имеет сигнатуру $(2+, 19-)$. По теореме об индексе [12, лекция 18$]$ ограничение $\psi_{0}$ формы $\psi$ на $V_{\mathbb{Q}}(1)$ имеет сигнатуру $(2+,(d-2)-)$. Пусть $C^{+}\left(V_{\mathbb{Q}}(1), \psi_{0}\right)$ - четная алгебра Клиффорда, ассоциированная с формой $\psi_{0}$. Из результатов Сатаке следует, что естественная $\mathbb{Q}$-структура Ходжа на $V_{\mathbb{Q}}(1)$ типа $(-1,1)+(0,0)+(1,-1)$ определяет $\mathbb{Q}$-структуру Ходжа типа $(1,0)+(0,1)$ на $C^{+}\left(V_{\mathbb{Q}}(1), \psi_{0}\right)$ и абелево многообразие

$$
J_{\mathbb{C}}^{\mathrm{NS}}=C^{+}\left(V_{\mathbb{Q}}(1), \psi_{0}\right) \otimes_{\mathbb{Q}} \mathbb{R} / C^{+}\left(V_{\mathbb{Z}}(1), \psi_{0}\right)
$$

размерности $2^{d-2}$ надполем комплексных чисел $\mathbb{C}[9$, пп. 4.1-4.5]. Более того, заменив $k$ некоторым конечным алгебраическим расширением, мы можем считать, что $J_{\mathbb{C}}^{\mathrm{NS}}=J^{\mathrm{NS}} \otimes_{k} \mathbb{C}$ для некоторого абелева многообразия $J^{\mathrm{NS}}$ над $k$ и сушествует такая подалгебра $C^{+}{ }_{\text {в }} \operatorname{End}\left(J^{\mathrm{NS}}\right)$, что $\mathbb{Z}_{l}[\mathrm{Gal}(\bar{k} / k)]$-алгебра $\operatorname{End}_{C^{+}} H_{\mathrm{et}}^{1}\left(J^{\mathrm{NS}} \otimes_{k} \bar{k}, \mathbb{Z}_{l}\right)$ изоморфна четной алгебре Клиффорда $C^{+}\left(V_{\mathbb{Z}_{l}}(1), \psi_{0 l}\right)$, где $V_{\mathbb{Z}_{l}}(1)$ - ортогональное дополнение $\mathrm{NS}(S \otimes \bar{k}) \otimes \mathbb{Z}_{l} \subset H_{\mathrm{et}}^{2}\left(S \otimes \bar{k}, \mathbb{Z}_{l}(1)\right)$ (с естественным действием группы Галуа). Действительно, можно считать, что $J$ изогенно степени $J^{\mathrm{NS}}$ над $k[1$, варианты $4.1 .3,4.1 .5]$. В силу [1, основная лемма 1.7.1] мы имеем канонический изоморфизм $\operatorname{Gal}(\bar{k} / k)$-модулей

$$
\bigoplus \bigwedge^{2 i} V_{\mathbb{Q}_{l}}(1) \stackrel{\rightarrow}{\rightarrow} \operatorname{End}_{C^{+}} H_{\mathrm{et}}^{1}\left(J^{\mathrm{NS}} \otimes \bar{k}, \mathbb{Q}_{l}\right)
$$

$[9$, пाп. 6.6, 4.4].

5.4. Приведем описание группы Ходжа $\operatorname{Hg}\left(S \otimes_{k} \mathbb{C}\right)$, принадлежашее Ю. Г. Зархину [34]:

$\mathbb{Q}$-алгебра $K=\operatorname{End}_{\mathrm{Hg}\left(S \otimes_{k} \mathbb{C}\right)} V_{\mathbb{Q}}$ является вполне вещественным полем алгебраических чисел или чисто мнимым квадратичным расширением вполне вещественного поля $K_{0}$. В первом случае $\mathrm{Hg}\left(S \otimes_{k} \mathbb{C}\right)=R_{K / \mathbb{Q}} \mathrm{SO}\left(V_{\mathbb{Q}}, \Phi\right)$, где 
$V_{\mathbb{Q}}$ рассматривается как векторное простанство над $K$, и форма $\Phi: V_{\mathbb{Q}} \times$ $V_{\mathbb{Q}} \rightarrow K,(x, y) \rightarrow \alpha$, определена соотношением $\psi_{0}(e x, y)=\operatorname{Tr}_{K / \mathbb{Q}}(e \alpha)$ для всехе $\in K$. Во втором случае $\operatorname{Hg}\left(S \otimes_{k} \mathbb{C}\right)=R_{K_{0} / \mathbb{Q}} \mathrm{U}\left(V_{\mathbb{Q}}, \Phi\right)$, где $\mathrm{U}\left(V_{\mathbb{Q}}, \Phi\right)$ унитарная группа над $K_{0}$, ассоциированная с $K$-векторным пространством $V_{\mathbb{Q}}$ и формой $\Phi$ на нем (линейной по одному аргументу и полулинейной по другому).

В дальнейшем $e=[K: \mathbb{Q}], d=\operatorname{dim}_{\mathbb{Q}} V_{\mathbb{Q}}$.

\section{§ 6. Существование обыкновенной редукции абелева многообразия Куги-Сатаке}

6.1. Предположим, что $k$ - достаточно большое числовое поле. Для абелева многообразия $J^{\mathrm{NS}}$ рассмотрим естественное $l$-адическое представление $\rho_{l}: \operatorname{Gal}(\bar{k} / k) \rightarrow \mathrm{GL}\left(V_{l}\right)$, где $V_{l}=H_{\mathrm{et}}^{1}\left(J^{\mathrm{NS}} \otimes_{k} \bar{k}, \mathbb{Q}_{l}\right)$. В силу (5.2.1) существует канонический изоморфизм алгебр Ли

$$
\operatorname{Lie} \operatorname{Im}\left(\rho_{l}\right) \stackrel{\longrightarrow}{\rightarrow} \operatorname{Lie}\left[\operatorname{MT}\left(J^{\mathrm{NS}} \otimes_{k} \mathbb{C}\right)\left(\mathbb{Q}_{l}\right)\right] .
$$

Мы хотим доказать, что $\left\{v \mid v\right.$-неархимедова точка $k$, в которой $J^{\mathrm{NS}}$ имеет обыкновенную редукцию $\}$ является множеством плотности 1 по Дирихле.

6.2. В дальнейшем мы предполагаем, что неархимедова точка $v$ и $J=J^{\mathrm{NS}}$ удовлетворяют условиям п. 2.1. Обозначим через $\Delta$ множество всех собственных чисел $\rho_{l}\left(F_{\bar{v}}^{-1}\right)$ (без учета кратностей). Пусть $g_{l}^{s s}$ - полупростая часть редуктивной алгебры Ли $g_{l}=\operatorname{Lie} \operatorname{Im}\left(\rho_{l}\right)=\operatorname{Lie} G_{V_{l}}$.

6.3. Группа Галуа $\operatorname{Gal}(\overline{\mathbb{Q}} / \mathbb{Q})$ действует естественным образом на $\Delta$, и для любой константы $c$ множество

$$
\left\{\eta \in \Delta \cdot \Delta \mid \operatorname{Card}\left(T_{\eta}(\Delta) \cap \Delta\right)=c\right\} \text { является } \operatorname{Gal}(\overline{\mathbb{Q}} / \mathbb{Q}) \text {-инвариантным. (6.3.1) }
$$

6.4. Рассмотрим случай $e=1, d=2 m+1$ - нечетное число. По теореме Ю.Г. Зархина (п. 5.4) $\operatorname{Lie} \operatorname{Hg}\left(S \otimes_{k} \mathbb{C}\right) \otimes \overline{\mathbb{Q}}=\operatorname{Lie} \operatorname{Hg}\left(J^{\mathrm{NS}} \otimes_{k} \mathbb{C}\right) \otimes \overline{\mathbb{Q}}$ - простая алгебра Ли типа $B_{m}(m \geqslant 1)$. В обозначениях [4]

$$
H^{1}\left(J^{\mathrm{NS}} \otimes_{k} \mathbb{C}, \mathbb{Q}\right) \otimes_{\mathbb{Q}} \overline{\mathbb{Q}}=\bigoplus E\left(\omega_{m}\right)
$$

где $E\left(\omega_{m}\right)$ - спинорное представление. В силу (6.1.1) гипотеза Серра-МамфордаТэйта верна для $J^{\mathrm{NS}}$. Поэтому $g_{l}^{s s} \otimes \overline{\mathbb{Q}_{l}}-$ простая алгебра Ли типа $B_{m}$, и мы получаем следуюшее разложение $g_{l}^{s s} \otimes \overline{\mathbb{Q}} l$-модулей:

$$
H_{\mathrm{et}}^{1}\left(J^{\mathrm{NS}} \otimes_{k} \bar{k}, \mathbb{Q}_{l}\right) \otimes_{\mathbb{Q}_{l}} \overline{\mathbb{Q}_{l}}=\bigoplus E\left(\omega_{m}\right)
$$

Дальнейшее является модификацией основной идеи $[14$, п. 2.3].

Описание группы Вейля в $[4$, гл. $6, \S 4$, п. 5$]$ показывает, что

$$
\operatorname{ch} E\left(\omega_{m}\right)=\sum_{a_{i} \in\{ \pm 1\}} e^{a_{1} \varepsilon_{1} / 2+\cdots+a_{m} \varepsilon_{m} / 2}
$$


В силу (2.1.1)-(2.1.6) и (6.4.1) можно считать, что $\Delta=\left\{\lambda \alpha_{1}^{ \pm 1} \alpha_{2}^{ \pm 1} \ldots \alpha_{m}^{ \pm 1}\right\}$, где $\lambda, \alpha_{1}, \ldots, \alpha_{m}$ - мультипликативно независимые алгебраические числа. Известная теорема А. Вейля $\left[13, \S 21\right.$, теорема 4] дает соотношение $\delta \cdot \bar{\delta}=p_{v}$ для любого $\delta \in \Delta$ и для любого комплексного сопряжения на $\overline{\mathbb{Q}}$. Поэтому имеется хорошо определенное комплексное сопряжение на $\Gamma_{v}$. Мультипликативная независимость $\lambda, \alpha_{1}, \ldots, \alpha_{m}$ влечет $\eta=\lambda^{2} \Leftrightarrow \operatorname{Card}\left(T_{\eta}(\Delta) \cap \Delta\right)=\operatorname{Card}(\Delta)$, поэтому (6.3.1) дает соотношение $\lambda^{2} \in \mathbb{Q}$. По теореме Вейля $\lambda^{2}= \pm p_{v}$. Поскольку $\lambda^{2} \in \Gamma_{v}, \delta$. $\bar{\delta}=p_{v} \in \Gamma_{v}$, и $\Gamma_{v}$ не имеет кручения по условию (2.1.5), мы получаем соотношение $\lambda^{2}=p_{v}$. Поэтому для $\delta=\lambda \alpha_{1}^{a_{1}} \ldots \alpha_{m}^{a_{m}}\left(a_{i} \in\{ \pm 1\}\right)$ мы имеем $\bar{\delta}=\lambda \alpha_{1}^{-a_{1}} \ldots \alpha_{m}^{-a_{m}}$.

Пусть $w$ - точка $\overline{\mathbb{Q}}$, лежашая над $p_{v}$ и нормализованная условием $w\left(p_{v}\right)=1$. Она дает отображение из $\overline{\mathbb{Q}}^{\times}$в $\mathbb{Q}$ и, следовательно, отображение $\Gamma_{v} \rightarrow \mathbb{Q}$. На множестве $\Delta w$ принимает значения во множестве $[0,1]$, и чтобы доказать обыкновенность $J_{v}^{\mathrm{NS}}$, мы должны показать, что могут появиться только значения 0 и 1 . Предположим, что она принимает другое значение, и приведем все к противоречию.

Для любых $1 \leqslant j \leqslant m$ рассмотрим алгебраические числа $\beta_{j}^{+}=\lambda^{2} \alpha_{j}^{2} \in \Delta \cdot \Delta$ и $\beta_{j}^{-}=\lambda^{2} \alpha_{j}^{-2} \in \Delta \cdot \Delta$. Мы утверждаем, что наше предположение $w(\delta) \notin\{0,1\}$ для некоторого $\delta \in \Delta$ влечет $w\left(\beta_{j}^{+}\right)>0$ и $w\left(\beta_{j}^{-}\right)>0$ для всех $j$.

Действительно, имеется разбиение $\Delta=\Delta_{j}^{+} \cup \Delta_{j}^{-}$, где $\Delta_{j}^{+}=\left\{\lambda \alpha_{1}^{ \pm 1} \ldots\right.$ $\left.\ldots \alpha_{j} \ldots \alpha_{m}^{ \pm 1}\right\}, \Delta_{j}^{-}=\left\{\lambda \alpha_{1}^{ \pm 1} \ldots \alpha_{j}^{-1} \ldots \alpha_{m}^{ \pm 1}\right\}$. Поэтому $\Delta_{j}^{+} \cap\{\delta, \bar{\delta}\} \neq \varnothing, \Delta_{j}^{-} \cap$ $\{\delta, \bar{\delta}\} \neq \varnothing$. Заметим, что $w(\delta)+w(\bar{\delta})=w\left(p_{v}\right)=1$, поэтому $w(\bar{\delta}) \notin\{0,1\}$. Следовательно, можно считать, что $\delta \in \Delta_{j}^{+}$. Поэтому $\beta_{j}^{+}=\delta \cdot \delta^{\prime}$ для некоторого $\delta^{\prime} \in \Delta$. Следовательно, $w\left(\beta_{j}^{+}\right)=w(\delta)+w\left(\delta^{\prime}\right)>0$. Аналогично мы получаем соотношение $w\left(\beta_{j}^{-}\right)>0$.

Пусть $\eta \in \Delta \cdot \Delta$. Можно считать, что $\eta \in\left\{\lambda^{2}, \lambda^{2} \alpha_{1}^{2}, \lambda^{2} \alpha_{1}^{2} \alpha_{2}^{2}, \ldots, \lambda^{2} \alpha_{1}^{2} \ldots \alpha_{m}^{2}\right\}$. Легко видеть, что для $\delta_{1} \in \Delta$

$$
\begin{aligned}
& T_{\lambda^{2}}\left(\delta_{1}\right) \in \Delta \Leftrightarrow \delta_{1} \in \Delta, \\
& T_{\lambda^{2} \alpha_{1}^{2}}\left(\delta_{1}\right) \in \Delta \Leftrightarrow \delta_{1} \in\left\{\lambda \alpha_{1} \alpha_{2}^{ \pm 1} \ldots \alpha_{m}^{ \pm 1}\right\}, \\
& \ldots \ldots \ldots \ldots \ldots \ldots \ldots \ldots \ldots \ldots \ldots \ldots \ldots \ldots \ldots \ldots \ldots \\
& T_{\lambda^{2} \alpha_{1}^{2} \ldots \alpha_{m}^{2}}\left(\delta_{1}\right) \in \Delta \Leftrightarrow \delta_{1} \in\left\{\lambda \alpha_{1} \alpha_{2} \ldots \alpha_{m}\right\} .
\end{aligned}
$$

Поэтому $\eta \in\left\{\lambda^{2} \alpha_{j}^{ \pm 2} \mid j=1, \ldots, m\right\} \Leftrightarrow \operatorname{Card}\left(T_{\eta}(\Delta) \cap \Delta\right)=2^{m-1}$. Из соотношения (6.3.1) следует, что $\left\{\lambda^{2} \alpha_{j}^{ \pm 2} \mid j=1, \ldots, m\right\}=\left\{\beta_{j}^{+}, \beta_{j}^{-} \mid j=1, \ldots, m\right\}$ является $\operatorname{Gal}(\overline{\mathbb{Q}} / \mathbb{Q})$-инвариантным подмножеством $\Delta \cdot \Delta$. Следовательно, $\beta=\sum_{j=1}^{m}\left(\beta_{j}^{+}+\right.$ $\left.\beta_{j}^{-}\right) \in \mathbb{Z}$ и $w(\beta)>0$. Поэтому $p_{v} \mid \beta, \beta=p_{v} \cdot b_{v}$, где $b_{v} \in \mathbb{Z}$, и $\left|b_{v}\right| \leqslant 2 m$ в силу соотношений $\left|\alpha_{j}\right|=1(j=1, \ldots, m)$.

Мы имеем соотношения (2.5.2) и (2.5.3) в этой новой ситуации. По условию $2.1,1)$ все точки $l^{n}$-кручения в $J^{\mathrm{NS}}(\bar{k})$ рациональны над $k$. Следовательно, $\left.\rho_{l}\right|_{\mathrm{Gal}(\bar{k} / k)}$ тривиально по $\bmod l^{n}$, и поэтому

$$
\beta=p_{v} \cdot b_{v}=2 m \quad\left(\bmod l^{n}\right) .
$$


В силу (2.5.3) мы выводим из (6.4.2), что $b_{v}=2 m\left(\bmod l^{n}\right)$. Ясно, что неравенства $\left|b_{v}\right| \leqslant 2 m<4 m \leqslant\left(2 \cdot \operatorname{dim}_{k} J^{\mathrm{NS}}\right)^{2}<l^{n}$ влекут соотношение $b_{v}=2 m$. Следовательно, $\beta=2 m p_{v}$, и поэтому все $\lambda^{2} \alpha_{j}^{ \pm 2}=p_{v} \alpha_{j}^{ \pm 2}$ равны $p_{v}$, что противоречит мультипликативной независимости $\lambda, \alpha_{1}, \ldots, \alpha_{m}$. Значит, $J_{v}^{\mathrm{NS}}$ - обыкновенное абелево многообразие.

6.5. Рассмотрим случай $e=1, d=2 m$-четное число, $m \geqslant 2$. В силу теоремы Ю.Г. Зархина (п. 5.4) $\operatorname{Lie} \operatorname{Hg}\left(S \otimes_{k} \mathbb{C}\right) \otimes \overline{\mathbb{Q}}=\operatorname{Lie} \operatorname{Hg}\left(J^{\mathrm{NS}} \otimes_{k} \mathbb{C}\right) \otimes \overline{\mathbb{Q}}-$ полупростая алгебра Ли типа $D_{m}(m \geqslant 2)$. Поэтому можно считать, что

$$
H_{\mathrm{et}}^{1}\left(J^{\mathrm{NS}} \otimes_{k} \bar{k}, \mathbb{Q}_{l}\right) \otimes \overline{\mathbb{Q}_{l}}=\bigoplus\left(E\left(\omega_{m-1}\right) \oplus E\left(\omega_{m}\right)\right) .
$$

Описание группы Вейля в $[4$, гл. $6, \S 4$, п. 4.8$]$ показывает, что

$$
\begin{aligned}
\operatorname{ch} E\left(\omega_{m-1}\right)= & \sum_{\substack{a_{i} \in\{ \pm 1\} \\
\operatorname{Card}\left(\left\{i \mid a_{i}=-1\right\}\right)=1(\bmod 2)}} e^{a_{1} \varepsilon_{1} / 2+\cdots+a_{m} \varepsilon_{m} / 2}, \\
\operatorname{ch} E\left(\omega_{m}\right)= & \sum_{\substack{a_{i} \in\{ \pm 1\} \\
\operatorname{Card}\left(\left\{i \mid a_{i}=-1\right\}\right)=0(\bmod 2)}} e^{a_{1} \varepsilon_{1} / 2+\cdots+a_{m} \varepsilon_{m} / 2} .
\end{aligned}
$$

Поэтому из условий п. 6.2 и (6.5.1) следует, что

$$
\begin{aligned}
\Delta= & \left\{\lambda \alpha_{1}^{a_{1}} \ldots \alpha_{m}^{a_{m}} \mid a_{j} \in\{ \pm 1\}, \operatorname{Card}\left(\left\{j \mid a_{j}=-1\right\}\right)=1(\bmod 2)\right\} \\
& \cup\left\{\lambda \alpha_{1}^{a_{1}} \ldots \alpha_{m}^{a_{m}} \mid a_{j} \in\{ \pm 1\}, \operatorname{Card}\left(\left\{j \mid a_{j}=-1\right\}\right)=0(\bmod 2)\right\} \\
= & \left\{\lambda \alpha_{1}^{ \pm 1} \ldots \alpha_{m}^{ \pm 1}\right\},
\end{aligned}
$$

где $\lambda, \alpha_{1}, \ldots, \alpha_{m} \in \overline{\mathbb{Q}}^{\times}$мультипликативно независимы (другими словами, эти числа порождают мультипликативную подгруппу в $\overline{\mathbb{Q}}^{\times}$ранга $\left.m+1\right)$. Поэтому мы можем доказать, что специальный слой $J_{v}^{\mathrm{NS}}$ является обыкновенным, с помошью процедуры п. 6.4 .

6.6. Предположим, что $K$-вполне вешественное поле и $d / e=2 m+1$ - нечетное число. В силу теоремы Ю.Г.Зархина (п. 5.4) $\mathrm{Lie} \mathrm{Hg}\left(J^{\mathrm{NS}} \otimes_{k} \mathbb{C}\right) \otimes \overline{\mathbb{Q}}$ - полупростая алгебра Ли типа $B_{m} \times \cdots \times B_{m}=B_{m}^{e}(m \geqslant 1)$.

В силу теоремы 0.3 каноническое представление $\operatorname{Lie} \operatorname{Hg}\left(J^{\mathrm{NS}} \otimes_{k} \mathbb{C}\right) \otimes_{\mathbb{Q}} \overline{\mathbb{Q}}$ в $H^{1}\left(J^{\mathrm{NS}} \otimes_{k} \mathbb{C}, \mathbb{Q}\right) \otimes_{\mathbb{Q}} \overline{\mathbb{Q}}$ определено микровесами классических алгебр Ли. В силу (6.1.1) каноническое представление $g_{l}^{s s} \otimes \overline{\mathbb{Q}_{l}}$ определено микровесами классических алгебр Ли. Легко видеть, что $H^{1}\left(J^{\mathrm{NS}} \otimes_{k} \mathbb{C}, \mathbb{Q}\right) \otimes_{\mathbb{Q}} \overline{\mathbb{Q}}=\bigoplus E\left(\omega_{m}^{(1)}+\cdots+\omega_{m}^{(e)}\right)$, где индекс $(i)$ показывает, что соответствуюший фундаментальный вес относится к $i$-му фактору [24, формулы (1.7.4), (1.7.5)]. Можно считать, что $\Delta=\left\{\lambda \alpha_{1}^{ \pm 1} \alpha_{2}^{ \pm 1} \ldots \alpha_{m e}^{ \pm 1}\right\}$, где $\lambda, \alpha_{1}, \ldots, \alpha_{m e}-$ мультипликативно независимые алгебраические числа. Следовательно, с помощью процедуры п. 6.4 мы можем доказать, что специальный слой $J_{v}^{\mathrm{NS}}$ является обыкновенным.

6.7. Предположим, что $K$-вполне вещественное поле, и $d / e=2 m$-четное число, $m \geqslant 2$. По теореме Ю.Г. Зархина (п. 5.4) $\mathrm{Lie} \mathrm{Hg}\left(J^{\mathrm{NS}} \otimes_{k} \mathbb{C}\right) \otimes \overline{\mathbb{Q}}$ - полупростая алгебра Ли типа $D_{m} \times \cdots \times D_{m}=D_{m}^{e}(m \geqslant 2)$. 
Предположим, например, что $e=2$. Тогда $\operatorname{Lie} \operatorname{Hg}\left(J^{\mathrm{NS}} \otimes_{k} \mathbb{C}\right) \otimes_{\mathbb{Q}} \overline{\mathbb{Q}}$ имеет тип $D_{m} \times D_{m}$ и

$$
\begin{aligned}
& H_{\mathrm{et}}^{1}\left(J^{\mathrm{NS}} \otimes \bar{k}, \mathbb{Q}_{l}\right) \otimes \overline{\mathbb{Q}_{l}}= 2^{2 m-1}\left(E\left(\omega_{m-1}^{(1)}+\omega_{m-1}^{(2)}\right) \oplus E\left(\omega_{m}^{(1)}+\omega_{m}^{(2)}\right)\right. \\
&\left.\oplus E\left(\omega_{m-1}^{(1)}+\omega_{m}^{(2)}\right) \oplus E\left(\omega_{m}^{(1)}+\omega_{m-1}^{(2)}\right)\right)
\end{aligned}
$$

$[23$, формулы (4.2.4), (4.2.5)]. Поэтому можно считать, что

$$
\begin{aligned}
\Delta=\{ & \left.\lambda \alpha_{1}^{a_{1}} \ldots \alpha_{m}^{a_{m}} \mid a_{j} \in\{ \pm 1\}, \operatorname{Card}\left(\left\{j \mid a_{j}=-1\right\}\right)=1(\bmod 2)\right\} \\
& \times\left\{\alpha_{m+1}^{a_{m+1}} \ldots \alpha_{2 m}^{a_{2 m}} \mid a_{j} \in\{ \pm 1\}, \operatorname{Card}\left(\left\{j \mid a_{j}=-1\right\}\right)=1(\bmod 2)\right\} \\
& \cup\left\{\lambda \alpha_{1}^{a_{1}} \ldots \alpha_{m}^{a_{m}} \mid a_{j} \in\{ \pm 1\}, \operatorname{Card}\left(\left\{j \mid a_{j}=-1\right\}\right)=0(\bmod 2)\right\} \\
& \times\left\{\alpha_{m+1}^{a_{m+1}} \ldots \alpha_{2 m}^{a_{2 m}} \mid a_{j} \in\{ \pm 1\}, \operatorname{Card}\left(\left\{j \mid a_{j}=-1\right\}\right)=0(\bmod 2)\right\} \\
& \cup\left\{\lambda \alpha_{1}^{a_{1}} \ldots \alpha_{m}^{a_{m}} \mid a_{j} \in\{ \pm 1\}, \operatorname{Card}\left(\left\{j \mid a_{j}=-1\right\}\right)=1(\bmod 2)\right\} \\
& \times\left\{\alpha_{m+1}^{a_{m+1}} \ldots \alpha_{2 m}^{a_{2 m}} \mid a_{j} \in\{ \pm 1\}, \operatorname{Card}\left(\left\{j \mid a_{j}=-1\right\}\right)=0(\bmod 2)\right\} \\
& \cup\left\{\lambda \alpha_{1}^{a_{1}} \ldots \alpha_{m}^{a_{m}} \mid a_{j} \in\{ \pm 1\}, \operatorname{Card}\left(\left\{j \mid a_{j}=-1\right\}\right)=0(\bmod 2)\right\} \\
& \times\left\{\alpha_{m+1}^{a_{m+1}} \ldots \alpha_{2 m}^{a_{2 m}} \mid a_{j} \in\{ \pm 1\}, \operatorname{Card}\left(\left\{j \mid a_{j}=-1\right\}\right)=1(\bmod 2)\right\} \\
= & \left\{\lambda \alpha_{1}^{ \pm 1} \ldots \alpha_{2 m}^{ \pm 1}\right\},
\end{aligned}
$$

где $\lambda, \alpha_{1}, \ldots, \alpha_{2 m} \in \overline{\mathbb{Q}}^{\times}$мультипликативно независимы. Следовательно, с помощью процедуры п. 6.4 мы можем доказать, что специальный слой $J_{v}^{\mathrm{NS}}$ является обыкновенным.

Если $е$ произвольно, то мы имеем разложение

$$
\begin{aligned}
H_{\mathrm{et}}^{1}\left(J^{\mathrm{NS}} \otimes \bar{k}, \mathbb{Q}_{l}\right) \otimes \overline{\mathbb{Q}_{l}}= & \bigoplus_{\substack{i_{1}, \ldots, i_{e} \in\{m-1, m\} \\
\operatorname{Card}\left(\left\{i_{p} \mid i_{p}=m\right\}\right)=1(\bmod 2)}} E\left(\omega_{i_{1}}^{(1)}+\cdots+\omega_{i_{e}}^{(e)}\right) \\
& \times \bigoplus_{\substack{i_{1}, \ldots, i_{e} \in\{m-1, m\} \\
\operatorname{Card}\left(\left\{i_{p} \mid i_{p}=m\right\}\right)=0(\bmod 2)}} E\left(\omega_{i_{1}}^{(1)}+\cdots+\omega_{i_{e}}^{(e)}\right)
\end{aligned}
$$

$\left[24\right.$, п. 1.7]. Легко понять, что в этом случае $\Delta=\left\{\lambda \alpha_{1}^{ \pm 1} \ldots \alpha_{m e}^{ \pm 1}\right\}$, где $\lambda, \alpha_{1}, \ldots, \alpha_{m e} \in \overline{\mathbb{Q}}^{\times}$мультипликативно независимы. Следовательно, с помощью процедуры п. 6.4 мы можем доказать, что специальньй слой $J_{v}^{\mathrm{NS}}$ является обыкновенным. Теорема 0.10 доказана.

\section{§ 7. Некоторые арифметические свойства собственных чисел $\Phi$ робениуса}

7.1. Мы начинаем доказательство теоремы 12 . Пусть $g_{l}=\operatorname{Lie} \operatorname{Im}\left(\rho_{l}\right)$. Мы будем обозначать через $g_{l}^{s s}$ полупростую часть $g_{l}$.

7.2. Лемма. Пусть $J$-абсолютно простое $d$-мерное абелево многообразие над достаточно большим числовым полем $k$. Предположим, что выполняется одно из следующих условий: 
1) $d$ - простое число, $d \geqslant 3$;

2) $J$-абелево многообразие CM-типа, и размерность над $\mathbb{Q}$ группы Ходжа $\mathrm{Hg}\left(J \otimes_{k} \mathbb{C}\right)$ равна $d=\operatorname{dim}_{k} J$.

Тогда для любой неархимедовой точки $v$ поля $k$ из некоторого множества $S_{\text {non-Arch }}^{\text {genic }}(J, k, l)$ точек с плотностью Дирихле 1 мы можем считать, что $\Delta=\left\{\lambda \alpha_{i}^{ \pm 1} \mid i=1, \ldots, d\right\}$, где $\lambda, \alpha_{1}, \ldots, \alpha_{d}$ - мультипликативно независимые алгебраические числа, $\lambda^{2}=p_{v}$.

ДокАЗАТЕЛЬСТво. Фиксируем $k$-поляризацию на $J$. Обозначим через

$$
\Psi: H_{\mathrm{et}}^{1}\left(J \otimes \bar{k}, \mathbb{Q}_{l}\right) \times H_{\mathrm{et}}^{1}\left(J \otimes \bar{k}, \mathbb{Q}_{l}\right) \rightarrow \mathbb{Q}_{l}(-1)
$$

индуцированную невырожденную кососимметрическую форму на $H_{\mathrm{et}}^{1}\left(J \otimes \bar{k}, \mathbb{Q}_{l}\right)$. Естественное разложение $G_{V_{l}}=\mathrm{G}_{m} \cdot\left(G_{V_{l}} \cap \mathrm{Sp}(\Psi)\right)$ и условия п. 2.1 показывают, что $\Delta=\left\{\lambda \alpha_{i}^{ \pm 1} \mid i=1, \ldots, d\right\}$, где $\lambda, \alpha_{1}, \ldots, \alpha_{d}$ - алгебраические числа [26, пा. 2.7 , 2.8]. Мы должны доказать, что эти числа мультипликативно независимы. В силу $2.1,6)$ достаточно проверить, что $\operatorname{rank}\left(G_{V_{l}}\right)=d+1$. Напомним, что $\operatorname{rank}\left(G_{V_{l}}\right)=$ $\operatorname{rank}\left(g_{l}\right)$.

Согласно классификации Альберта $[13, \S 21]$ мы имеем следующие варианты: $\operatorname{End}^{0}(J \otimes \bar{k})=\mathbb{Q}, \quad \operatorname{End}^{0}(J \otimes \bar{k})$ - вполне вешественное поле степени $d$ над $\mathbb{Q}$, $\operatorname{End}^{0}(J \otimes \bar{k})$ - мнимое квадратичное расширение $\mathbb{Q}, \operatorname{End}^{0}(J \otimes \bar{k})$ - чисто мнимое квадратичное расширение вполне вешественного поля степени $d$ над $\mathbb{Q}(\mathrm{CM}$-поле степени $2 d$ в терминологии $[20$, гл. 5$])$.

Предположим, что $\operatorname{End}^{0}(J \otimes \bar{k})=\mathbb{Q}$. В этом случае $g_{l}^{s s} \otimes \overline{\mathbb{Q}_{l}}$ - простая алгебра Ли типа $C_{d}$ (см. $\left[7\right.$, п. 6], [22]), $\operatorname{dim}_{\mathbb{Q}_{l}} \operatorname{Cent}\left(g_{l}\right)=1$. Поэтому $\operatorname{rank}\left(g_{l}\right)=d+1$.

Предположим, что $\operatorname{End}^{0}(J \otimes \bar{k})$ - вполне вешественное поле степени $d$ над $\mathbb{Q}$. В этом случае $g_{l}^{s s} \otimes \overline{\mathbb{Q}_{l}}-$ полупростая алгебра Ли типа $A_{1} \times \cdots \times A_{1}=A_{1}^{d}[22]$, $\operatorname{dim}_{\mathbb{Q}_{l}} \operatorname{Cent}\left(g_{l}\right)=1$. Поэтому $\operatorname{rank}\left(g_{l}\right)=d+1$.

Предположим, что $\operatorname{End}^{0}(J \otimes \bar{k})$ - мнимое квадратичное расширение $\mathbb{Q}$. В этом случае $g_{l}^{s s} \otimes \overline{\mathbb{Q}}_{l}-$ простая алгебра Ли типа $A_{d-1}$ (см. [22], [6]), $\operatorname{dim}_{\mathbb{Q}_{l}} \operatorname{Cent}\left(g_{l}\right)=$ $2[6]$. Поэтому $\operatorname{rank}\left(g_{l}\right)=d+1$.

Предположим, что $\operatorname{End}^{0}(J \otimes \bar{k})$ - чисто мнимое квадратичное расширение вполне вешественного поля степени $d$ над $\mathbb{Q}$. В этом случае $\operatorname{rank}\left(g_{l}\right)=d+1$ [21].

Мультипликативная независимость $\lambda, \alpha_{1}, \ldots, \alpha_{d}$ влечет соотношение

$$
\eta=\lambda^{2} \Leftrightarrow \operatorname{Card}\left(T_{\eta}(\Delta) \cap \Delta\right)=\operatorname{Card}(\Delta) .
$$

Следовательно, $\lambda^{2} \in \mathbb{Q}$ имеет абсолютную величину $p_{v}$ (по условию $2.1,3$ ) и в силу известной теоремы Вейля $[13, \S 21$, теорема 4$]$ ), поэтому мы имеем равенство $\lambda^{2}= \pm p_{v}$.

Пусть $\rho$-комплексное сопряжение на $\overline{\mathbb{Q}}$, индуцированное некоторым вложением $\overline{\mathbb{Q}} \rightarrow \mathbb{C}$. Мы утверждаем, что

$$
\rho\left(\lambda \alpha_{i}\right)=\lambda \alpha_{i}^{-1} \text {. }
$$

Действительно, по теореме Вейля $\lambda \alpha_{1} \cdot \rho\left(\lambda \alpha_{1}\right)=p_{v}$ в силу соотношения $\left.2.1,3\right)$. Предположим, что $\rho\left(\lambda \alpha_{1}\right)=\lambda \alpha_{1}$. Тогда $\lambda^{2} \alpha_{1}^{2}=p_{v}, \lambda^{4} \alpha_{1}^{4}=p_{v}^{2}, p_{v}^{2} \alpha_{1}^{4}=p_{v}^{2}$, 
$\alpha_{1}^{4}=1$, что противоречит мультипликативной независимости $\lambda, \alpha_{1}, \ldots, \alpha_{d}$. Предположим, что $\rho\left(\lambda \alpha_{1}\right)=\lambda \alpha_{2}$. Тогда мы имеем $\lambda^{2} \alpha_{1} \alpha_{2}=p_{v}, \lambda^{4} \alpha_{1}^{2} \alpha_{2}^{2}=p_{v}^{2}$, $p_{v}^{2} \alpha_{1}^{2} \alpha_{2}^{2}=p_{v}^{2}, \alpha_{1}^{2} \alpha_{2}^{2}=1$ вопреки мультипликативной независимости $\lambda, \alpha_{1}, \ldots, \alpha_{d}$.

Поэтому $\rho\left(\lambda \alpha_{1}\right)=\lambda \alpha_{1}^{-1}$, и утверждение (7.2.1) доказано. С другой стороны, по теореме Вейля $p_{v}=\lambda \alpha_{1} \cdot \rho\left(\lambda \alpha_{1}\right)=\lambda \alpha_{1} \lambda \alpha_{1}^{-1}=\lambda^{2}$. Лемма доказана.

7.3. Предположим, что неархимедова точка $v$ удовлетворяет условиям п. 2.1. Согласно теории Хонды-Тэйта [32] $p_{v}$-число Вейля $\lambda \alpha_{1}$ определяет класс изогений простого абелева многообразия $A$ над конечньм полем $\mathbb{F}_{p_{v}}$. Поскольку $\mathbb{F}_{p_{v}}-$ простое поле, мы имеем соотношения

$$
\operatorname{End}^{0}(A)=F=\mathbb{Q}\left(\lambda \alpha_{1}\right) ;
$$

для любой точки $w$ поля $F$, лежашей над $p_{v}$,

$$
\frac{w\left(\lambda \alpha_{1}\right)}{w\left(p_{v}\right)} \cdot\left[F_{w}: \mathbb{Q}_{p_{v}}\right] \in \mathbb{Z},
$$

где $F_{w}$ обозначает пополнение $F$ относительно $w$; более того, в силу леммы 7.2 $F$ является мнимым квадратичным расширением вполне вещественного поля $F_{0}=$ $\mathbb{Q}\left(\lambda \alpha_{1}+\lambda \alpha_{1}^{-1}\right)[32$, замечание 2].

Обозначим через $F^{\prime}$ минимальное нормальное расширение поля $\mathbb{Q}$, содержащее $F$.

7.4. Лемма. Имеем следующие соотношения:

для любой точки $w$ поля $F$, лежащей над $p_{v}$,

$$
\frac{w\left(\lambda \alpha_{1}\right)}{w\left(p_{v}\right)}+\frac{\rho w\left(\lambda \alpha_{1}\right)}{\rho w\left(p_{v}\right)}, \quad\left\{(\rho w=w) \Rightarrow \frac{w\left(\lambda \alpha_{1}\right)}{w\left(p_{v}\right)}=\frac{1}{2}\right\}
$$

для любой точки $w^{\prime}$ поля $F^{\prime}$, лежащей над $w$,

$$
\frac{w^{\prime}\left(\lambda \alpha_{1}\right)}{w^{\prime}\left(p_{v}\right)}=\frac{w\left(\lambda \alpha_{1}\right)}{w\left(p_{v}\right)}
$$

для любой точки $w^{\prime}$ поля $F^{\prime}$, лежащей над $p_{v}$, и для любого әлемента $\sigma \in \operatorname{Gal}\left(F^{\prime} / \mathbb{Q}\right)$

$$
\frac{w^{\prime}\left(\lambda \alpha_{1}\right)}{w^{\prime}\left(p_{v}\right)}=\frac{\sigma w^{\prime}\left(\sigma\left(\lambda \alpha_{1}\right)\right)}{\sigma w^{\prime}\left(p_{v}\right)}
$$

для әлементов $\delta_{1}, \delta_{2} \in \Delta$

$$
\left\{\left(\forall w^{\prime} \mid p_{v}\right) \frac{w^{\prime}\left(\delta_{1}\right)}{w^{\prime}\left(p_{v}\right)}=\frac{w^{\prime}\left(\delta_{2}\right)}{w^{\prime}\left(p_{v}\right)}\right\} \Rightarrow \delta_{1} / \delta_{2}
$$

- корень из единиць;

$$
\rho \in \operatorname{Cent}\left(\operatorname{Gal}\left(F^{\prime} / \mathbb{Q}\right)\right) .
$$

Соотношения (7.4.1)-(7.4.4) следуют из равенства Вейля $\lambda \alpha_{1} \cdot \rho\left(\lambda \alpha_{1}\right)=p_{v}$ и из элементарных свойств нормирований [5], [33]. Соотношение (7.4.5) является хорошо известным свойством СМ-полей $[20$, гл. $5, \S 5.5$, предложения $5.11,5.12]$.

В дальнейшем мы отождествляем точку $w \mid p_{v}$ с нормированием, нормализованным равенством $w\left(p_{v}\right)=1$, и отождествляем $w^{\prime} \mid p_{v}$ с нормированием, нормализованным соотношением $w^{\prime}\left(p_{v}\right)=1$. Очевидно, что эти отождествления совместимы с действием групп Галуа на множествах нормирований и неархимедовых точек. 
7.5. ЛЕмма. Пусть $J$-абсолютно простое абелево многообразие CM-типа над достаточно большим числовым полем $k$. Если $\operatorname{dim}_{k} J$ - простое число или если размерность над $\mathbb{Q}$ группы Ходжса $\operatorname{Hg}\left(J \otimes_{k} \mathbb{C}\right)$ равна $\operatorname{dim}_{k} J$, то для любой неархимедовой точки $v$ поля $k$ из некоторого множества $S_{\text {non-Arch }}^{\text {generic }}(J, k, l)$ точек с плотностью Дирихле 1 специальный слой $J_{v}$ минимальной модели Нерона $J$ является обыкновенным простым абелевым многообразием.

ДокаЗАТЕЛЬСТво. Пусть $K=\operatorname{End}^{0}(J \otimes \bar{k})$. Можно считать, что $K=$ $\operatorname{End}^{0}(J), K \subset k, p_{v}$ неразветвлено в $k, v$ удовлетворяет условиям п. 2.1. Из соотношения $\operatorname{Norm}_{k / \mathbb{Q}}(v)=p_{v}$ следует, что $p_{v}$ полностью распадается в $k$. Поэтому $p_{v}$ полностью распадается в $K: K \otimes_{\mathbb{Q}} \mathbb{Q}_{p_{v}} \simeq \mathbb{Q}_{p_{v}} \times \cdots \times \mathbb{Q}_{p_{v}}$. Заметим, что отображение редукции $K=\operatorname{End}^{0}(J) \rightarrow \operatorname{End}^{0}\left(J_{v}\right)$ является вложением $K$ в произведение полей. Это следует из факта, что $\operatorname{Pc}_{J}\left(\rho_{l}\left(F_{\bar{v}}^{-1}\right), t\right)$ не имеет кратных корней в силу леммы 7.2 [33, лемма 1.0.3]. В частности, мы имеем вложение $K \subset F=\mathbb{Q}\left(\lambda \alpha_{1}\right)$. Поэтому $F=K, J_{v}$-простое абелево многообразие, и $p_{v}$ полностью распадается в $F: F \otimes_{\mathbb{Q}} \mathbb{Q}_{p_{v}} \simeq \mathbb{Q}_{p_{v}} \times \cdots \times \mathbb{Q}_{p_{v}}$. С другой стороны, $F \otimes_{\mathbb{Q}} \mathbb{Q}_{p_{v}}=\bigoplus_{w \mid p_{v}} F_{w}[5$, гл. 2 , $\S 10$, теорема 10.2]. Поэтому $\left[F_{w}: \mathbb{Q}_{p_{v}}\right]=1$ для всех точек $w$ поля $F$, лежаших над $p_{v}$. В силу (7.3.1) $w\left(\lambda \alpha_{1}\right) \in\{0,1\}$ для всех точек $w \mid p_{v}$. Из леммы 7.4 мы получаем соотношение $w^{\prime}\left(\lambda \alpha_{i}^{ \pm 1}\right) \in\{0,1\}(i=1, \ldots, d)$. Поэтому $J_{v}$ - обыкновенное абелево многообразие.

7.6. В дальнейшем мы считаем, что $J$ - абсолютно простое трехмерное абелево многообразие, удовлетворяюшее условиям теоремы $0.12, K=\operatorname{End}^{0}(J \otimes \bar{k})-$ мнимое квадратичное расширение поля $\mathbb{Q}$.

7.7. Лемма. Имеем

$$
\begin{gathered}
\operatorname{Pc}_{J}\left(\rho_{l}\left(F_{\bar{v}}^{-1}\right), t\right)=\operatorname{det}\left(t-\rho_{l}\left(F_{\bar{v}}^{-1}\right)\right)=t^{6}-\operatorname{Tr}\left(\rho_{l}\left(F_{\bar{v}}^{-1}\right)\right) \cdot t^{5}+\operatorname{Tr}\left(\rho_{l}^{\wedge}\left(F_{\bar{v}}^{-1}\right)\right) \cdot t^{4} \\
-\operatorname{Tr}\left(\rho_{l}^{\wedge 3}\left(F_{\bar{v}}^{-1}\right)\right) \cdot t^{3}+\operatorname{Tr}\left(\rho_{l}^{\wedge 4}\left(F_{\bar{v}}^{-1}\right)\right) \cdot t^{2}-\operatorname{Tr}\left(\rho_{l}^{\wedge 5}\left(F_{\bar{v}}^{-1}\right)\right) \cdot t+\operatorname{det}\left(\rho_{l}\left(F_{\bar{v}}^{-1}\right)\right),
\end{gathered}
$$

zдe

$$
\begin{aligned}
\operatorname{Tr}\left(\rho_{l}\left(F_{\bar{v}}^{-1}\right)\right)= & \lambda \alpha_{1}+\lambda \alpha_{1}^{-1}+\lambda \alpha_{2}+\lambda \alpha_{2}^{-1}+\lambda \alpha_{3}+\lambda \alpha_{3}^{-1}, \\
\operatorname{Tr}\left(\rho_{l}^{\wedge}\left(F_{\bar{v}}^{-1}\right)\right)= & 3 \lambda^{2}+\left(\lambda \alpha_{1}+\lambda \alpha_{1}^{-1}\right)\left(\lambda \alpha_{2}+\lambda \alpha_{2}^{-1}\right) \\
& \quad+\left(\lambda \alpha_{1}+\lambda \alpha_{1}^{-1}\right)\left(\lambda \alpha_{3}+\lambda \alpha_{3}^{-1}\right)+\left(\lambda \alpha_{2}+\lambda \alpha_{2}^{-1}\right)\left(\lambda \alpha_{3}+\lambda \alpha_{3}^{-1}\right), \\
\operatorname{Tr}\left(\rho_{l}^{\wedge 3}\left(F_{\bar{v}}^{-1}\right)\right)= & 2 \lambda^{2} \cdot \operatorname{Tr}\left(\rho_{l}\left(F_{\bar{v}}^{-1}\right)\right)+\left(\lambda \alpha_{1}+\lambda \alpha_{1}^{-1}\right)\left(\lambda \alpha_{2}+\lambda \alpha_{2}^{-1}\right)\left(\lambda \alpha_{3}+\lambda \alpha_{3}^{-1}\right) .
\end{aligned}
$$

Это следствие элементарных вычислений.

7.8. В силу условия (2.1.4) и леммы 7.2 для неархимедовой точки $v \in S_{\text {non-Arch }}^{\text {generic }}$ мы имеем

$$
\begin{aligned}
& \lambda \alpha_{1}+\lambda \alpha_{1}^{-1}+\lambda \alpha_{2}+\lambda \alpha_{2}^{-1}+\lambda \alpha_{3}+\lambda \alpha_{3}^{-1} \neq 0\left(\bmod p_{v}\right) \\
& \left(\lambda \alpha_{1}+\lambda \alpha_{1}^{-1}\right)\left(\lambda \alpha_{2}+\lambda \alpha_{2}^{-1}\right)+\left(\lambda \alpha_{1}+\lambda \alpha_{1}^{-1}\right)\left(\lambda \alpha_{3}+\lambda \alpha_{3}^{-1}\right) \\
& \quad+\left(\lambda \alpha_{2}+\lambda \alpha_{2}^{-1}\right)\left(\lambda \alpha_{3}+\lambda \alpha_{3}^{-1}\right) \neq 0 \quad\left(\bmod p_{v}\right)
\end{aligned}
$$




\section{§ 8. Случай неприводимого характеристического полинома}

8.1. Мы предполагаем, что характеристический полином $P(t)=$ $\operatorname{Pc}_{J}\left(\rho_{l}\left(F_{\bar{v}}^{-1}\right), t\right)$ неприводим над полем $\mathbb{Q}$. Из неприводимости $P(t)$ и леммы 7.2 следует, что $[F: \mathbb{Q}]=6$ и отображение редукции $K=\operatorname{End}^{0}(J) \rightarrow \operatorname{End}^{0}\left(J_{v}\right)=F$ является вложением. В дальнейшем мы обозначаем через $x_{0}, y_{0}, \ldots$ точки $F_{0}$, лежащие над $p_{v}$; через $x, \rho x$ - точки $F$, лежащие над $x_{0}$; через $x^{\prime}, x^{\prime \prime} \ldots-$ точки $F^{\prime}$, лежащие над $x$. Напомним, что $F \otimes_{\mathbb{Q}} \mathbb{Q}_{p_{v}}=\bigoplus_{w \mid p_{v}} F_{w}, F_{0} \otimes_{\mathbb{Q}} \mathbb{Q}_{p_{v}}=$ $\bigoplus_{w_{0} \mid p_{v}} F_{0 w_{0}}[5$, гл. $2, \S 10$, теорема 10.2].

Пусть $F_{0}^{\prime}$ - минимальное нормальное расширение поля $\mathbb{Q}$, содержашее вполне вещественное кубическое поле $F_{0}=\mathbb{Q}\left(\lambda \alpha_{1}+\lambda \alpha_{1}^{-1}\right)$. Очевидно, что $\operatorname{Gal}\left(F^{\prime} / \mathbb{Q}\right)$ действует транзитивно на $\left\{\lambda \alpha_{1}+\lambda \alpha_{1}^{-1}, \lambda \alpha_{2}+\lambda \alpha_{2}^{-1}, \lambda \alpha_{3}+\lambda \alpha_{3}^{-1}\right\}$, где $\lambda \alpha_{1}+\lambda \alpha_{1}^{-1}$, $\lambda \alpha_{2}+\lambda \alpha_{2}^{-1}, \lambda \alpha_{3}+\lambda \alpha_{3}^{-1}$ порождают $F_{0}^{\prime}$ над $\mathbb{Q}$. Поэтому можно считать, что $\operatorname{Gal}\left(F^{\prime} / \mathbb{Q}\right)$ содержит элемент $\sigma$ порядка 3. Легко видеть, что $F=K\left(F_{0}\right)$. Мы имеем $K \cap F_{0}^{\prime}=\mathbb{Q}$, потому что $F_{0}^{\prime}$ - вполне вещественное поле. Следовательно, $K$ и $F_{0}^{\prime}$ линейно разделены над $\mathbb{Q}, K\left(F_{0}^{\prime}\right)$ - нормальное расширение $\mathbb{Q}$,

$$
\operatorname{Gal}\left(K\left(F_{0}^{\prime}\right) / \mathbb{Q}\right)=\operatorname{Gal}(K / \mathbb{Q}) \times \operatorname{Gal}\left(F_{0}^{\prime} / \mathbb{Q}\right)
$$

$\left[3\right.$, гл. $5, \S 10$, п. 4 , следствие 2]. Очевидно, что $F^{\prime}=K\left(F_{0}^{\prime}\right)$. Поэтому (8.1.1) дает соотношение

$$
\operatorname{Gal}\left(F^{\prime} / \mathbb{Q}\right)=\operatorname{Gal}(K / \mathbb{Q}) \times \operatorname{Gal}\left(F_{0}^{\prime} / \mathbb{Q}\right)=\{1, \rho\} \times \operatorname{Gal}\left(F_{0}^{\prime} / \mathbb{Q}\right)
$$

Более того, мы можем считать, что

$$
\sigma\left(\lambda \alpha_{1}^{n}\right)=\lambda \alpha_{2}^{n}, \quad \sigma\left(\lambda \alpha_{2}^{n}\right)=\lambda \alpha_{3}^{n}, \quad \sigma\left(\lambda \alpha_{3}^{n}\right)=\lambda \alpha_{1}^{n} \quad(n= \pm 1) .
$$

8.2. Предположим, что $x_{0}$ - единственная точка кубического поля $F_{0}$, лежащая над $p_{v}$. Имеем два варианта: $x=\rho x$ - единственная точка $F$, лежащая над $p_{v}$, или $x, \rho x$ - различные точки $F$, лежашие над $p_{v}$. Изображаем эти варианты следуюшими диаграммами:

$$
\begin{gathered}
x=\rho x \rightarrow x_{0} \rightarrow\left(p_{v}\right) ; \\
x \\
\downarrow \\
\rho x \rightarrow x_{0} \rightarrow\left(p_{v}\right) .
\end{gathered}
$$

8.3. Рассмотрим случай (8.2.1). В силу леммы 7.4 мы имеем $x\left(\lambda \alpha_{1}^{ \pm 1}\right)=1 / 2$, поэтому $\alpha_{1}^{2}-$ корень из единицы, что противоречит мультипликативной независимости $\lambda, \alpha_{1}, \alpha_{2}, \alpha_{3}$.

8.4. Рассмотрим случай (8.2.2). Мы имеем $\left[F_{x}: \mathbb{Q}_{p_{v}}\right]=\left[F_{\rho x}: \mathbb{Q}_{p_{v}}\right]=3$. Из (7.3.1) следует, что $x\left(\lambda \alpha_{1}\right) \in\{0,1 / 3,2 / 3,1\}, \rho x\left(\lambda \alpha_{1}\right) \in\{0,1 / 3,2 / 3,1\}$. Предположим, что $x\left(\lambda \alpha_{1}\right) \in\{1 / 3,2 / 3\}$. Тогда в силу соотношения (7.4.1) имеем $\rho x\left(\lambda \alpha_{1}\right) \in\{1 / 3,2 / 3\}$. В силу (7.4.2) для любой точки $x^{\prime}$ поля $F^{\prime}$, лежащей над $p_{v}$, 
имеем $x^{\prime}\left(\lambda \alpha_{1}\right) \in\{1 / 3,2 / 3\}$. Заметим, что $\lambda \alpha_{1}^{ \pm 1}, \lambda \alpha_{2}^{ \pm 1}, \lambda \alpha_{a}^{ \pm 1}$ сопряжены друг другу над $\mathbb{Q}$, поэтому в силу (7.4.3) для любой точки $x^{\prime}$ поля $F^{\prime}$ имеем $x^{\prime}\left(\lambda \alpha_{1,2,3}^{ \pm 1}\right) \in$ $\{1 / 3,2 / 3\}$. Мы видим, что $\left(\forall x^{\prime} \mid p_{v}\right) x^{\prime}\left(\operatorname{Tr}\left(\rho_{l}\left(F_{\bar{v}}^{-1}\right)\right)\right)=x^{\prime}\left(\sum \lambda \alpha_{1,2,3}^{ \pm 1}\right)>0$. Поэтому $p_{v}$ делит $\operatorname{Tr}\left(\rho_{l}\left(F_{\bar{v}}^{-1}\right)\right)$, что противоречит условию $\left.2.1,4\right)$.

Видим, что $x\left(\lambda \alpha_{1}\right) \in\{0,1\}$. В силу леммы 7.4 для любой точки $x^{\prime}$ поля $F^{\prime}$ имеем $x^{\prime}\left(\lambda \alpha_{1,2,3}^{ \pm 1}\right) \in\{0,1\}$. Поэтому $J_{v}$ - обыкновенное абелево многообразие.

8.5. Предположим, что $x_{0}, y_{0}$ - все точки кубического поля $F_{0}$, лежашие над $p_{v}$. Заметим, что $F_{0}^{\prime} \neq F_{0}$ (иначе получили бы, что все точки $w_{0} \mid p_{v}$ сопряжены друг другу, и число таких точек равно 1 или 3 ). Имеются следующие варианты:

$$
\begin{aligned}
& x=\rho x \rightarrow x_{0} \rightarrow\left(p_{v}\right) \leftarrow y_{0} \leftarrow y=\rho y ; \\
& \begin{aligned}
x & \\
\downarrow & \\
\rho x \rightarrow x_{0} & \rightarrow\left(p_{v}\right) \leftarrow y_{0} \leftarrow y=\rho y ;
\end{aligned} \\
& \begin{array}{c}
y \\
\downarrow \\
\downarrow=\rho x \rightarrow x_{0} \rightarrow\left(p_{v}\right) \leftarrow y_{0} \leftarrow \rho y ;
\end{array} \\
& x \quad y \\
& \downarrow \downarrow \downarrow \\
& \rho x \rightarrow x_{0} \rightarrow\left(p_{v}\right) \leftarrow y_{0} \leftarrow \rho y .
\end{aligned}
$$

8.6. Очевидно, что $F_{0} \otimes_{\mathbb{Q}} \mathbb{Q}_{p_{v}}=F_{0 x_{0}} \oplus F_{0 y_{0}}$. С другой стороны, $\operatorname{Gal}\left(F_{0}^{\prime} / F_{0}\right)=$ $\{1, \tau\}$, где $\tau$ - элемент порядка 2. Мы имеем $\lambda \alpha_{1}+\lambda \alpha_{1}^{-1} \in F_{0}$, поэтому

$$
\begin{aligned}
& \tau\left(\lambda \alpha_{1}+\lambda \alpha_{1}^{-1}\right)=\lambda \alpha_{1}+\lambda \alpha_{1}^{-1}, \\
& \tau\left(\lambda \alpha_{2}+\lambda \alpha_{2}^{-1}\right)=\lambda \alpha_{3}+\lambda \alpha_{3}^{-1}, \\
& \tau\left(\lambda \alpha_{3}+\lambda \alpha_{3}^{-1}\right)=\lambda \alpha_{2}+\lambda \alpha_{2}^{-1} .
\end{aligned}
$$

Мы хотим пополнить диаграмму $x_{0} \rightarrow\left(p_{v}\right) \leftarrow y_{0}$ точками поля $F_{0}^{\prime}$. Можно рассматривать только три варианта такого пополнения:

$$
\begin{gathered}
\tau x_{0}^{\prime}=x_{0}^{\prime} \rightarrow x_{0} \rightarrow\left(p_{v}\right) \leftarrow y_{0} \leftarrow y_{0}^{\prime}=\tau y_{0}^{\prime} ; \\
x_{0}^{\prime} \\
\downarrow \\
y_{0}^{\prime} \\
\downarrow \\
\begin{array}{c}
\downarrow \\
\end{array} \\
x_{0}^{\prime} \rightarrow\left(p_{v}\right) \leftarrow y_{0} \leftarrow \tau y_{0}^{\prime} ; \\
y_{0}^{\prime} \\
\downarrow \\
\tau x_{0}^{\prime}=x_{0}^{\prime} \rightarrow x_{0} \rightarrow\left(p_{v}\right) \leftarrow y_{0} \leftarrow \tau y_{0}^{\prime} .
\end{gathered}
$$

$\mathrm{C}$ другой стороны, $F_{0}^{\prime} / \mathbb{Q}$ - нормальное расширение с группой Галуа $\mathrm{S}_{3}$, поэтому все точки $F_{0}^{\prime}$, лежашие над $p_{v}$, сопряжены друг другу, и число таких точек равно $2,3,6$. Мы видим, что могут появиться только диаграммы (8.6.1) и (8.6.2). 
$\mathrm{B}$ случае (8.6.1) мы имеем $\tau \in \operatorname{Stab}\left(x_{0}^{\prime}\right) \cap \operatorname{Stab}\left(y_{0}^{\prime}\right)$. Поэтому $\left\{1, \sigma, \sigma^{2}\right\}$ действует транзитивно на $\left\{x_{0}^{\prime}, y_{0}^{\prime}\right\}$. Это невозможно. Следовательно, мы имеем только один вариант (8.6.2).

8.7. Рассмотрим случай (8.5.1). В силу леммы 7.4 имеем $x\left(\lambda \alpha_{1}^{ \pm 1}\right)=1 / 2=$ $y\left(\lambda \alpha_{1}^{ \pm 1}\right)$, поэтому $\alpha_{1}^{2}-$ корень из единицы, что противоречит мультипликативной независимости $\lambda, \alpha_{1}, \alpha_{2}, \alpha_{3}$.

8.8. Рассмотрим случай (8.5.2) и часть пополнения диаграммы (8.6.2) точками поля $F^{\prime}$ :

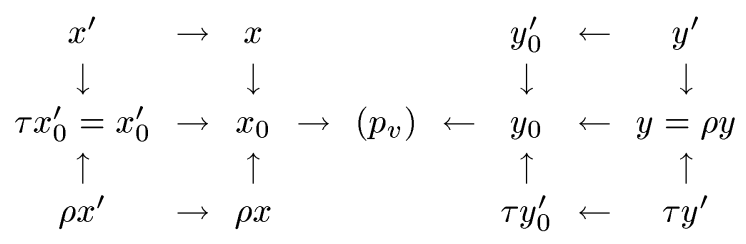

Имеем $2=\left[F_{0 x_{0}^{\prime}}^{\prime}: \mathbb{Q}_{p_{v}}\right]=\left[F_{0 y_{0}^{\prime}}^{\prime}: \mathbb{Q}_{p_{v}}\right]=\left[F_{0 \tau y_{0}^{\prime}}^{\prime}: \mathbb{Q}_{p_{v}}\right], F_{0 x_{0}^{\prime}}^{\prime}=F_{0}^{\prime} \otimes_{F_{0}} F_{0 x_{0}}$, поэтому

$$
\left[F_{0 x_{0}}: \mathbb{Q}_{p_{v}}\right]=1, \quad\left[F_{0 y_{0}}: \mathbb{Q}_{p_{v}}\right]=2 .
$$

С другой стороны,

$$
F \otimes_{\mathbb{Q}} \mathbb{Q}_{p_{v}}=F_{x} \oplus F_{\rho x} \oplus F_{y}, \quad F_{x} \oplus F_{\rho x}=F \otimes_{F_{0}} F_{0 x_{0}}, \quad F_{y}=F \otimes_{F_{0}} F_{0 y_{0}},
$$

поэтому (8.8.2) влечет $\left[F_{x}: \mathbb{Q}_{p_{v}}\right]=\left[F_{\rho x}: \mathbb{Q}_{p_{v}}\right]=1,\left[F_{y}: \mathbb{Q}_{p_{v}}\right]=4$. В силу (7.3.1) и (7.4.1) имеем $x\left(\lambda \alpha_{1}\right) \in\{0,1\}, \rho x\left(\lambda \alpha_{1}\right)=1-x\left(\lambda \alpha_{1}\right) \in\{0,1\}, y\left(\lambda \alpha_{1}\right)=1 / 2$. Поэтому $x^{\prime}\left(\lambda \alpha_{1}\right) \in\{0,1\}, \rho x^{\prime}\left(\lambda \alpha_{1}\right)=1-x^{\prime}\left(\lambda \alpha_{1}\right) \in\{0,1\}, y^{\prime}\left(\lambda \alpha_{1}\right)=\tau y^{\prime}\left(\lambda \alpha_{1}\right)=$ $1 / 2$.

В ситуации (8.8.1) можно считать, что $y_{0}^{\prime}=\sigma x_{0}^{\prime}, \tau y_{0}^{\prime}=\sigma y_{0}^{\prime}$. Поэтому можно считать, что $y^{\prime}=\sigma x^{\prime}, \sigma y^{\prime}$ лежит над $\sigma y_{0}^{\prime}=\tau y_{0}^{\prime}$. Соотношение (7.4.3) влечет $1 / 2=y^{\prime}\left(\lambda \alpha_{1}^{ \pm 1}\right)=\sigma y^{\prime}\left(\sigma\left(\lambda \alpha_{1}^{ \pm 1}\right)\right)=\sigma y^{\prime}\left(\lambda \alpha_{2}^{ \pm 1}\right)$. С другой стороны, $\sigma y^{\prime}\left(\lambda \alpha_{2}^{ \pm 1}\right)=$ $1 / 2$ влечет

$$
\sigma y^{\prime}\left(\lambda \alpha_{2}+\lambda \alpha_{2}^{-1}\right) \geqslant \inf \left\{\sigma y^{\prime}\left(\lambda \alpha_{2}\right), \sigma y^{\prime}\left(\lambda \alpha_{2}^{-1}\right)\right\}=1 / 2 .
$$

Диаграмма (8.8.1) показывает, что $\sigma y^{\prime}$ лежит над $\tau y_{0}^{\prime}$. Поэтому мы получаем соотношение

$$
\tau y_{0}^{\prime}\left(\lambda \alpha_{2}+\lambda \alpha_{2}^{-1}\right) \geqslant 1 / 2
$$

Более того, $1 / 2=y^{\prime}\left(\lambda \alpha_{1}^{ \pm 1}\right)$ влечет $1 / 2=\tau y^{\prime}\left(\tau\left(\lambda \alpha_{1}^{ \pm 1}\right)\right)=\tau y^{\prime}\left(\lambda \alpha_{1}^{ \pm 1}\right)$. Поэтому получаем соотношение

$$
\tau y_{0}^{\prime}\left(\lambda \alpha_{1}+\lambda \alpha_{1}^{-1}\right) \geqslant 1 / 2 .
$$

Тогда (7.8.2) дает соотношение

$$
\begin{gathered}
\tau y_{0}^{\prime}\left(\left(\lambda \alpha_{1}+\lambda \alpha_{1}^{-1}\right)\left(\lambda \alpha_{2}+\lambda \alpha_{2}^{-1}\right)+\left(\lambda \alpha_{1}+\lambda \alpha_{1}^{-1}\right)\left(\lambda \alpha_{3}+\lambda \alpha_{3}^{-1}\right)\right. \\
\left.+\left(\lambda \alpha_{2}+\lambda \alpha_{2}^{-1}\right)\left(\lambda \alpha_{3}+\lambda \alpha_{3}^{-1}\right)\right)=0
\end{gathered}
$$

что противоречит соотношениям (8.8.3) и (8.8.4) (заметим, что $\lambda \alpha_{3}+\lambda \alpha_{3}^{-1}$-целое алгебраическое число в $F_{0}^{\prime}$, поэтому $\left.\tau y_{0}^{\prime}\left(\lambda \alpha_{3}+\lambda \alpha_{3}^{-1}\right) \geqslant 0\right)$. 
8.9. Рассмотрим случай (8.5.3). В этой ситуации $2=\left[F_{0 x_{0}^{\prime}}^{\prime}: \mathbb{Q}_{p_{v}}\right]=$ $\left[F_{0 y_{0}^{\prime}}^{\prime}: \mathbb{Q}_{p_{v}}\right]=\left[F_{0 \tau y_{0}^{\prime}}^{\prime}: \mathbb{Q}_{p_{v}}\right], F_{0 x_{0}^{\prime}}^{\prime}=F_{0}^{\prime} \otimes_{F_{0}} F_{0 x_{0}}$, поэтому

$$
\left[F_{0 x_{0}}: \mathbb{Q}_{p_{v}}\right]=1, \quad\left[F_{0 y_{0}}: \mathbb{Q}_{p_{v}}\right]=2 .
$$

Имеем $F \otimes_{\mathbb{Q}} \mathbb{Q}_{p_{v}}=F_{x} \oplus F_{y} \oplus F_{\rho y}, F_{x}=F \otimes_{F_{0}} F_{0 x_{0}}, F_{y} \oplus F_{\rho y}=F \otimes_{F_{0}} F_{0 y_{0}}$, поэтому (8.9.1) влечет $\left[F_{x}: \mathbb{Q}_{p_{v}}\right]=2,\left[F_{y}: \mathbb{Q}_{p_{v}}\right]=\left[F_{\rho y}: \mathbb{Q}_{p_{v}}\right]=2$.

В силу (7.3.1) и (7.4.1) имеем $x\left(\lambda \alpha_{1}^{ \pm 1}\right)=1 / 2, y\left(\lambda \alpha_{1}^{ \pm 1}\right) \in\{0,1 / 2,1\}$. Если $y\left(\lambda \alpha_{1}^{ \pm 1}\right)=1 / 2$, то в силу (7.4.4) $\alpha_{1}^{2}$ - корень из единицы, что противоречит мультипликативной независимости $\lambda, \alpha_{1}, \alpha_{2}, \alpha_{3}$. Поэтому $y\left(\lambda \alpha_{1}^{ \pm 1}\right) \in\{0,1\}$. Можно считать, что $y^{\prime}$ лежит над $y_{0}^{\prime}$. В силу (8.1.2) $\operatorname{Gal}\left(F^{\prime} / \mathbb{Q}\right)=\{1, \rho\} \times \operatorname{Gal}\left(F_{0}^{\prime} / \mathbb{Q}\right)$. Легко видеть, что в этом случае

$$
\operatorname{Gal}\left(F^{\prime} / F\right)=\operatorname{Gal}\left(F_{0}^{\prime} / F_{0}\right)=\{1, \tau\} .
$$

С другой строны, $\left.\tau\right|_{F}=1$ в силу (8.9.2). В частности,

$$
\tau\left(\lambda \alpha_{1}\right)=\lambda \alpha_{1} .
$$

Следовательно,

$$
\tau\left(\lambda \alpha_{2}\right)=\lambda \alpha_{3}, \quad \tau\left(\lambda \alpha_{3}\right)=\lambda \alpha_{2} .
$$

Действительно, имеем $\tau \sigma=\sigma^{2} \tau$ в $S_{3}=\operatorname{Gal}\left(F_{0}^{\prime} / \mathbb{Q}\right)$, поэтому из (8.1.2), (8.1.3), (8.9.3) следует, что

$$
\tau\left(\lambda \alpha_{2}\right)=\tau \sigma\left(\lambda \alpha_{1}\right)=\sigma^{2} \tau\left(\lambda \alpha_{1}\right)=\sigma^{2}\left(\lambda \alpha_{1}\right)=\lambda \alpha_{3} .
$$

Рассмотрим часть пополнения диаграммы (8.6.2) точками $F$ и $F^{\prime}$ :

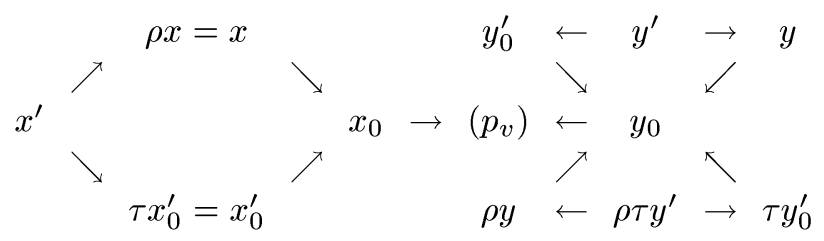

Заметим, что $y^{\prime} \neq \rho y^{\prime}$. С другой стороны, $\operatorname{Gal}\left(F^{\prime} / \mathbb{Q}\right)$ действует транзитивно на множестве $\left\{x^{\prime} \mid p_{v}\right\}$. Поэтому $x^{\prime}=\gamma y^{\prime}$ для некоторого $\gamma \in \operatorname{Gal}\left(F^{\prime} / \mathbb{Q}\right)$. В силу (7.4.5) $x^{\prime}=\gamma y^{\prime} \neq \gamma \rho y^{\prime}=\rho \gamma y^{\prime}=\rho x^{\prime}$. Поэтому получаем объединение следуюших диаграмм:

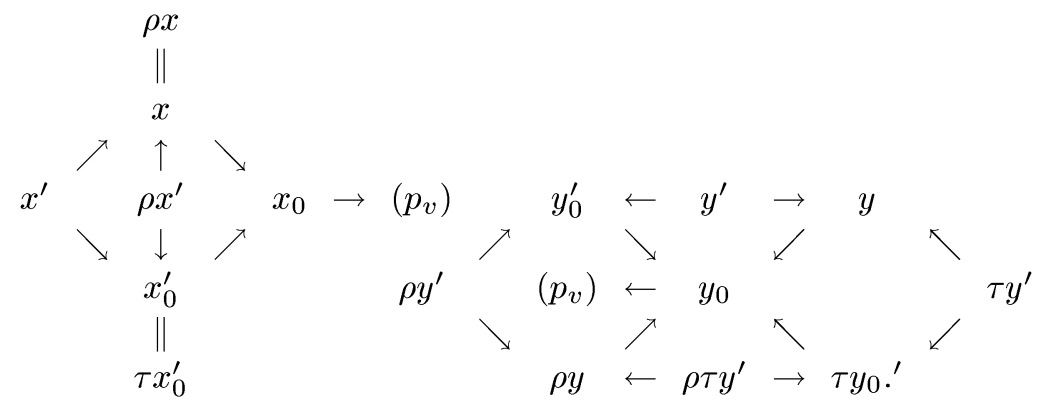


8.10. Из (8.9.2) следует, что $\left\{x^{\prime}, \tau x^{\prime}\right\}$ - множество всех точек поля $F^{\prime}$, лежаших над $x$. Из диаграммы (8.9.5) видно, что $\tau x^{\prime}=\rho x^{\prime}$. Поэтому

$$
x^{\prime}=\rho \tau x^{\prime}
$$

Вьводим из (7.2.1), (8.10.1), (8.9.3), (8.9.4) следуюшие соотношения:

$$
\begin{aligned}
& x^{\prime}\left(\lambda \alpha_{1}\right)=\rho \tau x^{\prime}\left(\lambda \alpha_{1}\right)=x^{\prime}\left(\rho \tau\left(\lambda \alpha_{1}\right)\right)=x^{\prime}\left(\rho\left(\lambda \alpha_{1}\right)\right)=x^{\prime}\left(\lambda \alpha_{1}^{-1}\right), \\
& x^{\prime}\left(\lambda \alpha_{2}\right)=\rho \tau x^{\prime}\left(\lambda \alpha_{2}\right)=x^{\prime}\left(\rho \tau\left(\lambda \alpha_{2}\right)\right)=x^{\prime}\left(\rho\left(\lambda \alpha_{3}\right)\right)=x^{\prime}\left(\lambda \alpha_{3}^{-1}\right) .
\end{aligned}
$$

Следовательно, $x^{\prime}\left(\alpha_{1}^{2}\right)=0, x^{\prime}\left(\alpha_{2} \alpha_{3}\right)=0$. Соотношение $\lambda^{2}=p_{v}$ влечет

$$
\sigma\left(\left(\alpha_{1} \alpha_{2} \alpha_{3}\right)^{2}\right)=\left(\alpha_{1} \alpha_{2} \alpha_{3}\right)^{2}, \quad \tau\left(\left(\alpha_{1} \alpha_{2} \alpha_{3}\right)^{2}\right)=\left(\alpha_{1} \alpha_{2} \alpha_{3}\right)^{2} .
$$

Поэтому

$$
\begin{aligned}
& x^{\prime}\left(\left(\alpha_{1} \alpha_{2} \alpha_{3}\right)^{2}\right)=0, \quad \sigma x^{\prime}\left(\left(\alpha_{1} \alpha_{2} \alpha_{3}\right)^{2}\right)=0, \quad \sigma^{2} x^{\prime}\left(\left(\alpha_{1} \alpha_{2} \alpha_{3}\right)^{2}\right)=0, \\
& \tau x^{\prime}\left(\left(\alpha_{1} \alpha_{2} \alpha_{3}\right)^{2}\right)=0, \quad \rho x^{\prime}\left(\left(\alpha_{1} \alpha_{2} \alpha_{3}\right)^{-2}\right)=0, \quad \rho x^{\prime}\left(\left(\alpha_{1} \alpha_{2} \alpha_{3}\right)^{2}\right)=0 .
\end{aligned}
$$

С другой стороны,

$$
\operatorname{Gal}\left(F^{\prime} / \mathbb{Q}\right) x^{\prime}=\left\{1, \sigma, \sigma^{2}, \tau, \tau \sigma, \tau \sigma^{2}, \rho, \rho \sigma \rho \sigma^{2}, \rho \tau, \rho \tau \sigma, \rho \tau \sigma^{2}\right\} x^{\prime},
$$

поэтому для любой точки $w^{\prime}$ поля $F^{\prime}$, лежашей над $p_{v}$, имеем соотношение $w^{\prime}\left(\left(\alpha_{1} \alpha_{2} \alpha_{3}\right)^{2}\right)=0$. Следовательно, для всех $w^{\prime} \mid p_{v}$

$$
\left|\left(\alpha_{1} \alpha_{2} \alpha_{3}\right)^{2}\right|_{w^{\prime}}=1
$$

Пусть $u^{\prime}$ - неархимедова точка $F^{\prime}$, не лежашая над $p_{v}$. Тогда соотношение Вейля

$$
\left(\lambda \alpha_{1} \lambda \alpha_{2} \lambda \alpha_{3}\right)^{2} \rho\left(\lambda \alpha_{1} \lambda \alpha_{2} \lambda \alpha_{3}\right)^{2}=p_{v}^{6}
$$

влечет соотношение

$$
u^{\prime}\left(\left(\lambda \alpha_{1} \lambda \alpha_{2} \lambda \alpha_{3}\right)^{2}\right)+u^{\prime}\left(\rho\left(\lambda \alpha_{1} \lambda \alpha_{2} \lambda \alpha_{3}\right)^{2}\right)=0 .
$$

Напомним, что $\lambda \alpha_{i}^{ \pm 1}$ - целые алгебраические числа. Следовательно, оба слагаемые в (8.10.4) неотрицательны. Поэтому $u^{\prime}\left(\left(\alpha_{1} \alpha_{2} \alpha_{3}\right)^{2}\right)=0$,

$$
\left|\left(\alpha_{1} \alpha_{2} \alpha_{3}\right)^{2}\right|_{u^{\prime}}=1
$$

С другой стороны, (8.10.3) влечет соотношение

$$
\left|\left(\alpha_{1} \alpha_{2} \alpha_{3}\right)^{2}\right|_{\infty}=1
$$


для любой архимедовой точки $\infty$ поля $F^{\prime}$. Напомним следующий элементарньй результат.

8.11. ЛЕмма [3, гл. $2, \S 18$, лемма 18.2]. Пусть $\alpha$ - такое алгебраическое число, что $|\alpha|=1$ для всех норм $|\cdot|: \overline{\mathbb{Q}} \rightarrow \mathbb{R}$. Тогда $\alpha-$ корень из единиць.

8.12. Мы выводим из леммы 8.11 и $(8.10 .2),(8.10 .5),(8.10 .6)$, что $\left(\alpha_{1} \alpha_{2} \alpha_{3}\right)^{2}$ - корень из единищы; это противоречит мультипликативной независимости $\lambda, \alpha_{1}$, $\alpha_{2}, \alpha_{3}$.

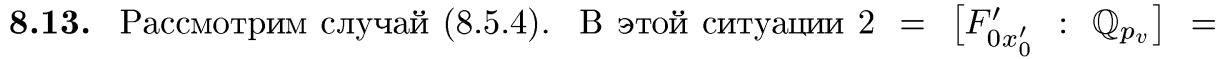
$\left[F_{0 y_{0}^{\prime}}^{\prime}: \mathbb{Q}_{p_{v}}\right]=\left[F_{0 \tau y_{0}^{\prime}}^{\prime}: \mathbb{Q}_{p_{v}}\right], F_{0 x_{0}^{\prime}}^{\prime}=F_{0}^{\prime} \otimes_{F_{0}} F_{0 x_{0}}$, поэтому

$$
\left[F_{0 x_{0}}: \mathbb{Q}_{p_{v}}\right]=1, \quad\left[F_{0 y_{0}}: \mathbb{Q}_{p_{v}}\right]=2 .
$$

Имеем $F \otimes_{\mathbb{Q}} \mathbb{Q}_{p_{v}}=F_{x} \oplus F_{\rho x} \oplus F_{y} \oplus F_{\rho y}, F_{x} \oplus F_{\rho x}=F \otimes_{F_{0}} F_{0 x_{0}}, F_{y} \oplus F_{\rho y}=F \otimes_{F_{0}}$ $F_{0 y_{0}}$, поэтому $(8.13 .1)$ влечет $\left[F_{x}: \mathbb{Q}_{p_{v}}\right]=\left[F_{\rho x}: \mathbb{Q}_{p_{v}}\right]=1,\left[F_{y}: \mathbb{Q}_{p_{v}}\right]=\left[F_{\rho y}:\right.$ $\left.\mathbb{Q}_{p_{v}}\right]=2$. В силу (7.3.1) и (7.4.1) имеем

$$
\begin{array}{ll}
x\left(\lambda \alpha_{1}\right) \in\{0,1\}, \quad \rho x\left(\lambda \alpha_{1}\right)=1-x\left(\lambda \alpha_{1}\right) \in\{0,1\}, \\
y\left(\lambda \alpha_{1}\right) \in\{0,1 / 2,1\}, \quad \rho y\left(\lambda \alpha_{1}\right)=1-y\left(\lambda \alpha_{1}\right) \in\{0,1 / 2,1\} .
\end{array}
$$

Если $y\left(\lambda \alpha_{1}\right) \in\{0,1\}$, то $J_{v}$ - обыкновенное абелево многообразие.

8.14. Далее можно считать, что $y\left(\lambda \alpha_{1}\right)=1 / 2$.

Рассмотрим часть пополнения диаграммы (8.6.2) точками поля $F^{\prime}$ :

$$
\begin{aligned}
& y_{0}^{\prime} \leftarrow y^{\prime} \\
& \downarrow \\
& \left(p_{v}\right) \leftarrow \tau y_{0}^{\prime} \leftarrow \tau y^{\prime}
\end{aligned}
$$

С другой стороны, пополнение диаграммы (8.5.4) точками поля $F^{\prime}$ дает диаграмму

$$
\begin{aligned}
& x^{\prime} \rightarrow x \quad y \leftarrow y^{\prime} \\
& \rho x^{\prime} \rightarrow \rho x \rightarrow x_{0} \rightarrow\left(p_{v}\right) \leftarrow y_{0} \leftarrow \rho y \leftarrow \rho y^{\prime}
\end{aligned}
$$

Выводим из (7.4.2) и (8.14.2), что

$$
x^{\prime}\left(\lambda \alpha_{1}^{ \pm 1}\right) \in\{0,1\}, \quad \rho x^{\prime}\left(\lambda \alpha_{1}^{ \pm 1}\right) \in\{0,1\}, \quad y^{\prime}\left(\lambda \alpha_{1}^{ \pm 1}\right)=1 / 2 .
$$

В ситуации (8.14.1) можно считать, что $y_{0}^{\prime}=\sigma x_{0}^{\prime}, \tau y_{0}^{\prime}=\sigma y_{0}^{\prime}$. Поэтому можно считать, что $y^{\prime}=\sigma x^{\prime}, \sigma y^{\prime}$ лежит над $\tau y_{0}^{\prime}$. Соотношение (7.4.3) влечет $1 / 2=$ $y^{\prime}\left(\lambda \alpha_{1}^{ \pm 1}\right)=\sigma y^{\prime}\left(\sigma\left(\lambda \alpha_{1}^{ \pm 1}\right)\right)=\sigma y^{\prime}\left(\lambda \alpha_{2}^{ \pm 1}\right)$. Поэтому

$$
\sigma y^{\prime}\left(\lambda \alpha_{2}+\lambda \alpha_{2}^{-1}\right) \geqslant \inf \left\{\sigma y^{\prime}\left(\lambda \alpha_{2}\right), \sigma y^{\prime}\left(\lambda \alpha_{2}^{-1}\right)\right\}=1 / 2
$$

С другой стороны, $\sigma y^{\prime}$ лежит над $\tau y_{0}^{\prime}$. Поэтому получаем

$$
\tau y_{0}^{\prime}\left(\lambda \alpha_{2}+\lambda \alpha_{2}^{-1}\right) \geqslant 1 / 2
$$


Далее, $1 / 2=y^{\prime}\left(\lambda \alpha_{1}^{ \pm 1}\right)$ влечет $1 / 2=\tau y^{\prime}\left(\tau\left(\lambda \alpha_{1}^{ \pm 1}\right)\right)=\tau y^{\prime}\left(\lambda \alpha_{1}^{ \pm 1}\right)$. Из (8.14.1) следует, что $\tau y^{\prime}$ лежит над $\tau y_{0}^{\prime}$, поэтому получаем соотношение

$$
\tau y_{0}^{\prime}\left(\lambda \alpha_{1}+\lambda \alpha_{1}^{-1}\right) \geqslant 1 / 2
$$

Ясно, что (8.14.3) и (8.14.4) противоречат (7.8.2).

8.15. Предположим, что $x_{0}, y_{0}, z_{0}$ - все точки кубического поля $F_{0}$, лежашие над $p_{v}$. Можно считать, что имеются следуюшие варианты:

$$
\begin{aligned}
& y_{0} \leftarrow y=\rho y \\
& x=\rho x \rightarrow x_{0} \rightarrow \begin{array}{c}
\downarrow \\
\left(p_{v}\right)
\end{array} \leftarrow z_{0} \quad \leftarrow z=\rho z ;
\end{aligned}
$$

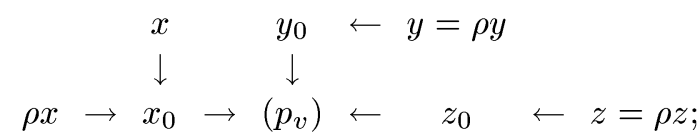

$$
\begin{aligned}
& \begin{array}{ccc}
y & \rightarrow & y_{0} \\
& \downarrow & \leftarrow
\end{array} \\
& x=\rho x \rightarrow x_{0} \rightarrow\left(p_{v}\right) \\
& z \rightarrow z_{0} \quad \leftarrow \rho z ;
\end{aligned}
$$

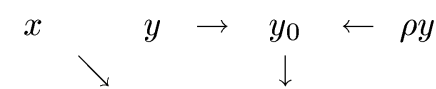

$$
\begin{aligned}
& x_{0} \rightarrow\left(p_{v}\right) \\
& \rho{ }^{\nearrow} \quad z \rightarrow z_{0} \leftarrow \rho z
\end{aligned}
$$

8.16. Рассмотрим случай (8.15.1). В силу леммы 7.4 имеем $x\left(\lambda \alpha_{1}^{ \pm 1}\right)=$ $y\left(\lambda \alpha_{1}^{ \pm 1}\right)=z\left(\lambda \alpha_{1}^{ \pm 1}\right)=1 / 2$, поэтому $\alpha_{1}^{2}$ - корень из единищы, что противоречит мультипликативной независимости $\lambda, \alpha_{1}, \alpha_{2}, \alpha_{3}$.

8.17. Рассмотрим случай (8.15.2). Имеем $F \otimes_{\mathbb{Q}} \mathbb{Q}_{p_{v}}=F_{x} \oplus F_{\rho x} \oplus F_{y} \oplus F_{z}$, $\left[F_{y}: \mathbb{Q}_{p_{v}}\right]$ и $\left[F_{z}: \mathbb{Q}_{p_{v}}\right]$ - четные числа в силу соотношений $y=\rho y, z=\rho z[15]$, поэтому $\left[F_{x}: \mathbb{Q}_{p_{v}}\right]=\left[F_{\rho x}: \mathbb{Q}_{p_{v}}\right]=1,\left[F_{y}: \mathbb{Q}_{p_{v}}\right]=\left[F_{z}: \mathbb{Q}_{p_{v}}\right]=2$. Следовательно,

$x\left(\lambda \alpha_{1}\right) \in\{0,1\}, \quad \rho x\left(\lambda \alpha_{1}\right)=1-x\left(\lambda \alpha_{1}\right) \in\{0,1\}, \quad y\left(\lambda \alpha_{1}^{ \pm 1}\right)=z\left(\lambda \alpha_{1}^{ \pm 1}\right)=1 / 2$.

8.18. Предположим, что $F_{0} / \mathbb{Q}$ - нормальное расширение с группой Галуа $\left\{1, \sigma, \sigma^{2}\right\}$. Рассмотрим часть пополнения диаграммы (8.15.2) точками $F^{\prime}$ :

$$
\begin{aligned}
& x^{\prime} \rightarrow x \quad y_{0} \leftarrow y=\rho y \leftarrow y^{\prime} \\
& \rho x^{\prime} \rightarrow \rho x \rightarrow x_{0} \rightarrow\left(p_{v}\right) \leftarrow z_{0} \quad \leftarrow z=\rho z \leftarrow z^{\prime}
\end{aligned}
$$

Можно считать, что $z_{0}=\sigma y_{0}$ и $z^{\prime}=\sigma y^{\prime}$. 
Из соотношений (7.4.2), (7.4.3) следует, что

$$
1 / 2=y^{\prime}\left(\lambda \alpha_{1}^{ \pm 1}\right)=z^{\prime}\left(\lambda \alpha_{1}^{ \pm 1}\right)=\sigma y^{\prime}\left(\sigma\left(\lambda \alpha_{1}^{ \pm 1}\right)\right)=z^{\prime}\left(\lambda \alpha_{2}^{ \pm 1}\right) .
$$

Поэтому

$$
\begin{gathered}
z_{0}\left(\lambda \alpha_{1}+\lambda \alpha_{1}^{-1}\right) \geqslant \inf \left\{z^{\prime}\left(\lambda \alpha_{1}\right), z^{\prime}\left(\lambda \alpha_{1}^{-1}\right)\right\}=1 / 2 \\
z_{0}\left(\lambda \alpha_{2}+\lambda \alpha_{2}^{-1}\right) \geqslant 1 / 2
\end{gathered}
$$

что противоречит (7.8.2).

8.19. Предположим, что $F_{0}^{\prime} \neq F_{0}$. В этом случае $\operatorname{Gal}\left(F_{0}^{\prime} / F_{0}\right)=\{1, \tau\}$, где $\tau$ - элемент порядка 2 в $S_{3}=\operatorname{Gal}\left(F_{0}^{\prime} / \mathbb{Q}\right)$. Очевидно, $\operatorname{Card}\left(\left\{w_{0}^{\prime} \mid p_{v}\right\}\right) \in\{3,6\}$. Поэтому пополнение диаграммы

$$
\begin{gathered}
y_{0} \\
\downarrow \\
x_{0} \rightarrow\left(p_{v}\right) \leftarrow z_{0}
\end{gathered}
$$

точками $F_{0}^{\prime}$ является одним из следующих вариантов:

$$
\begin{aligned}
y_{0} & \leftarrow y_{0}^{\prime} \\
\downarrow & \\
x_{0}^{\prime} \rightarrow x_{0} \rightarrow\left(p_{v}\right) & \leftarrow z_{0} \leftarrow z_{0}^{\prime}
\end{aligned}
$$

и

$$
\begin{aligned}
& x_{0}^{\prime} \backslash y_{0}^{\prime} \rightarrow \begin{array}{c}
y_{0} \\
\downarrow
\end{array} \\
& x_{0} \rightarrow\left(p_{v}\right) \\
& \tau x_{0}^{\prime} \quad z_{0}^{\prime} \rightarrow z_{0} \leftarrow \tau z_{0}^{\prime}
\end{aligned}
$$

В любом случае можно считать, что $z_{0}^{\prime}=\sigma y_{0}^{\prime}$.

Имеем

$$
\begin{aligned}
& z_{0}^{\prime}\left(\lambda \alpha_{1}+\lambda \alpha_{1}^{-1}\right) \geqslant \inf \left\{z^{\prime}\left(\lambda \alpha_{1}\right), z^{\prime}\left(\lambda \alpha_{1}^{-1}\right)\right\}=1 / 2 \\
& y_{0}^{\prime}\left(\lambda \alpha_{1}+\lambda \alpha_{1}^{-1}\right) \geqslant \inf \left\{y^{\prime}\left(\lambda \alpha_{1}\right), y^{\prime}\left(\lambda \alpha_{1}^{-1}\right)\right\}=1 / 2
\end{aligned}
$$

поэтому

$$
z_{0}^{\prime}\left(\lambda \alpha_{2}+\lambda \alpha_{2}^{-1}\right)=\sigma y_{0}^{\prime}\left(\sigma\left(\lambda \alpha_{1}+\lambda \alpha_{1}^{-1}\right)\right)=y_{0}^{\prime}\left(\lambda \alpha_{1}+\lambda \alpha_{1}^{-1}\right) \geqslant 1 / 2,
$$

что противоречит (7.8.2).

8.20. Рассмотрим случай (8.15.3). В этой ситуации

$x\left(\lambda \alpha_{1}\right)=1 / 2, \quad y\left(\lambda \alpha_{1}\right)=1-\rho y\left(\lambda \alpha_{1}\right) \in\{0,1\}, \quad z\left(\lambda \alpha_{1}\right)=1-\rho z\left(\lambda \alpha_{1}\right) \in\{0,1\}$. 
8.21. Предположим, что $F_{0}^{\prime}=F_{0}$. В этом случае $F=F^{\prime}$, поэтому $F / \mathbb{Q}$ нормальное расширение, и в точности пять точек, $x=\rho x, y, \rho y, z, \rho z$, сопряжены друг другу, что противоречит соотношению $6=\operatorname{Card}(\operatorname{Gal}(F / \mathbb{Q}))$.

8.22. Предположим, что $F_{0}^{\prime} \neq F_{0}$. Тогда пополнение диаграммы

$$
\begin{gathered}
y_{0} \\
\downarrow \\
x_{0} \rightarrow\left(p_{v}\right) \leftarrow z_{0}
\end{gathered}
$$

точками $F_{0}^{\prime}$ является одним из следуюших вариантов:

$$
\begin{aligned}
y_{0} & \leftarrow y_{0}^{\prime}=\tau y_{0}^{\prime} \\
\downarrow & \\
x_{0}^{\prime}=\tau x_{0}^{\prime} \rightarrow x_{0} \rightarrow\left(p_{v}\right) & \leftarrow z_{0} \quad \leftarrow z_{0}^{\prime}=\tau z_{0}^{\prime}
\end{aligned}
$$

и

$$
\begin{aligned}
& x_{0}^{\prime} \backslash \begin{array}{c}
y_{0}^{\prime} \rightarrow \quad y_{0} \\
\downarrow
\end{array} \\
& x_{0} \rightarrow\left(p_{v}\right) \\
& \tau x_{0}^{\prime} \quad z_{0}^{\prime} \rightarrow z_{0} \leftarrow \tau z_{0}^{\prime}
\end{aligned}
$$

потому что все точки $w_{0}^{\prime} \mid\left(p_{v}\right)$ сопряжены относительно действия $\operatorname{Gal}\left(F_{0}^{\prime} / F_{0}\right)=$ $S_{3}$, и число таких точек равно 3 или 6.

8.23. Рассмотрим случай (8.22.1). Мы имеем $x^{\prime} \mid x \Rightarrow x^{\prime}\left(\lambda \alpha_{1}^{ \pm 1}\right)=1 / 2$. Поэтому $x_{0}^{\prime}\left(\lambda \alpha_{1}+\lambda \alpha_{1}^{-1}\right) \geqslant 1 / 2$. Можно считать, что $y_{0}^{\prime}=\sigma x_{0}^{\prime}$. Поэтому $\sigma x_{0}^{\prime}\left(\sigma\left(\lambda \alpha_{1}+\lambda \alpha_{1}^{-1}\right)\right) \geqslant 1 / 2$ и

$$
y_{0}^{\prime}\left(\lambda \alpha_{2}+\lambda \alpha_{2}^{-1}\right) \geqslant 1 / 2 .
$$

С другой строны, $\tau y_{0}^{\prime}=y_{0}^{\prime}$, поэтому (8.23.1) влечет

$$
1 / 2 \leqslant \tau y_{0}^{\prime}\left(\tau\left(\lambda \alpha_{2}+\lambda \alpha_{2}^{-1}\right)\right)=y_{0}^{\prime}\left(\lambda \alpha_{3}+\lambda \alpha_{3}^{-1}\right),
$$

что противоречит (7.8.2).

8.24. Рассмотрим случай (8.22.2). Точки $w^{\prime} \mid p_{v}$ сопряжены друг другу относительно действия $\operatorname{Gal}\left(F^{\prime} / \mathbb{Q}\right)=\{1, \rho\} \times S_{3}$. В силу $(8.9 .2)$

$$
\operatorname{Gal}\left(F^{\prime} / F\right)=\operatorname{Gal}\left(F_{0}^{\prime} / F_{0}\right)=\{1, \tau\} .
$$

Поэтому $\left\{x^{\prime}, \tau x^{\prime}\right\}$ - множество всех точек поля $F^{\prime}$, лежаших над $x=\rho x$. С другой стороны, $y^{\prime} \neq \rho y^{\prime}$ (потому что $y^{\prime} \mid y$ и $\left.\rho y^{\prime} \mid \rho y(y \neq \rho y)\right)$ и $x^{\prime}=\gamma y^{\prime}$ для некоторого $\gamma \in \operatorname{Gal}\left(F^{\prime} / \mathbb{Q}\right)$. Поэтому $x^{\prime}=\gamma y^{\prime} \neq \gamma \rho y^{\prime}=\rho \gamma y^{\prime}=\rho x^{\prime}$. Следовательно, $\tau x^{\prime}=\rho x^{\prime}, x^{\prime}=\rho \tau x^{\prime}$. Мы получаем соотношение (8.10.1) в новой ситуации. Поэтому мы можем исключить этот вариант с помощью процедуры пп. 8.10-8.12.

8.25. Рассмотрим случай (8.15.4). Имеем

$\left[F_{x}: \mathbb{Q}_{p_{v}}\right]=\left[F_{\rho x}: \mathbb{Q}_{p_{v}}\right]=\left[F_{y}: \mathbb{Q}_{p_{v}}\right]=\left[F_{\rho y}: \mathbb{Q}_{p_{v}}\right]=\left[F_{z}: \mathbb{Q}_{p_{v}}\right]=\left[F_{\rho z}: \mathbb{Q}_{p_{v}}\right]=1$, поэтому для любого $w \mid p_{v} w\left(\lambda \alpha_{1}\right) \in\{0,1\}$. Следовательно, $J_{v}$ - обыкновенное абелево многообразие. 


\section{§9. Случай приводимого характеристического полинома}

9.1. Предположим, что характеристический полином $P(t)=\mathrm{Pc}_{J}\left(\rho_{l}\left(F_{\bar{v}}^{-1}\right), t\right)$ приводим над полем $\mathbb{Q}$. Из соотношения (7.2.1) следует, что $\mathrm{Pc}_{J}\left(\rho_{l}\left(F_{\bar{v}}^{-1}\right), t\right)-$ произведение трех неприводимых полиномов над $\mathbb{Q}$ или $\mathrm{Pc}_{J}\left(\rho_{l}\left(F_{\bar{v}}^{-1}\right), t\right)=P(t) \cdot Q(t)$, где $P(t), Q(t)$ неприводимы над $\mathbb{Q}, \operatorname{deg} P(t)=4, \operatorname{deg} Q(t)=2$.

9.2. Если $\mathrm{Pc}_{J}\left(\rho_{l}\left(F \bar{v}^{-1}\right), t\right)$ - произведение трех неприводимых полиномов над $\mathbb{Q}$, то $J_{v}$ изогенно произведению трех обыкновенных эллиптических кривых. Это следует из теории Хонды-Тэйта [32], [33, лемма 1.0.3].

9.3. Предположим, что $\mathrm{Pc}_{J}\left(\rho_{l}\left(F_{\bar{v}}^{-1}\right), t\right)=P(t) \cdot Q(t)$. Можно считать, что $\lambda \alpha_{3}^{ \pm 1}$ - корень $Q(t), \lambda \alpha_{1}^{ \pm 1}, \lambda \alpha_{2}^{ \pm 1}-$ корни $P(t)$. В этом случае $J_{v}$ изогенно произведению $A \times E$, где $A$ определено в п. 7.3, и $E$ - обыкновенная эллиптическая кривая, $\operatorname{End}^{0}(A)=F=\mathbb{Q}\left(\lambda \alpha_{1}\right), \operatorname{End}^{0}(E)=\mathbb{Q}\left(\lambda \alpha_{3}\right)$ [33, лемма 1.0.3].

Из неприводимости $P(t)$ и леммы 7.2 следует, что $[F: \mathbb{Q}]=4$.

9.4. Предположим, что $x_{0}$ - единственная точка квадратичного поля $F_{0}$, лежащая над $p_{v}$. Мы имеем два варианта пополнения диаграммы $x_{0} \rightarrow\left(p_{v}\right)$ точками поля $F$ :

$$
x=\rho x \rightarrow x_{0} \rightarrow\left(p_{v}\right)
$$

И

$$
\begin{aligned}
& \\
& \\
& \\
\rho x \rightarrow x_{0} & \rightarrow\left(p_{v}\right)
\end{aligned}
$$

9.5. Рассмотрим случай (9.4.1). В силу леммы $7.4 x\left(\lambda \alpha_{1}^{ \pm 1}\right)=1 / 2$, поэтому $\alpha_{1}^{2}$ - корень из единицы, что противоречит мультипликативной независимости $\lambda$, $\alpha_{1}, \alpha_{2}, \alpha_{3}$.

9.6. Рассмотрим случай (9.4.2). Мы имеем $\left[F_{x}: \mathbb{Q}_{p_{v}}\right]=\left[F_{\rho x}: \mathbb{Q}_{p_{v}}\right]=2$. В силу (7.3.1) $x\left(\lambda \alpha_{1}^{ \pm 1}\right) \in\{0,1 / 2,1\}$. Предположим, что $x\left(\lambda \alpha_{1}\right)=1 / 2$. Тогда $x\left(\lambda \alpha_{1}^{ \pm 1}\right)=1 / 2, \rho x\left(\lambda \alpha_{1}^{ \pm 1}\right)=1 / 2$ и $\alpha_{1}^{2}$-корень из единицы, что противоречит мультипликативной независимости $\lambda, \alpha_{1}, \alpha_{2}, \alpha_{3}$. Следовательно, $x\left(\lambda \alpha_{1}^{ \pm 1}\right) \in\{0,1\}$, $\rho x\left(\lambda \alpha_{1}^{ \pm 1}\right) \in\{0,1\}$ и $J_{v}$ - обыкновенное абелево многообразие.

9.7. Предположим, что $x_{0}, y_{0}$ - различные точки поля $F_{0}$, лежащие над $p_{v}$. Мы имеем следуюшие варианты:

$$
\begin{gathered}
x=\rho x \rightarrow x_{0} \rightarrow\left(p_{v}\right) \leftarrow y_{0} \leftarrow y=\rho y ; \\
x \\
\downarrow \\
\rho x \rightarrow x_{0} \rightarrow\left(p_{v}\right) \leftarrow y_{0} \leftarrow y=\rho y ; \\
x \quad c \\
\downarrow \\
\rho x \rightarrow x_{0} \rightarrow\left(p_{v}\right) \leftarrow y_{0} \leftarrow \rho y
\end{gathered}
$$

9.8. Рассмотрим случай (9.7.1). В силу леммы 7.4 имеем $x\left(\lambda \alpha_{1}^{ \pm 1}\right)=1 / 2=$ $y\left(\lambda \alpha_{1}^{ \pm 1}\right)$, поэтому $\alpha_{1}^{2}$ - корень из единищы, что противоречит мультипликативной независимости $\lambda, \alpha_{1}, \alpha_{2}, \alpha_{3}$. 
9.9. Рассмотрим случай (9.7.2). Из неприводимости $P(t), Q(t)$ и леммы 7.2 следует, что $[F: \mathbb{Q}]=4$, и отображение редукции $K=\operatorname{End}^{0}(J) \rightarrow \operatorname{End}^{0}\left(J_{v}\right)=F \oplus$ $\mathbb{Q}\left(\lambda \alpha_{3}\right)$ дает вложения $K=\operatorname{End}^{0}(J) \subset F, K=\operatorname{End}^{0}(J) \subset \mathbb{Q}\left(\lambda \alpha_{3}\right)$. Легко видеть, что $F=K\left(F_{0}\right)$. Мы имеем $K \cap F_{0}=\mathbb{Q}$, потому что $F_{0}$-вполне вещественное поле. Следовательно, $K$ и $F_{0}$ линейно разделены над $\mathbb{Q}, K\left(F_{0}\right)$ - нормальное расширение $\mathbb{Q}$ степени $4[3$, гл. $5, \S 10$, п. 4 , следствие 2$]$. С другой стороны, из соотношения $F^{\prime}=F$ следует, что все точки $w \mid p_{v}$ сопряжены относительно действия $\operatorname{Gal}(F / \mathbb{Q})$, поэтому число таких точек равно 2 или 4 , что противоречит (9.7.2).

9.10. Рассмотрим случай (9.7.3). Имеем $\left[\begin{array}{lll}F_{x} & : & \mathbb{Q}_{p_{v}}\end{array}\right]=\left[\begin{array}{lll}F_{\rho x} & : & \mathbb{Q}_{p_{v}}\end{array}\right]=$ $\left[F_{y}: \mathbb{Q}_{p_{v}}\right]=\left[F_{\rho y}: \mathbb{Q}_{p_{v}}\right]=1$, поэтому для любого $w \mid p_{v} w\left(\lambda \alpha_{1}\right) \in\{0,1\}$. Следовательно, $J_{v}$ - обыкновенное абелево многообразие. Теорема 0.12 доказана.

\section{Список литературы}

1. André $Y$. On the Shafarevich and Tate conjectures for hyperkähler varieties // Math. Ann. 1996. V. 305. P. 205-248.

2. Bogomolov F. A. Sur l'algebricité des représentations l-adiques // C. R. Acad. Sc. Paris. Ser. I Math. 1980. V. 290. P. 701-703.

3. Бурбаки Н. Алгебра. Гл. 4-6. М.: Наука, 1965.

4. Бурбаки Н. Группы и алгебры Ли. М.: Мир; гл. 1-3 (1976), гл. 4-6 (1972), гл. 7-8 (1978).

5. Algebraic number theory (Proc. International Conf., Brighton, 1965) / Eds. J. W. S. Cassels, A. Frohlich. Academic Press, London, and Thompson, Washington, D.C., 1967.

6. Chi W. On the $l$-adic representations attached to simple abelian varieties of type IV // Bull. Austral. Math. Soc. 1991. V. 44. P. 71-78.

7. $C h i W$. $l$-adic and $\lambda$-adic representations associated to abelian varieties defined over number fields // Amer. J. Math. 1992. V. 114. P. 315-353.

8. Deligne P. Variétés abéliennes ordinaires sur un corps fini // Invent. Math. 1969. V. 8. P. 238-243.

9. Deligne $P$. La conjecture de Weil pour les surfaces $K 3 / /$ Invent. Math. 1972. V. 15. P. 206-226.

10. Faltings G. Endlichkeitssätze für abelsche Varietäten über Zahlkörpern // Invent. Math. 1983. V. 73. P. 349-366.

11. Mumford D. Families of abelian varieties // Algebraic Groups and Discontinuous Subgroups, Proc. Sympos. Pure Math., Amer. Math. Soc., Providence, RI, 1966. V. 9. P. 347-352.

12. Mumford D. Lectures on curves on an algebraic surface. Princeton - N. J.: Princeton Univ. Press, 1966.

13. Mumford D. (in collaboration with C.P.Ramanujam) Abelian varieties. London: Tata Inst. Fund. Res., Bombay, and Oxford Univ. Press, 1970.

14. Noot $R$. Abelian varieties - Galois representation and properties of ordinary reduction // Compositio Mathematica. 1995. V. 97. P. 161-171.

15. Ogus A. Hodge cycles and crystalline cohomology. Hodge cycles, motives, and Shimura varieties // Lecture Notes in Math. V. 900. Berlin: Springer-Verlag, 1982. P. 357-414.

16. Pink R. l-adic algebraic monodromy groups, cocharacters, and the Mumford-Tate conjecture // Preprint Universität Mannheim 11/1997.

17. Sen S. Lie algebras of Galois groups arising from Hodge-Tate modules // Ann. Math. 1973. V. 97. № 2. P. 160-170.

18. Serre J.-P. Groupes algébriques associés aux modules de Hodge-Tate // Astérisque 1979. V. 65. P. $155-188$.

19. Serre J.-P. Propriétés conjecturales des groupes de Galois motiviques et des représentations $l$-adiques // Proc. of Symposia in Pure Math. 1994. V. 55, Part 1. P. 377-400. 
20. Shimura G. Introduction to the arithmetic theory of automorphic functions. Iwanami Shoten, Publishers and Princeton University Press, 1971.

21. Танкеев С. Г. Циклы на простых абелевых многообразиях простой размерности // Изв. АН СССР. Сер. матем. 1982. Т. 46. № 1. С. 155-170.

22. Танкеев C. Г. Циклы на простых абелевых многообразиях простой размерности над числовыми полями // Изв. АН СССР. Сер. матем. 1987. Т. 51. № 6. С. 1214-1227.

23. Танкеев $C$. Г. Поверхности типа $K 3$ над числовыми полями и $l$-адические представления // Изв. АН СССР. Сер. матем. 1988. Т. 52. №6. С. 1252-1271.

24. Танкеев C. Г. Абелевы многообразия Куги-Сатаке и $l$-адические представления // Изв. РАН. Сер. матем. 1991. Т. 55. № 4. С. 877-889.

25. Танкеев C.Г. Поверхности типа $K 3$ над числовыми полями и гипотеза Мамфорда-Тэйта. II // Изв. РАН. Сер. матем. 1995. Т. 59. № 3. С. 179-206.

26. Танкеев С.Г. О следах Фробениуса // Изв. РАН. Сер. матем. 1998. Т. 62. №1. C. $165-200$.

27. Tankeev S. G. Frobenius traces and minuscule weights. Preprint of the Warwick University $39 / 1996$.

28. Tankeev S. G. Frobenius traces and minuscule weights. II. Preprint of the Institut des Hautes Études Scientifiques, Bures-sur-Yvette: IHES/M/97/20.

29. Tankeev S. G. On the existence of ordinary reductions of a simple abelian threefold of type IV. Preprint of the Institut des Hautes Études Scientifiques (Bures-sur-Yvette) IH$\mathrm{ES} / \mathrm{M} / 97 / 21$.

30. Tankeev S. G. On the existence of ordinary reductions of a $K 3$ surface over a number field. Preprint of the Institut des Hautes Études Scientifiques (Bures-sur-Yvette) IH$\mathrm{ES} / \mathrm{M} / 97 / 27$.

31. Tankeev S. G. On weights of $l$-adic representation. Preprint of the Mathematical Sciences Research Institute. Berkeley, 1997-047.

32. Tate $J$. Classes d' isogénie des variétés abéliennes sur un corps fini (d'après T. Honda). Séminaire Bourbaki 1968/69, Exposé 352 // Lecture Notes in Math. V. 179. Berlin: Springer-Verlag, 1971. P. 95-110.

33. Зархин Ю.Г. Абелевы многообразия, $l$-адические представления и $\mathrm{SL}_{2} / /$ Изв. АН CССР. Сер. матем. 1979. Т. 43. № 2. С. 294-308.

34. Zarhin Yu. G. Hodge group of K3 surface // J. für die reine und angew. Math. 1983. V. 341. P. $193-220$.

35. Зархин Ю. Г. Веса простых алгебр Ли в когомологиях алгебраических многообразий // Изв. АН СССР. Сер. матем. 1984. Т. 48. № 2. С. 264-304.

Владимирский государственный университет, ул. Горького, 87, Владимир, 600026, Россия.

Поступило в редакцию

E-mail: tankeev-rtf@vpti.vladimir.su

20.VII.1997 